\title{
Multiscale nuclear magnetic relaxation dispersion of complex liquids in bulk and confinement
}

\author{
Jean-Pierre Korb* \\ Laboratoire de Physique de la Matière Condensée, Ecole Polytechnique, CNRS, Université de Paris Saclay, 91128 Palaiseau Cedex, France \\ Sorbonne Universités, UPMC Univ. Paris 06, CNRS, PHENIX Laboratory, F-75005 Paris, France
}

Edited by J.W. Emsley and D. Neuhaus

Keywords:

Nuclear magnetic relaxation dispersion Porous media

Cements

Complex liquids

Shale oils

Proteins

\begin{abstract}
A B S T R A C T
The nuclear magnetic relaxation dispersion (NMRD) technique consists of measurement of the magneticfield dependence of the longitudinal nuclear-spin-lattice relaxation rate $1 / T_{1}$. Usually, the acquisition of the NMRD profiles is made using a fast field cycling (FFC) NMR technique that varies the magnetic field and explores a very large range of Larmor frequencies $\left(10 \mathrm{kHz}<\omega_{0} /(2 \pi)<40 \mathrm{MHz}\right.$ ). This allows extensive explorations of the fluctuations to which nuclear spin relaxation is sensitive. The FFC technique thus offers opportunities on multiple scales of both time and distance for characterizing the molecular dynamics and transport properties of complex liquids in bulk or embedded in confined environments. This review presents the principles, theories and applications of NMRD for characterizing fundamental properties such as surface correlation times, diffusion coefficients and dynamical surface affinity (NMR wettability) for various confined liquids. The basic longitudinal and transverse relaxation equations are outlined for bulk liquids. The nuclear relaxation of a liquid confined in pores is considered in detail in order to find the biphasic fast exchange relations for a liquid at proximity of a solid surface. The physical-chemistry of liquids at solid surfaces induces striking differences between NMRD profiles of aprotic and protic (water) liquids embedded in calibrated porous disordered materials. A particular emphasis of this review concerns the extension of FFC NMR relaxation to industrial applications. For instance, it is shown that the FFC technique is sufficiently rapid for following the progressive setting of cement-based materials (plasters, cement pastes, concretes). The technique also allows studies of the dynamics of hydrocarbons in proximity of asphaltene nano-aggregates and macro-aggregates in heavy crude oils as a function of the concentration of asphaltenes. It also gives new information on the wettability of petroleum fluids (brine and oil) embedded in shale oil rocks. It is useful for understanding the relations and correlations between NMR relaxation times $T_{1}$ and $T_{2}$, diffusion coefficients $D$, and viscosity $\eta$ of heavy crude oils. This is of particular importance for interpreting $T_{1}, T_{2}, 2 D T_{1}-T_{2}$ and $D-T_{2}$ correlation spectra that could be obtained down-hole, thus giving a valuable tool for investigating in situ the molecular dynamics of petroleum fluids. Another domain of interest concerns biological applications. This is of particular importance for studying the complex dynamical spectrum of a folded polymeric structure that may span many decades in frequency or time. A direct NMRD characterization of water diffusional dynamics is presented at the protein interface. NMR experiments using a shuttle technique give results well above the frequency range accessible via the FFC technique; examples of this show protein dynamics over a range from fast and localized motions to slow and delocalized collective motions involving the whole protein. This review ends by an interpretation of the origin of the proton magnetic field dependence of $T_{1}$ for different biological tissues of animals; this includes a proposal for interpreting in vivo MRI data from human brain at variable magnetic fields, where the FFC relaxation analysis suggests that brain white-matter is distinct from grey-matter, in agreement with diffusion-weighted MRI imaging.
\end{abstract}

\footnotetext{
* Address: Laboratoire de Physique de la Matière Condensée, Ecole Polytechnique, CNRS, Université de Paris Saclay, 91128 Palaiseau Cedex, France.

E-mail address: jean-pierre.korb@polytechnique.fr
} 


\section{Introduction}

This article reviews the principles and applications of multiscale nuclear magnetic relaxation dispersion (NMRD) techniques [1-4] for characterizing molecular dynamics and transport properties of complex liquids in bulk and in confinement [5,6]. Fig. 1 shows a schematic representation of the commonly used multiscale (time/distance) NMR techniques. Compared to the limited range explored by the standard relaxation technique employing a single, fixed magnetic field $\boldsymbol{B}_{0}$, the NMRD method can report on molecular dynamics from molecular to sub-micron sizes. The NMRD experiment works by measuring the values of the longitudinal relaxation time $T_{1}$ over a wide range of magnetic field strengths $B_{0}$. For protonated liquids, nuclear spin relaxation is a stimulated (not spontaneous) process induced by the coupling between the nuclear spins $I=1 / 2$ and the incoherent magnetic noise created by the molecular dynamics of the spin-bearing molecules and their surroundings. The main interest of the NMRD technique lies in decreasing the Larmor frequency $\omega_{0} / 2 \pi$ to explore correspondingly longer correlation times $\tau_{c}$ of the dipolar fluctuations that are induced by molecular dynamics and cause the spin-lattice relaxation. This relaxation is at its most efficient around $\omega_{0} \tau_{c}=1$ : the available power of the random $r f$ field is spread over a range of frequencies that extends up to about $2 \pi / \tau_{c}$, so when $\omega_{0} \tau_{c} \ll 1$ that power is spread too thinly for efficient relaxation, while for $\omega_{0} \tau_{c} \gg 1$ the motion is too slow to generate much $r f$ power at the frequency $\omega_{0}$. As a result, decreasing the magnetic field $B_{0}=\omega_{0} / \gamma$ causes the length of diffusion $\ell_{D}\left(\omega_{0}\right)=\sqrt{6 D \tau_{c}} \approx$ $\sqrt{6 D / \omega_{0}} \rightarrow d_{\text {pore }}$ to become sufficiently extended that translational diffusion of an embedded liquid in porous media can reach the average pore size $d_{\text {pore }}$. The NMRD technique thus becomes a very useful tool for studying the molecular dynamics at pore surfaces even for a fully saturated pore system.

The NMRD data described in the papers reviewed here were recorded using the typical fast-field-cycling FFC NMR sequences (see Section 3 of Ref. [5]) used on a commercially available spectrometer from Stelar s.r.l., Mede, Italy. This instrument was really a breakthrough in the field because the experimenters have to make their own instruments before. Though this commercial instrument does not have a sufficient homogeneous magnetic field for getting high resolution NMR spectra, it is designed to obtain a longitudinal nuclear magnetic relaxation profile. Fig. 2 displays 


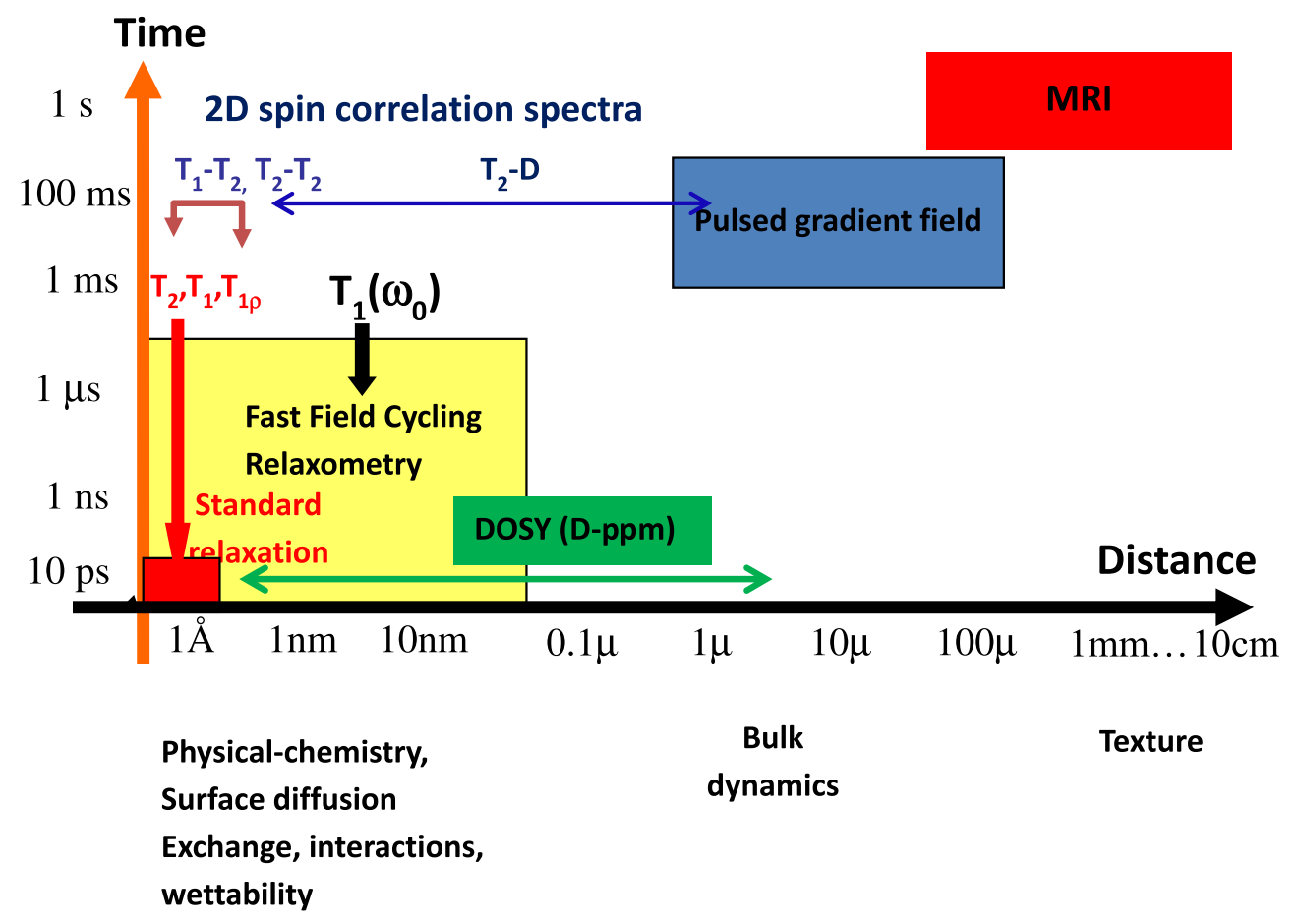

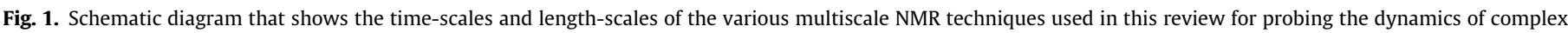
liquids in bulk and confinement. The NMRD technique can monitor liquid dynamics from molecular to sub-micron sizes.

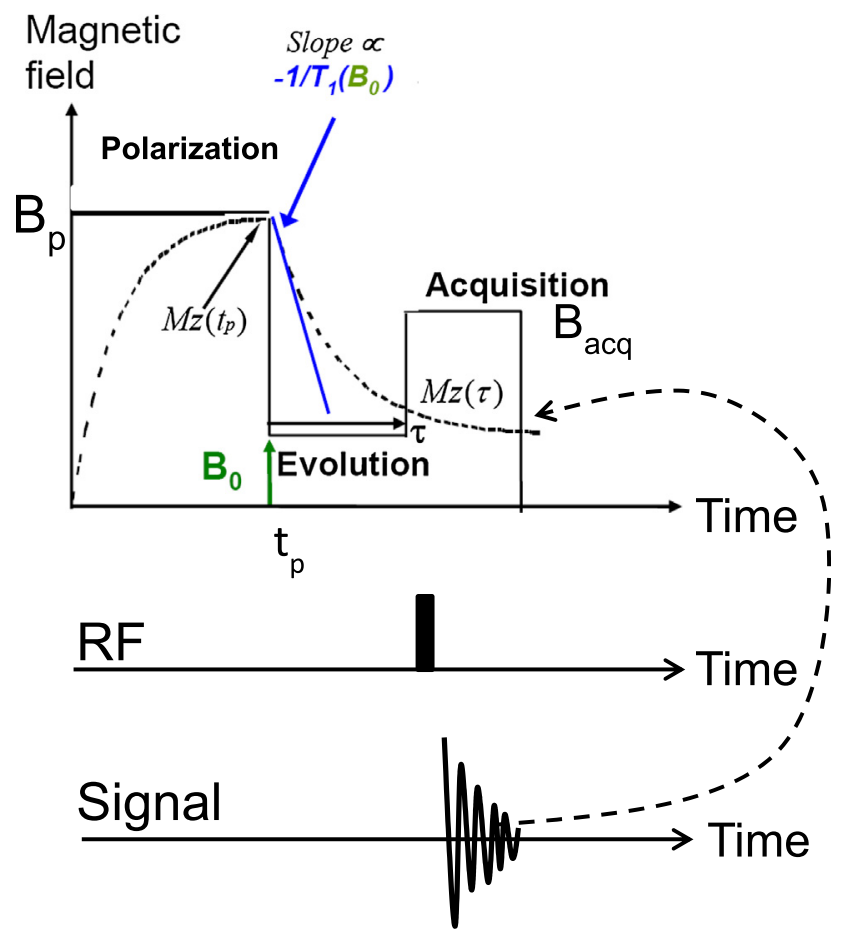

Fig. 2. Magnetic field cycle used in fast-field-cycling NMR sequence with Stelar instruments. Spins are pre-polarized at a magnetic field $B_{p}$ for a duration $t_{p}$, which is set to about $4 T_{1}$. The magnetic field is switched very fast ( $1-3 \mathrm{~ms}$ ) to a value $B_{0}$ and the nuclear magnetization allowed decaying during an evolution period of duration $\tau$. The field is then changed to a value $B_{a c q}$ and, the free-induction decay recorded following a single $90^{\circ}$ excitation pulse of $\sim 5.8 \mu$ s duration (for ${ }^{1} \mathrm{H}$ ). Generally, the experiment is repeated over a large range of proton Larmor frequencies $(10 \mathrm{kHz}-40$ $\mathrm{MHz}$ ) in order to obtain a complete dispersion profile for $1 / T_{1}$.

the so-called pre-polarized (PP) sequence (used for frequencies between $10 \mathrm{kHz}$ and $12 \mathrm{MHz}$ ) which allows improving the signal to noise ratio by pre-polarizing first the spins at a relatively high magnetic field $B_{p}$ (generally $30-40 \mathrm{MHz}$ for protons). The polarisation period allows acquiring a Curie equilibrium longitudinal magnetization after a certain number of spin-lattice relaxation times (generally $t_{p}=4 T_{1}$ ). As the polarisation field is created by current in a coil, then it is necessary to switch the current from a few hundred Amperes to almost $0 \mathrm{~A}$ to reach a relaxing field where the relaxation is studied. This requires an accurate cooling device for dissipating almost several $\mathrm{kW}$ of power in a $1 \mathrm{~ms}$ or so. The fastfield cycling (FFC) procedure permits to switch electronically in 1-3 ms this change with accuracy and stability. The sample then relaxes in the relaxing field which can be set to any desired value (down to a magnetic field of the order of 5 times the earth magnetic field e.g. $10 \mathrm{kHz}$ for ${ }^{1} \mathrm{H}$ ) on which the spin-lattice relaxation is desired. Finally, the field is set back to the detection field $B_{a c a}$ (acquisition field) in a very fast switching time (1-3 ms) for recording the free-induction decays following a single $90^{\circ}$ excitation pulse (of $\sim 5.8 \mu$ s duration for ${ }^{1} \mathrm{H}$ ); this field $B_{\text {acq }}$ corresponds to the r.f. tuning circuit. There is another complementary nonpolarized (NP) sequence in the high frequency range (12-40 $\mathrm{MHz}$ ) for protons. In a (NP) sequence (for frequencies between 12 and $40 \mathrm{MHz}$ ) $B_{a c q}$ is set more or less in the middle of the field range to assure similar switching time to ramp up or down to reach the acquisition field. In a (PP) sequence (Fig. 2), $B_{a c q}$ is slightly lower than $B_{p}$, but this not a general rule. In principle, it is possible to use a higher value for $B_{a c q}$ in order to boost the signal to noise $(\mathrm{S} / \mathrm{N})$, both in $(\mathrm{PP})$ and $(\mathrm{NP})$ sequences, while limiting the necessary switching time. The total experimental FFC procedure is repeated automatically for different relaxation evolution times $\tau$ (Fig. 2) on a large range of proton Larmor frequencies $(10 \mathrm{kHz}-40 \mathrm{MHz})$ in successive measurements, so as to yield complete NMRD profiles of $1 / T_{1}$. Stelar also make recently a FFC instrument based on a cryogen-free superconducting magnet that allows extension of the magnetic field strength range for the acquisition of NMRD profiles of protons at up to $120 \mathrm{MHz}$. Further details about this technique can be found in Refs. [5,6]. 
Since the pioneering works of Redfield [7] and Bryant [8,9], there is a complementary way to FFC NMRD for measuring the NMRD profiles at much higher magnetic fields. This is the pneumatic shuttle NMR where the sample moves physically between regions of different magnetic fields instead of leaving the sample stationary and switching the field as in FFC. This alternative technique allows studying the dynamics of native proteins in presence of long $T_{1}$. Redfield has proposed a shuttling field cycling device as a removable module inside the cryo-magnet of a commercial 500 $\mathrm{MHz}$ NMR instrument [10]. Bryant has proposed to shuttle the sample between two magnetically isolated independent magnets where the high field is made by a superconducting magnet providing a high resolution magnetic field of $7.05 \mathrm{~T}$, while the second electro-magnet, positioned below at $82 \mathrm{~cm}$ is in an iron shield [9]. Recently, some new developments were proposed for boosting sensitivity in high resolution sample shuttling relaxometry by using a cryo-probe and a fast mechanical sample shuttle device installed in commercial spectrometers of $500-700 \mathrm{MHz}$ working at several magnetic fields $[11,12]$. Combining relaxation data at various fields with high-resolution NMR data provides information from different nuclei on fast protein dynamics (long $T_{1}$ ) associated with structural information. The shuttle NMR is thus useful for studying fast molecular motions on proteins while the FFC NMRD focuses on slow molecular motions on liquids embedded in microporous media. Both NMR techniques are thus complementary.

Systems typically studied by NMRD, and which will be covered in this review, mainly comprise high surface-area microporous materials [13-15] chromatographic supports [15,16], heterogeneous catalytic materials [17], plasters [18,19], concretes [20], cement pastes [21], natural microporous organic kerogens and clay minerals in shale oils [22]. Standard NMR techniques at constant magnetic field have been also proposed for probing the molecular dynamics at pore surfaces despite the very low fraction of molecules in a surface layer. For instance, the transverse and longitudinal relaxation rate measurements for saturated calibrated microporous silica glasses have allowed separation of surface and bulk contributions $[13,14]$ thus giving information on the surface reorientational dynamics [23]. A progressive saturation of cement has permitted isolation of the surface contribution to spin-spin relaxation rates and allowed the validity of the biphasic fast exchange model in porous media to be checked by the linear dependence of transverse relaxation times with the fluid saturation factor [24]. However, the FFC NMR relaxation technique is more sensitive for probing directly the surface dynamics of embedded liquids in fully saturated pores, providing that a theory exists for studying the micro-dynamical behaviour of the liquid. For instance, the FFC technique has shown striking differences between aprotic [15] and protic [16] (water) liquids in contact with high surface-area microporous glass surfaces containing trace paramagnetic impurities. Numerous applications of FFC relaxation technique are reviewed to macroporous systems with low surface to volume ratio, such as granular packings [25,26] and oil-bearing rocks [27]. A particular emphasis of this review concerns the extension of the FFC NMR technique to industrial applications, for instance, for directly probing the dynamics of proton species at pore surfaces and specific surface areas of cement-based materials [20]. Similar methods gave information about the microstructure time-evolution of hydrated cement pastes [28]. Low field two-dimensional correlation NMR relaxation studies: $T_{1}-T_{2}[29]$ and $T_{2}-Z$-store- $T_{2}[30]$ have evidenced exchange of water between the connected micropores of white cement paste. The $T_{2}-z$-store- $T_{2}$ spin correlation sequence has been also applied to evidence water exchange in porous sandstone rocks [31], smectite gels with clay [32] and water and oil in rocks [33]. NMRD relaxometry techniques have also been proposed as a valid alternative to the traditional contact angle (CA) measurements for biochar water-wettability evaluation [34].
Another domain of interest has concerned biological applications. For instance, the FFC relaxation technique has proven useful for probing the interstitial translational diffusion of water using residual water-proton magnetic relaxation dispersion profile obtained from suspensions of phospholipid vesicles in deuterium oxide [35]. Numerous field-cycling relaxometry studies of protein solutions exist in the literature (see the review in Ref. [36]). Interesting studies for diamagnetic species were proposed in the review in Ref. [5]. For instance, proton spin-lattice relaxation induced by paramagnetic centers is dramatically enhanced for rotationally immobilized protein [37]. For these immobilized proteins rare binding sites for water in protein systems add very low frequency components to the dynamics spectrum. Water binding sites in protein systems are not identical, thus, distributions of free energies and consequent dynamics are expected. Deuterated water NMRD has characterized the local rotational fluctuations for proteinbound water molecules down to the $\mathrm{kHz}$ range. To account for the NMRD data at different temperatures, it has been shown that the extreme-values statistics of rare events, i.e., water dynamics in rare binding sites, implies an exponential distribution of activation energies for the strongest binding events [38]. Probing the water diffusional dynamics at the protein interface is critically important for characterizing the energies of interaction between proteins and substrates. Here, the magnetic field dependence of the water-proton $1 / T_{1}$ is characteristic of a 2 -dimensional diffusion of water in the protein interfacial region [39] with an average surface-translational correlation time of $30-40$ ps. The NMRD of the composite proton-water $T_{1}$ was measured for excised samples of rat liver, muscle, and kidney over the field range from 0.7 to $7 \mathrm{~T}(35-300 \mathrm{MHz})$ with an NMR sample-shuttle methods [40] exhibiting a translational correlation times of approximately 15 ps. In vivo MRI measurements of $T_{1}$ have shown that the translational correlation times of white matter are three times larger than those of grey matter tissues [41]. This analysis of brain tissues is consistent with diffusion-weighted MRI measurements reported by Le Bihan et al. [42].

This review outlines some recent examples showing how nuclear magnetic relaxation dispersion techniques allow characterizing fundamental properties that depend on the nature of the liquid and on its affinity for the pore surface such as surface correlation times, diffusion coefficients and dynamical surface affinity (NMR wettability). The focus is on the multi-frequency and multi-dimensional nuclear magnetic relaxation (NMR) techniques (Fig. 1) for probing these dynamical properties in calibrated and natural porous materials. Following this introduction, Section 2 outlines the main relaxation equations for describing the rotational and translational relaxation processes of a bulk liquid [43-46]. Section 3 describes the biphasic fast exchange conditions for relaxation of a liquid in proximity to a solid surface. Section 4 presents the two limiting nuclear magnetic relaxation processes of a liquid in pores [25]. Section 5 describes the striking differences between the relaxation NMRD profile of the same liquid in bulk and confinement [47]. Section 6 presents the basic relaxation equations of nuclear magnetic relaxation dispersion of $1 / T_{1}$ in calibrated micropores in turn for aprotic [15] and protic [16] liquids embedded in calibrated pores, while Section 7 outlines the first application of the multiscale NMRD relaxation investigation of microstructure evolution (ageing) of various cement-based materials $[20,21]$. This section also presents an application of NMRD for probing directly the nano-wettability of plaster pastes under various conditions of preparation $[18,19]$. Section 8 extends the analysis of NMRD profiles for separating oil and water contributions in real multimodal macroporous petroleum rocks [27], focussing on the separation of the dynamics and wettability of oil and water in the organic and mineral dual microporosity of shale oil rocks [22]. Section 9 covers some applications of FFC NMR for probing 
the molecular dynamics in macromolecules and proteins. An application of FFC relaxometry is shown on water dynamics at the surface of various macromolecules and proteins [37,38]. Last, the application to NMRD in biological tissues of animals and in vivo MRI of human brains is briefly described [40].

\section{Basics of nuclear magnetic relaxation dispersion in bulk}

The nuclear magnetic relaxation of protons in bulk liquids is a stimulated process induced by the coupling between the nuclear spins $(I=1 / 2)$ and the magnetic noise created by the molecular dynamics of the spin-bearing molecules $[43,44]$. If one assumes a stochastic independence of rotational and translational molecular diffusion, the frequency dependence of the overall spin-lattice relaxation $1 / T_{1}\left(\omega_{I}\right)$ is given by the superposition of intramolecular and intermolecular dipole-dipole contributions between "like" or "unlike" spins in diamagnetic samples:

$\frac{1}{T_{1, \text { bulk }}\left(\omega_{I}\right)}=\frac{1}{T_{1, \text { bulk }}^{\text {intra }}\left(\omega_{I}\right)}+\frac{1}{T_{1, \text { bulk }}^{\text {inter }}\left(\omega_{I}\right)}$.

These two contributions are induced by dipolar fluctuations occurring on different time-scales. The former comes from fast molecular reorientations while the latter comes from molecular translation over spatial distances much longer than the molecular sizes. Usually, these two contributions can be separated by using deuterated molecules of the same species where the frequency dependence of the deuteron $1 / T_{1}$ is largely dominated by the intramolecular contribution.

\subsection{Intramolecular relaxation by isotropic rotational diffusion}

Bloembergen, Purcell and Pound (BPP) introduced the following longitudinal and transverse relaxation equations in the case of an isotropic rotational diffusion between two-spins $I=1 / 2$ [44]:

$\frac{1}{T_{1, \text { bulk }}^{\text {intra }}\left(\omega_{I}\right)}=\left(\frac{\mu_{0}}{4 \pi}\right)^{2} \frac{\gamma^{4} \hbar^{2}}{8}\left[J\left(\omega_{I}\right)+4 J\left(2 \omega_{I}\right)\right]$,

$\frac{1}{T_{2, \text { bulk }}^{\text {intra }}\left(\omega_{I}\right)}=\left(\frac{\mu_{0}}{4 \pi}\right)^{2} \frac{\gamma^{4} \hbar^{2}}{16}\left[3 J(0)+5 J\left(\omega_{I}\right)+2 J\left(2 \omega_{I}\right)\right]$.

Here $\gamma$ is the gyromagnetic ratio and $\hbar$ is the Planck's constant divided by $2 \pi, \omega_{I}=\gamma B_{0}$. The spectral density $J\left(\omega_{I}\right)$ is defined as the Fourier transform of an isotropic exponential dipolar correlation function $G(\tau)$ obtained from an average over an orientational conditional probability that is solution of the rotational diffusion equation:

$G(\tau)=\frac{6}{5 b^{6}} \exp \left(-\frac{|\tau|}{\tau_{c}}\right)$,

$J\left(\omega_{I}\right)=\frac{6}{5 b^{6}} \frac{2 \tau_{c}}{1+\omega_{I}^{2} \tau_{c}^{2}}$,

where $b$ is the nuclear inter-spin distance within the same molecule. Substituting Eqs. (3a, b) into Eqs. (2a, b) gives the following basic relaxation equations for a bulk liquid:

$\frac{1}{T_{1, \text { bulk }}^{\text {intra }}\left(\omega_{I}\right)}=\left(\frac{\mu_{0}}{4 \pi}\right)^{2} \frac{3 \gamma^{4} \hbar^{2}}{10 b^{6}} \tau_{c}\left[\frac{1}{1+\omega_{I}^{2} \tau_{c}^{2}}+\frac{4}{1+4 \omega_{I}^{2} \tau_{c}^{2}}\right]$,

$\frac{1}{T_{2, \text { bulk }}^{\text {intra }}\left(\omega_{I}\right)}=\left(\frac{\mu_{0}}{4 \pi}\right)^{2} \frac{3 \gamma^{4} \hbar^{2}}{20 b^{6}} \tau_{c}\left[3+\frac{5}{1+\omega_{I}^{2} \tau_{c}^{2}}+\frac{2}{1+4 \omega_{I}^{2} \tau_{c}^{2}}\right]$.

For a spherical molecule of radius $R$ the correlation time $\tau_{c}$ is given by the Stokes-Einstein relation: $\tau_{c}=\frac{4 \pi \eta R^{3}}{3 k_{B} T}$

where $\eta$ is the viscosity, $k_{B}$ is the Boltzmann factor and $T$ is the temperature. Considering the particular case of water $R \sim b \sim 0.15 \mathrm{~nm}$, $\eta=1 \mathrm{cP}$ and $\tau_{c}=3.5 \mathrm{ps}$ at room temperature, one finds a frequencyindependent value $T_{1, \text { bulk }}^{\text {intra }}\left(\omega_{I}\right)=T_{2, \text { bulk }}^{\text {intra }}\left(\omega_{I}\right) \approx\left(\frac{4 \pi}{\mu_{0}}\right)^{2} \frac{k_{B} T R^{3}}{2 \pi \gamma^{4} \hbar^{2} \eta} \approx 3.8 \mathrm{~s}$ in the range of extreme narrowing $\left(\omega_{I} \tau_{c} \ll 1\right)$.

\subsection{Intermolecular relaxation by translational diffusion}

Analytical expressions of the intermolecular contributions of the relaxation rates coming from the translational diffusion have been considered originally by Hubbard [45]. However, Ayant et al. [46] have corrected these analytical expressions in the high frequency range by considering the right boundary condition of no diffusive flux at the surface of a hard sphere. Hwang and Freed found similar expressions with a different mathematical treatment [48]. These analytical expressions are formally given by:

$$
\begin{aligned}
& \frac{1}{T_{1, b u l k}^{\text {inter }}\left(\omega_{I}\right)}=\left(\frac{\mu_{0}}{4 \pi}\right)^{2} \frac{6 \pi}{5} N \frac{\gamma^{4} \hbar^{2}}{d^{3}} \tau_{t}\left[I\left(\omega_{I}\right)+4 I\left(2 \omega_{I}\right)\right], \\
& \frac{1}{T_{2, \text { bulk }}^{\text {inter }}\left(\omega_{I}\right)}=\left(\frac{\mu_{0}}{4 \pi}\right)^{2} \frac{3 \pi}{5} N \frac{\gamma^{4} \hbar^{2}}{d^{3}} \tau_{t}\left[3 I(0)+5 I\left(\omega_{I}\right)+2 I\left(2 \omega_{I}\right)\right],
\end{aligned}
$$

where $N$ is the number of spins per unit of volume and:

$$
\tau_{t}=\frac{12 \pi \eta R^{3}}{k_{B} T}=9 \tau_{c}
$$

$$
\begin{aligned}
I(u) & =\frac{(3 / 2) u^{2}+(15 / 2) u+12}{(1 / 8) u^{6}+u^{5}+4 u^{4}+(27 / 2) u^{3}+(81 / 2) u^{2}+81 u+81} \text { with } u \\
& =\sqrt{2 \omega_{I} \tau_{t}}
\end{aligned}
$$

Substituting Eq. (8) into Eqs. (6a, 6b), gives the following asymptotic relaxation rates in the low and high frequency ranges:

$$
\begin{aligned}
\frac{1}{T_{1, \text { bulk }}^{\text {inter }}\left(\omega_{I}\right)} \approx\left(\frac{\mu_{0}}{4 \pi}\right)^{2} \frac{8 \pi}{9} N \frac{\gamma^{4} \hbar^{2}}{d^{3}} \tau_{t}\left[1-\frac{3(\sqrt{2}+8)}{40} \sqrt{\omega_{I} \tau_{t}}+\cdots\right] \\
\text { when } \quad \omega_{I} \tau_{t} \ll 1 \\
\frac{1}{T_{1, \text { bulk }}^{\text {inter }}\left(\omega_{I}\right)} \approx\left(\frac{\mu_{0}}{4 \pi}\right)^{2} \frac{18 \pi}{5} N \frac{\gamma^{4} \hbar^{2}}{d^{3}} \omega_{I}^{-2} \tau_{t}^{-1}+\cdots \quad \text { when } \omega_{I} \tau_{t} \gg 1 \\
\frac{1}{T_{2, \text { bulk }}^{\text {inter }}\left(\omega_{I}\right)} \approx\left(\frac{\mu_{0}}{4 \pi}\right)^{2} \frac{8 \pi}{9} N \frac{\gamma^{4} \hbar^{2}}{d^{3}} \tau_{t}\left[1-\frac{3}{160}(3+10 \sqrt{2}) \sqrt{\omega_{I} \tau_{t}}\right] .
\end{aligned}
$$

Here $\tau_{t}=d /(2 D)$ where $d \sim 2 R$ is the distance of minimal approach between two spherical molecules of radius $R$ and $D$ is the translational diffusion coefficient given by the Stokes-Einstein relation $D=\frac{k_{B} T}{6 \pi \eta R^{2}}$. Substituting the intramolecular (Eqs. 4) and intermolecular (Eqs. (6)-(8)) contributions of longitudinal relaxation rates into Eq. (1) gives for water a NMRD profile of the overall longitudinal relaxation rate that is almost constant $\frac{1}{T_{1, \text { bulk }}\left(\omega_{I}\right)}=\frac{1}{T_{1, \text { bulk }}^{\text {intra }}\left(\omega_{I}\right)}+\frac{1}{T_{1, \text { bulk }}^{\text {inter }}\left(\omega_{I}\right)} \approx 0.29 \mathrm{~s}^{-1}$ in the whole frequency range $\left(10^{-2}-40 \mathrm{MHz}\right)$ studied with the FFC spectrometer. The observed NMRD profiles of bulk water and oil (dodecane) at room temperature are displayed in Fig. 3. The frequency independence for water and oil observed in Fig. 3 reflects the fast molecular dynamics of these bulk liquids. For water, one finds a $1 / T_{1, \text { bulk }}=0.45 \mathrm{~s}^{-1}$ which slightly differs from the theoretical value given above. This is due 


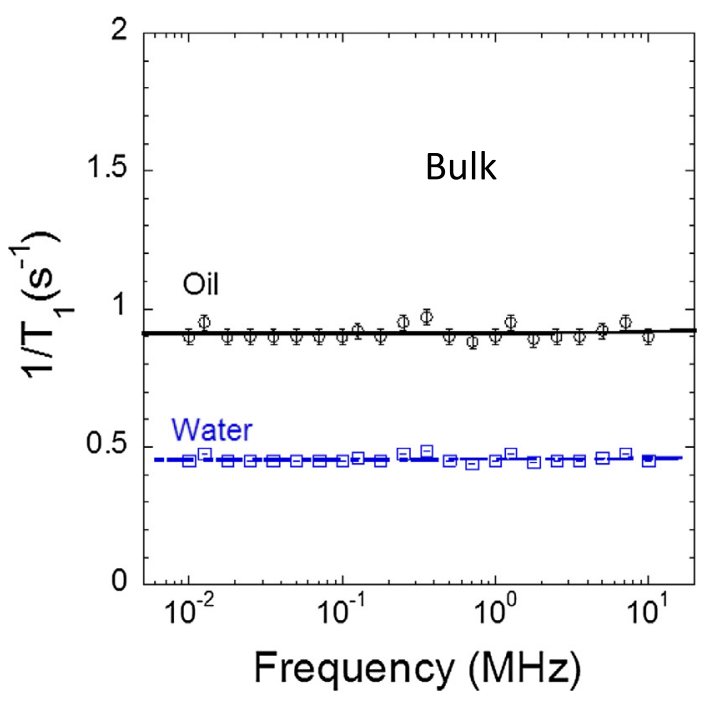

Fig. 3. Measured bulk water and oil (dodecane) spin-lattice relaxation rates as a function of the proton Larmor frequency (data from [47]).

to the paramagnetic contribution of the oxygen naturally dissolved in water that shortens the $T_{1}$ value; bubbling nitrogen through the sample causes the observed $T_{1}$ value to increase to the theoretical value. The factor 2 observed between the relaxation rates $1 / T_{1}$ of oil and water (Fig. 3 ) is due to their difference in correlation times, molecular sizes and densities.

\subsection{Quadrupolar nuclear relaxation of isotropically rotating molecules}

The quadrupolar relaxation process that occurs for nuclear spins with $I>1 / 2$ is due to the coupling between the nuclear quadrupole moment $Q$ and the fluctuating local molecular electric field gradient eq. There are many reasons to consider such a relaxation process. For instance, several NMR studies on the dynamics of liquid crystals are concerned with the deuterium nuclei of $\operatorname{spin} I=1$ [49-54]. This is a very useful nucleus for studying the different dynamical motions in the lipid bilayers and biological membranes [54]. It has been also used for probing the anisotropic dynamics of liquids confined in nanopores [23]. There are also numerous in vivo NMR studies of metallic ions for separating the intra- and extracellular ${ }^{23} \mathrm{Na}[55]$.

An interesting situation is encountered when using deuterated molecules for separating the intra- and intermolecular contributions in the relaxation of bulk liquids [56]. In the extreme narrowing regime $\left(\omega_{0} \tau_{c} \ll 1\right)$, the ${ }^{2} \mathrm{H}$ spin-lattice relaxation rate for molecules tumbling isotropically is given by:

$\frac{1}{T_{1, \text { bulk }}^{\text {intra }}} \approx\left(\frac{\mu_{0}}{4 \pi}\right)^{2} \frac{3}{8}\left(1+\frac{\eta_{A}^{2}}{3}\right)\left(\frac{e^{2} q Q}{\hbar}\right)^{2} \tau_{c}$,

where $\eta_{A}$ is the asymmetry parameter of the field gradient $e q\left(\eta_{A}=0\right.$ for an axial symmetry of the electric field gradient), $Q$ is the nuclear electric quadrupolar moment and the rotational correlation time $\tau_{\mathrm{c}}$ is given in Eq. (5). We note from Eq. (10) that the relaxation of deuterons is thus dominated by intramolecular interactions with local electric field gradients. Due to the large values of the ${ }^{2} \mathrm{H}$ quadrupolar coupling constant (about $170 \mathrm{kHz}$ ) in Eq. (10), the quadrupolar and in consequence the intramolecular contribution dominates when using perdeuterated molecules of the same kind. It thus becomes possible to separate by NMR the intermolecular and intramolecular contributions.

\section{Biphasic fast-exchange relations}

The influence of molecular motions on the NMR line shape has been studied for a long time. A special case of molecular motion concerns conformational changes in molecules and chemical exchange between different sites. McConnell extended the classical NMR Bloch equations to include consideration of chemical exchange [57], and Goldman has extended the analysis further to analyse the shape of the NMR FID in the case where the correlation times of the molecular motions $\tau_{c} \sim 1 / \Delta \omega_{0}$ are of the order of the inverse NMR linewidth $\Delta \omega_{0}[58,59]$. For that purpose, he introduced a method based on the sudden jump approximation for random exchange jumps based on the Poisson probability distribution function (pdf) for describing different types of exchange-driven modulations through molecular conformational changes and intermolecular exchange [58,59]. An application of this method has been proposed for characterizing the closed porosity of porous hybrid organic-inorganic sol-gel matrices by means of measuring ${ }^{2} \mathrm{H}$ nuclear relaxation of d16-adamantane confined in the network of progressively closing pores that exists during drying of such a gel [60]. Due its importance in this review, the main steps of this method are described in detail for proving the well-known expressions of the longitudinal nuclear spin relaxation rates of a liquid confined in pores in presence of surface-bulk exchange.

\subsection{Evolution of the density matrix in presence of exchange between two spin states}

In Liouville space, there are super operators $\widehat{\widehat{H}}$ (commutators in Schrödinger space) and the density operator $\hat{\sigma}$ which is a vector follows the following equation [61]:

$$
\frac{d \hat{\sigma}}{d t}=-i \widehat{\hat{H}} \hat{\sigma}-\widehat{\Gamma}\left(\widehat{\sigma}-\widehat{\sigma}_{e q}\right)
$$

Here $\widehat{H}$ is a coherent superoperator and $\widehat{\Gamma}$ is a relaxation superoperator. Jeener has transformed Eq. (11) to a linear matrix form that contains both the coherent and incoherent (relaxation) in the same superoperator $\widehat{\bar{\Pi}}[61,62]$ :

$$
\frac{d \hat{\sigma}}{d t}=-\widehat{\widehat{\Pi}} \hat{\sigma} \text {. }
$$

A similar method has been used more recently (see Eq. (84) of Ref. [62]). In Eq. (12) $\widehat{\sigma}$ can be transformed in $\left(\widehat{\sigma}-\widehat{\sigma}_{e q}\right)$, where $\widehat{\sigma}_{e q}$ is the density operator at equilibrium in order to describe the evolution to equilibrium at a finite temperature [43]. The formal solution of Eq. (12) is thus:

$\widehat{\sigma}(t)=\exp [-\widehat{\widehat{\Pi}} t] \widehat{\sigma}(0)$.

Consider a spin system that undergoes sudden exchanges between two spin states labeled 1 and 2 at a time $\tau_{i}(i \in(1,2))$ characteristic of this exchange. One assumes that the duration for which the system persists in a given state follows a Poisson distribution function $(p d f)$ :

$P_{n}(t)=\frac{(\lambda t)^{n}}{n !} e^{-\lambda t}$

where $\lambda=1 / \tau_{i}$ is the inverse of the exchange time and $n$ is the number of steps. In other words, if the system is in state 1 at $t=0$, the probability of having no motion $(n=0)$ between 0 and $t$ is

$P_{0}(t)=e^{-\lambda_{1} t}$. 
We are interested in following the evolution in time $t$ of the density operator initially prepared in state 1 by a $\pi / 2$ pulse. The alternative of initial preparation in state 2 will be considered later. The proposed treatment follows that of Goldman [58,59], but considers the density operator instead of the FID. According to Eqs. (13)-(15), the fraction of density operator coming from those spins that do not change between states $1 \rightarrow 2$ during the time interval 0 to $t$ is:

$e^{-\lambda_{1} t} \exp \left[-\widehat{\bar{\Pi}}_{1} t\right] \widehat{\sigma}(0)$.

Now, the fraction of density operator coming from spins that change state only one time between $1 \rightarrow 2$ during the time interval 0 to $\mathrm{t}$ is after integration:

$\int_{0}^{t} \lambda_{1} d t^{\prime} \exp \left[-\lambda_{2}\left(t-t^{\prime}\right)\right] \exp \left[-\widehat{\bar{\Pi}}_{2}\left(t-t^{\prime}\right)\right] \exp \left(-\lambda_{1} t^{\prime}\right) \exp \left[-\widehat{\bar{\Pi}}_{1} t\right] \widehat{\sigma}(0)$

The order of terms in Eq. (17) is important due to the irreversibility and the possible non-commutativity of operators. The generalization of Eq. (17) to the case of 2 changes of states $1 \rightarrow 2 \rightarrow 1$ between times 0 and $t$ is:

$\int_{0}^{t} \lambda_{2} d t^{\prime} \exp \left[-\lambda_{1}\left(t-t^{\prime}\right)\right] \exp \left[-\widehat{\Pi}_{1}\left(t-t^{\prime}\right)\right]$

$\times \int_{0}^{t^{\prime}} \lambda_{1} d t^{\prime \prime} \exp \left[-\lambda_{2}\left(t^{\prime}-t^{\prime \prime}\right)\right] \exp \left[-\widehat{\bar{\Pi}}_{2}\left(t^{\prime}-t^{\prime \prime}\right)\right] \exp \left(-\lambda_{1} t^{\prime \prime}\right) \exp \left[-\widehat{\Pi}_{1} t^{\prime \prime}\right] \widehat{\sigma}(0)$

The process can be continued iteratively. Here, the use of the Laplace transform simplifies the problem greatly. As usual in this iterative process, it is well known that the Laplace transform of the density operator may be calculated by introducing the notation $\widehat{G}(z)$ defined as:

$\tilde{\widehat{\sigma}}(z)=\int_{0}^{\infty} d t \exp [-z t] \widehat{\sigma}(t) \equiv \widehat{\widehat{G}}(z) \widehat{\sigma}(0)$,

Substituting Eq. (13) into Eq. (19) gives immediately:

$\tilde{\widehat{\sigma}}(z)=\int_{0}^{\infty} d t \exp [-z t] \exp [-\widehat{\widehat{\Pi}} t] \widehat{\sigma}(0) \equiv \widehat{\bar{G}}(z) \hat{\sigma}(0)$.

With this definition and owing to the property of a Laplace transform of a convolution product, Eq. (17) becomes

$\lambda_{1} \widehat{\widehat{G}}_{2}\left(z+\lambda_{2}\right) \widehat{\bar{G}}_{1}\left(z+\lambda_{1}\right) \hat{\sigma}(0)$

Similarly Eq. (18) becomes:

$\lambda_{2} \widehat{\widehat{G}}_{1}\left(z+\lambda_{1}\right) \lambda_{1} \widehat{\widehat{G}}_{2}\left(z+\lambda_{2}\right) \widehat{\widehat{G}}_{1}\left(z+\lambda_{1}\right) \sigma(0)$

Repeating the same operations gives after an even $(2 p)$ number of times:

$\widehat{\widehat{G}}_{1}\left(z+\lambda_{1}\right)\left[\lambda_{1} \lambda_{2} \widehat{\widehat{G}}_{2}\left(z+\lambda_{2}\right) \widehat{\bar{G}}_{1}\left(z+\lambda_{1}\right)\right]^{p} \widehat{\sigma}(0)$

and after an odd number $(2 p+1)$ :

$\lambda_{1} \widehat{\widehat{G}}_{2}\left(z+\lambda_{2}\right) \widehat{\widehat{G}}_{1}\left(z+\lambda_{1}\right)\left[\lambda_{1} \lambda_{2} \widehat{\widehat{G}}_{2}\left(z+\lambda_{2}\right) \widehat{\widehat{G}}_{1}\left(z+\lambda_{1}\right)\right]^{p} \widehat{\sigma}(0)$

Summing over all the different steps and with introduction of the identity superoperator $\widehat{I}_{d}$, the Laplace transform of the density operator initially in state 1 is:

$$
\begin{aligned}
& {\left[\widehat{\widehat{I}}_{d}+\lambda_{1} \widehat{\widehat{G}}_{2}\left(z+\lambda_{2}\right)\right] \widehat{\widehat{G}}_{1}\left(z+\lambda_{1}\right)} \\
& \quad \times\left\{\sum_{p=0}^{\infty}\left[\lambda_{1} \lambda_{2} \widehat{\widehat{G}}_{2}\left(z+\lambda_{2}\right) \widehat{\widehat{G}}_{1}\left(z+\lambda_{1}\right)\right]^{p}\right\} \hat{\sigma}(0)
\end{aligned}
$$

By permutation of indices 1 and 2, and following the same treatment, one obtains the Laplace transform of the density operator initially in state 2 :

$\left[\widehat{\widehat{I}}_{d}+\lambda_{2} \widehat{\widehat{G}}_{1}\left(z+\lambda_{1}\right)\right] \widehat{\widehat{G}}_{2}\left(z+\lambda_{2}\right)\left\{\sum_{p=0}^{\infty}\left[\lambda_{1} \lambda_{2} \widehat{\widehat{G}}_{1}\left(z+\lambda_{1}\right) \widehat{\widehat{G}}_{2}\left(z+\lambda_{2}\right)\right]\right\} \hat{\sigma}(0)$

It is easy to establish the following formal form by considering a basis of eigenvectors of $\widehat{\Pi}$

$\left.\int_{0}^{\infty} d t \exp [-z t] \exp [-\widehat{\hat{\Pi}} t] \equiv z \widehat{\widehat{I}}_{d}+\widehat{\widehat{\Pi}}\right)^{-1}$

Summing Eqs. (24a) and (24b) with use of Eq. (25), gives:

$$
\begin{aligned}
& {\left[\widehat{\widehat{I}}_{d}+\lambda_{1}\left(\left(z+\lambda_{2}\right) \widehat{\widehat{I}}_{d}+\widehat{\widehat{\Pi}}_{2}\right)^{-1}\right]\left(\left(z+\lambda_{1}\right){\widehat{I}}_{d}+\widehat{\widehat{\Pi}}_{1}\right)^{-1}} \\
& \times\left\{\sum_{p=0}^{\infty}\left[\lambda_{1} \lambda_{2}\left(\left(z+\lambda_{2}\right) \widehat{\widehat{I}}_{d}+\widehat{\widehat{\Pi}}_{2}\right) \quad\left(\left(z+\lambda_{1}\right) \widehat{\widehat{I}}_{d}+\widehat{\widehat{\Pi}}_{1}\right)^{-1}\right]\right\} \hat{\sigma}(0)+ \\
& \left.\widehat{\widehat{I}}_{d}+\lambda_{2}\left(\left(z+\lambda_{1}\right) \widehat{\bar{I}}_{d}+\widehat{\bar{\Pi}}_{1}\right)^{-1}\right]\left(\left(z+\lambda_{2}\right) \widehat{\bar{I}}_{d}+\widehat{\bar{\Pi}}_{2}\right) \\
& \times\left\{\sum_{p=0}^{\infty}\left[\lambda_{1} \lambda_{2}\left(\left(z+\lambda_{1}\right) \widehat{\widehat{I}}_{d}+\widehat{\widehat{\Pi}}_{1}\right)^{-1}\left(\left(z+\lambda_{2}\right) \widehat{\widehat{I}}_{d}+\widehat{\widehat{\Pi}}_{2}\right)^{-1}\right]\right\} \hat{\sigma}(0)
\end{aligned}
$$

After elementary calculations and omitting the super-operator notations for simplification, one obtains the following result:

$\tilde{\bar{\sigma}}(z)=\left\{\begin{array}{c}\left(z+\lambda_{2}+\Pi_{2}+\lambda_{1}\right)\left[\left(z+\lambda_{2}+\Pi_{2}\right)\left(z+\lambda_{1}+\Pi_{1}\right)-\lambda_{1} \lambda_{2}\right]^{-1}+ \\ \left(z+\lambda_{1}+\Pi_{1}+\lambda_{2}\right)\left[\left(z+\lambda_{1}+\Pi_{1}\right)\left(z+\lambda_{2}+\Pi_{2}\right)-\lambda_{1} \lambda_{2}\right]^{-1}\end{array}\right\} \hat{\sigma}(0)$.

Consider the simplified case where the two operators commute $\left[\Pi_{1}, \Pi_{2}\right]=0$; after multiplying the two terms by their respective probability $\tau_{\mathrm{i}} /\left(\tau_{1}+\tau_{2}\right)$, Eq. (27) becomes:

$$
\begin{aligned}
\tilde{\tilde{\sigma}}(z)= & \left\{\frac{\lambda_{2}}{\left(\lambda_{1}+\lambda_{2}\right)}\left(z+\lambda_{2}+\Pi_{2}+\lambda_{1}\right)+\frac{\lambda_{1}}{\left(\lambda_{1}+\lambda_{2}\right)}\left(z+\lambda_{1}+\Pi_{1}+\lambda_{2}\right)\right\} \\
& \times\left[\left(z+\lambda_{2}+\Pi_{2}\right)\left(z+\lambda_{1}+\Pi_{1}\right)-\lambda_{1} \lambda_{2}\right]^{-1} \tilde{\sigma}(0)
\end{aligned}
$$

Finally, introducing the equilibrium operator:

$\Pi_{e q}=\frac{\lambda_{2} \Pi_{1}+\lambda_{1} \Pi_{2}}{\lambda_{1}+\lambda_{2}}=\frac{\tau_{1} \Pi_{1}+\tau_{2} \Pi_{2}}{\tau_{1}+\tau_{2}}$,

Eq. (28) simplifies to the following non-exponential form:

$\tilde{\widehat{\sigma}}(z)=\left\{z+\Pi_{e q}+C(z)\right\}^{-1} \hat{\sigma}(0)$,

where the non-exponential contribution is:

$C(z)=\frac{-\lambda_{1} \lambda_{2}\left(\Pi_{1}-\Pi_{2}\right)^{2}}{\left(\lambda_{1}+\lambda_{2}\right)}\left[z\left(\lambda_{1}+\lambda_{2}\right)+\lambda_{1} \Pi_{1}+\lambda_{2} \Pi_{2}\right]^{-1}$. 
3.2. Biphasic fast exchange longitudinal relaxation equation

For the case of longitudinal relaxation, the operators $\Pi_{1}=1 / T_{1,1}$ and $\Pi_{2}=1 / T_{1,2}$ represent the longitudinal relaxation rates; in the case of a spin exchange between two spin states 1 and 2, Eq. (30) becomes:
$\tilde{\tilde{\sigma}}(z)=\left\{z+\frac{1}{T_{1, e q}}+C(z)\right\}^{-1} \widehat{\sigma}(0)$

where:

$\frac{1}{T_{1, e q}}=\frac{\tau_{1}}{\tau_{1}+\tau_{2}} \frac{1}{T_{1,1}}+\frac{\tau_{2}}{\tau_{1}+\tau_{2}} \frac{1}{T_{1,2}}$,

\section{Slow diffusion limit: strong surface relaxivity and/or large pores}

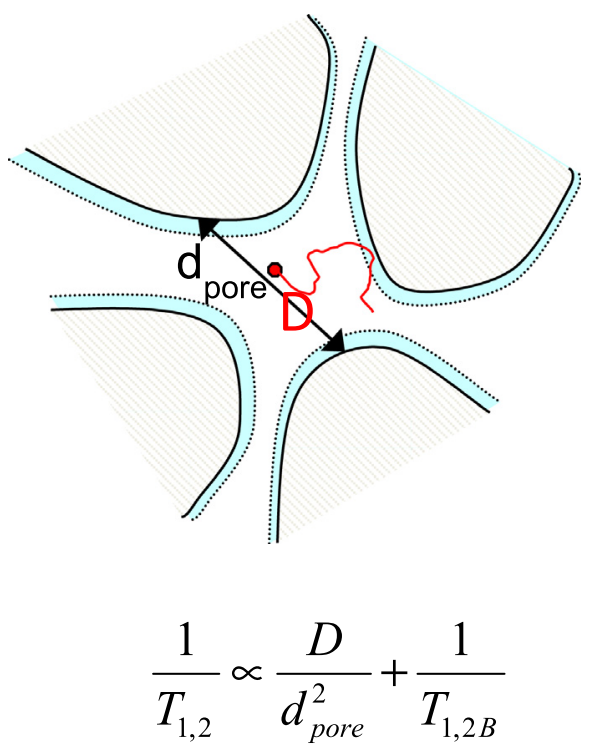

(a)

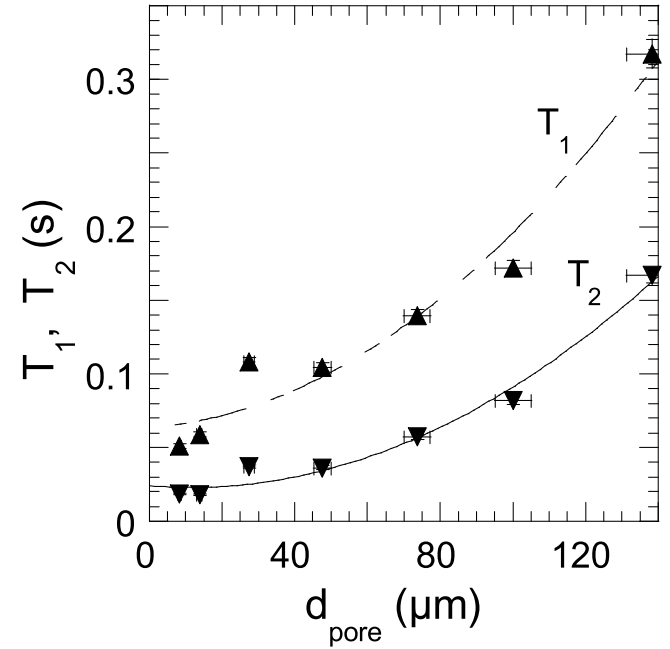

$T_{1}=>D=2.310^{-5} \mathrm{~cm}^{2} / \mathrm{s}$

Fast diffusion limit: weak surface relaxivity and / or small pores
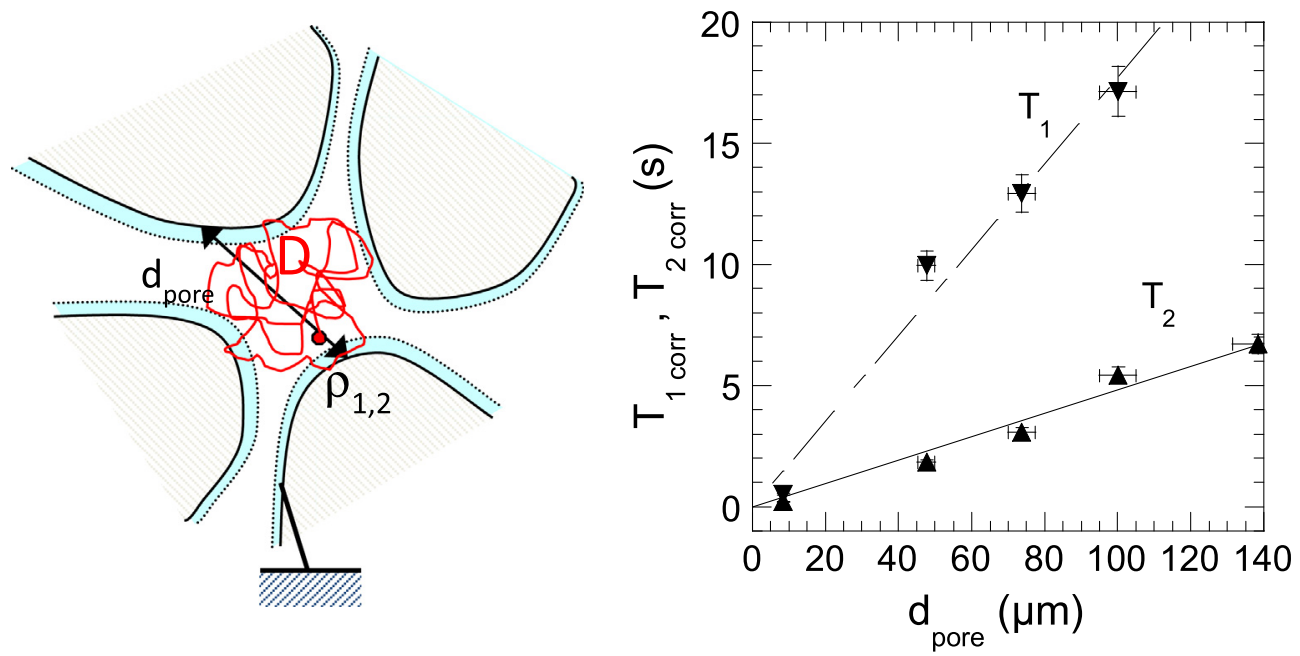

$$
\frac{1}{T_{1,2}}-\frac{1}{T_{1,2 B}} \propto \frac{\rho_{1,2}}{d_{\text {pore }}}
$$

$0.3 \leq \rho_{1} \leq 1 \mu \mathrm{m} / \mathrm{s}, 3 \leq \rho_{2} \leq 5 \mu \mathrm{m} / \mathrm{s}$.

(b)

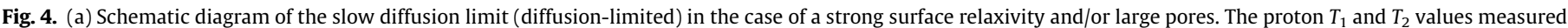

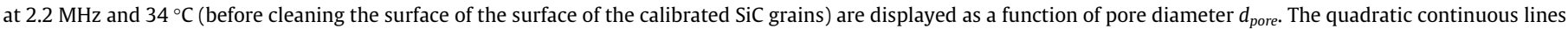

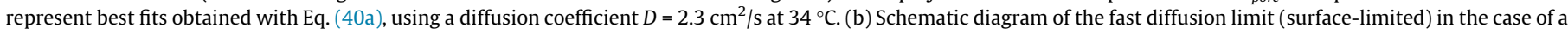

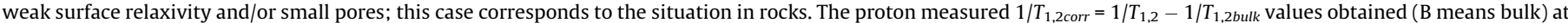

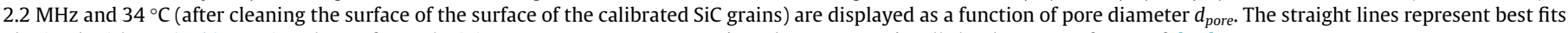
obtained with Eq. (40b), varying the surface relaxivity parameter $\rho_{1}=0.94 \mu \mathrm{m} / \mathrm{s}$ and $\rho_{2}=3.4 \mu \mathrm{m} / \mathrm{s}$. All the data come from Ref. [25]. 
$C(z)=\frac{-\lambda_{1} \lambda_{2}}{\left(\lambda_{1}+\lambda_{2}\right)}\left(\frac{1}{T_{1,1}}-\frac{1}{T_{1,2}}\right)^{2}\left[z\left(\lambda_{1}+\lambda_{2}\right)+\lambda_{1} \frac{1}{T_{1,1}}+\lambda_{2} \frac{1}{T_{1,2}}\right]^{-1}$.

$C(z)$ is a decreasing and positive function with a maximal value at $C(0)$. The exchange is considered fast and the relaxation becomes exponential when the correction factor $C(z)$ is negligible, when $C$ $(0) \ll 1 / T_{1, e q}$.

Let consider the biphasic fast exchange between two reservoirs of populations $N_{1} \gg N_{2}$ in the conditions where $\tau_{2} \ll \tau_{1}$ and $T_{1,1} \gg$ $T_{1,2}$. In other words, reservoir 1 has a larger population than reservoir 2 but it is less efficient for exchange. In that case, the longitudinal relaxation rate $1 / T_{1}=1 / T_{1, e q}+C(0)$ can be found easily from Eqs. (32)-(34) as

$\frac{1}{T_{1}}=1 / T_{1, e q}+C(0) \sim \frac{1}{T_{1,1}}+\frac{\tau_{2}}{\tau_{1}} \frac{1}{T_{1,2}+\tau_{2}}$

This is exactly the result found by McConnell [57]. This relation is widely used in the domain of paramagnetic relaxation agents for NMR imaging where the ratio is expressed in terms of metal complex concentration: $\tau_{2} / \tau_{1}=p_{m}$ [63].

In the following part of the review, we will be interested in the case of a biphasic fast exchange between the surface and the bulk relaxation contributions of a liquid in a pore. Since at steady state the fraction of spins that move from $1 \rightarrow 2$ is equal to those moving from $2 \rightarrow 1$ between times $t$ and $t+d t$, the following relation is verified:

$\frac{N_{1}}{N_{2}}=\frac{\tau_{1}}{\tau_{2}}$.

Introducing the new notations $N_{1} \equiv N_{\text {bulk }}$ and $N_{2} \equiv N_{\text {surf }}$ for labeling the spin populations of bulk and surface layer in pores and assuming that $N_{\text {bulk }} \gg N_{\text {surf }}$ as well as the conditions given above, Eq. (35) becomes:

$\frac{1}{T_{1}}=\frac{1}{T_{1, \text { bulk }}}+\frac{N_{\text {surf }}}{N_{\text {bulk }}+N_{\text {surf }}} \frac{1}{T_{1, \text { surf }}+\tau_{\text {surf }}}$,

which generalizes the relaxation equation of McConnell [57] to the relaxation of a liquid embedded in pores. For most applications, one can neglect the surface correlation time compared to the surface relaxation rate so that Eq. (37) finally becomes:

$\frac{1}{T_{1}}=\frac{1}{T_{1, \text { bulk }}}+\frac{N_{\text {surf }}}{N_{\text {bulk }}+N_{\text {surf }}} \frac{1}{T_{1, \text { surf }}}$

Eq. (38) corresponds to the well-known biphasic fast exchange relaxation of a liquid in pores considered by Brownstein and Tarr for different geometries of pores [64].

\section{Limiting nuclear magnetic relaxation processes of a liquid in pores}

What is the limiting nuclear magnetic relaxation process for a liquid in pores? Answering this question is important not only from an academic point of view but also for numerous industrial applications. Basically there are two limiting cases, namely those of relaxation under slow diffusion (diffusion-limited; bulk diffusion processes dominate) or fast diffusion (surface-limited; surface processes dominate) $[64,65]$. Analytical expressions for nuclear relaxation rates have been proposed for each of these limiting cases, and the general situation where both relaxation processes occur has been considered by Godefroy et al. [25]. In the condition of biphasic fast exchange, coupled Bloch equations including the surface and bulk relaxation contributions have been solved for liquids filling different pore geometries leading to the following relaxation equation [25]:
$\frac{1}{T_{1,2}}=\frac{1}{T_{1,2 b u l k}}+\left[\frac{d_{\text {pore }}}{2 \alpha \rho_{1,2}}+\frac{d_{\text {pore }}^{2}}{8 \alpha D}\right]^{-1}$.

In Eq. (39), $d_{\text {pore }}$ is the pore dimension (diameter) of the model pore and $\alpha=1,2$ or 3 is a shape factor for planar (pore-slit), cylindrical and spherical pore geometries, respectively. The efficiency of the surface spin-lattice $\left(\rho_{1}\right)$ or transverse $\left(\rho_{2}\right)$ relaxivities is qualified by $\rho_{1,2}=\lambda / T_{1,2 S}$ where $T_{1,2 S}$ represents the longitudinal or transverse surface relaxation times and the length scale $\lambda$ is a representative distance of the order of a few molecular sizes [66] (shown in light blue in Fig. 4a and b) beyond which the effects of the relaxation disappear. $D$ is the translational self-diffusion coefficient of the liquid within the pore. Eq. (39) separates clearly the bulk and confined relaxation contributions as two processes in parallel. However, within the contribution from confined relaxation in Eq. (39) there are two relaxation processes in series. A direct comparison of $4 D / d_{\text {pore }}$ and $\rho_{1,2}$ shows that the slowest process limits the relaxation. For instance, when $4 D / d_{\text {pore }} \ll \rho_{1,2}$ the relaxation is diffusion-limited (Fig. 4a), and Eq. (39) simplifies to:

$\frac{1}{T_{1,2}}=\frac{1}{T_{1,2 \text { bulk }}}+\frac{8 \alpha D}{d_{\text {pore }}^{2}}$

On the other hand, when $4 D / d_{\text {pore }} \gg \rho_{1,2}$, the relaxation is surface-limited (Fig. 4b), and one has:

$\frac{1}{T_{1,2}}=\frac{1}{T_{1,2 b u l k}}+\frac{2 \alpha \rho_{1,2}}{d_{\text {pore }}}$

These expressions thus directly relate the relaxation times $T_{1}$ or $T_{2}$ to either $d_{\text {pore }}^{2}$ or $d_{\text {pore }}$, depending on whether diffusion-limited or surface-limited relaxation predominates, respectively. Eqs. (40a) and (40b) thus depend very differently on pore-size, which allows the limiting relaxation processes occurring in pores to be identified. Godefroy et al. have shown by low field NMR relaxation at $2.2 \mathrm{MHz}$ that changing the quantity of surface paramagnetic impurities leads to strikingly different pore-size dependences of $T_{1}$ and $T_{2}$ for liquids in pores [47]. They used a series of samples containing individually sorted and packed non-porous $\mathrm{SiC}$ grains between 8 and $150 \mu \mathrm{m}$ in size to show how $T_{1}$ and $T_{2}$ data of liquids changed between the surface-limited and diffusion models of relaxation as a function of pore size of oil-bearing rocks for which $25 \%$ of the surface is covered by $\mathrm{SiO}_{2}$. Two sets of samples were prepared, one with and one without removal of surface paramagnetic impurities by cleaning with hydrochloric acid. After cleaning, Electron Spin Resonance (ESR) showed the nature and remaining quantity of chemically bonded $\mathrm{Fe}^{3+}$ paramagnetic ions. $T_{1}$ and $T_{2}$ relaxation times of water fully saturating these samples were measured at $2.2 \mathrm{MHz}$ and $34^{\circ} \mathrm{C}$. For the uncleaned porous media, which contain a high surface density of paramagnetic impurities (Fig. 4a), the relaxation times varied as the square of the pore diameter $d_{\text {pore }}$, agreeing well with predictions for diffusion-limited relaxation (Eq. (40a) with $\alpha=3$ for a quasi-spherical pores) and providing, from the $T_{1}$ data, an estimate of translational diffusion in pores $D=2.3 \times 10^{-5} \mathrm{~cm}^{2} / \mathrm{s}$ at $34{ }^{\circ} \mathrm{C}$. For the cleaned porous media, which contain a low surface density of paramagnetic impurities, the results show a linear pore-size dependence of $T_{1 \text { corr }}$ and $T_{2 \text { corr }}$, (Fig. 4b) (here $1 / T_{1,2 \text { corr }}=1 / T_{1,2}-1 / T_{1,2 b u l k}$ ), agreeing well with predictions for surface-limited relaxation (Eq. (40b)). Estimates from the slopes of the linear fits of Fig. $4 \mathrm{~b}$ of the longitudinal and transverse surface relaxivity parameters give values $\rho_{1}=0.94 \mu \mathrm{m} / \mathrm{s}$ and $\rho_{2}=3.4 \mu \mathrm{m} / \mathrm{s}$; these values are typical of those for natural rock samples, thus demonstrating that it is surface-limited relaxation processes that are relevant in these widely encountered porous systems. 


\section{Striking differences between NMRD features of water and oil in bulk and confinement}

Fig. 5 shows proton nuclear magnetic relaxation dispersion (NMRD) profiles of water and oil (dodecane) embedded in a carbonate reservoir rock as a function of temperature [47]. In this example, the sample porosity is $24 \%$ with a water permeability of $1.5 \mathrm{mD}$ (millidarcy) (1 Darcy is $10^{-12} \mathrm{~m}^{2}$ ); this rock sample is a non-strongly water-wet material where the surface interaction is weak. For water, the leveling off that appears at low frequency at $65{ }^{\circ} \mathrm{C}$ is a good indication of a molecular desorption of the surface proton species. From mercury porosimetry, the pore throat radius distribution is narrow and peaks at $0.5 \mu \mathrm{m}$. ESR experiments have shown the presence of $\mathrm{Mn}^{2+}$ paramagnetic ions. One notes that in the bulk phase the relaxation rate $1 / T_{1}$ for oil is greater than for water; this is a consequence only of their different correlation times and molecular sizes. In contrast, in rocks the relaxation rate of water is greater than that of oil (Fig. 5). As described above, in pores the surface contribution dominates the relaxation at low frequency. The proton spin-relaxation is due to the modulation of dipole-dipole interactions between the mobile proton species $\left({ }^{1} \mathrm{H}\right.$ as $I$ spin) and the fixed paramagnetic spins $\left(\mathrm{Mn}^{2+}\right.$ as $S$ spin $)$ caused by translational surface diffusion. The bi-logarithmic frequency dependence observed in Fig. 5 reflects the back and forward molecular motions of molecules close to the pore surface where the paramagnetic ions are situated.

\section{NMRD of longitudinal relaxation rate in calibrated micropores}

\subsection{Theoretical background}

According to the biphasic fast exchange model (Eq. (38)), in the fast diffusion limit the overall proton relaxation rate $1 / T_{1}$ is a linear combination of a bulk $1 / T_{1, \text { bulk }}$ and a surface relaxation rate $1 / T_{1, \text { surf }}$ :

$\frac{1}{T_{1}\left(\omega_{I}\right)}=\frac{1}{T_{1, \text { bulk }}}+\frac{1}{T_{1, \text { surf }}\left(\omega_{I}\right)}$

The bulk relaxation term, $1 / T_{1, \text { bulk, }}$, is independent of the Larmor frequency $\omega_{I} / 2 \pi$ in the range that is relevant here [43]. The surface

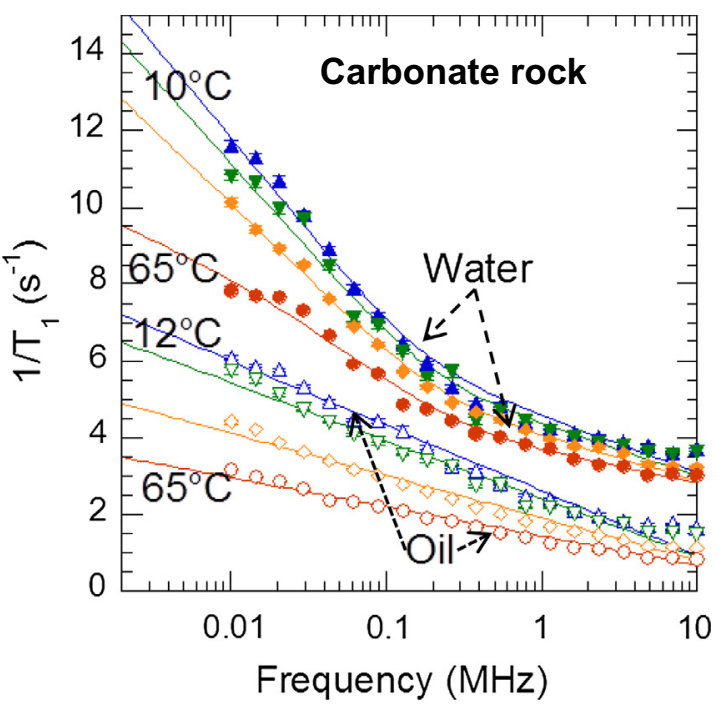

Fig. 5. Frequency dependencies of ${ }^{1} \mathrm{H}$ spin-lattice relaxation rates of water or oil embedded in a carbonate reservoir rock from $10^{\circ} \mathrm{C}$ (top) to $65^{\circ} \mathrm{C}$ (bottom). The data come from Refs. $[25,26]$. The continuous lines are obtained from best fits obtained with Eq. (55). relaxation rate $1 / T_{1, \text { surf }}$ is the sum of the contribution $1 / T_{1,2 D}$ of the proton species ( $I$ ) diffusing laterally (two-dimensionally) in the proximity of a fixed paramagnetic species $(S)$ and the contribution $1 / T_{1, \text { param }}$ of the proton species linked to the first coordination sphere of paramagnetic centers [15]:

$\frac{1}{T_{1, \text { surf }}\left(\omega_{I}\right)}=\frac{N_{\text {Surface }}}{N} \frac{1}{T_{1,2 D}\left(\omega_{I}\right)}+\frac{N_{\text {param }}}{N} \frac{1}{T_{1, \text { param }}\left(\omega_{I}\right)}$

The two surface relaxation terms in $1 / T_{1, \text { surf }}$ are highly sensitive to local physical and chemical effects at the pore surface, resulting in different frequency behaviours in the two cases. The inclusion of the nuclear paramagnetic term is critical because it extends the theory to make the NMRD of protic and aprotic liquids different from one another $[15,16,20,21]$. The terms protic and aprotic are defined according to whether protons in the liquids under study respectively can or cannot undergo facile chemical exchange; this exchangeable character is a distinct property from the polarity of such liquids, and is closely linked to their ability to participate in hydrogen bonds. Only a protic liquid can act as a hydrogen bond donor. In contrast, although aprotic liquids may be highly polar, as are for instance acetone, DMSO, DMF or acetonitrile, they cannot act as hydrogen bond donors as they contain only nonexchangeable protons. The consideration of exchangeability of protons at pore surfaces clarifies the weak and strong adsorption limits introduced previously by Kimmich et al. [5,67]. The calculation of these two contributions to relaxation in the local layer geometry at proximity of the pore surface (Fig. 6) is outlined below. In Eq. (41b), $N_{\text {Surface }} / N=\lambda S_{P} \rho_{\text {liquid }}$ is the ratio between, on the one hand, the number $N_{\text {Surface }}$ of liquid molecules diffusing within a thin transient layer $\lambda$ of the order of a few molecular sizes [66] close to the pore surface, and, on the other, the bulk liquid population $N$. The term $S_{p}$ is the true specific surface area of the sample, and $\rho_{\text {liquid }}$ is the density of the liquid. $\frac{N_{\text {param }}}{N}=\left(\frac{N_{\text {param }}}{N_{S}}\right)\left(\frac{N_{S}}{N}\right) \ll \frac{N_{S}}{N}$ is the ratio between the number of liquid molecules transiently bonded to the paramagnetic sites at the surface and the number in the bulk. The paramagnetic sites located at the pore surface have a ligand field which can trap a moving proton species defined as a protic liquid wetting the surface, but, in contrast, does not trap a moving proton species defined as an aprotic liquid. These different chemical behaviours result in different features in the NMRD data that can be characterized by the terms $\frac{1}{T_{1,2 D}\left(\omega_{1}\right)}$ or $\frac{1}{T_{1, \text { param }}\left(\omega_{1}\right)}$ in Eq. (41b).

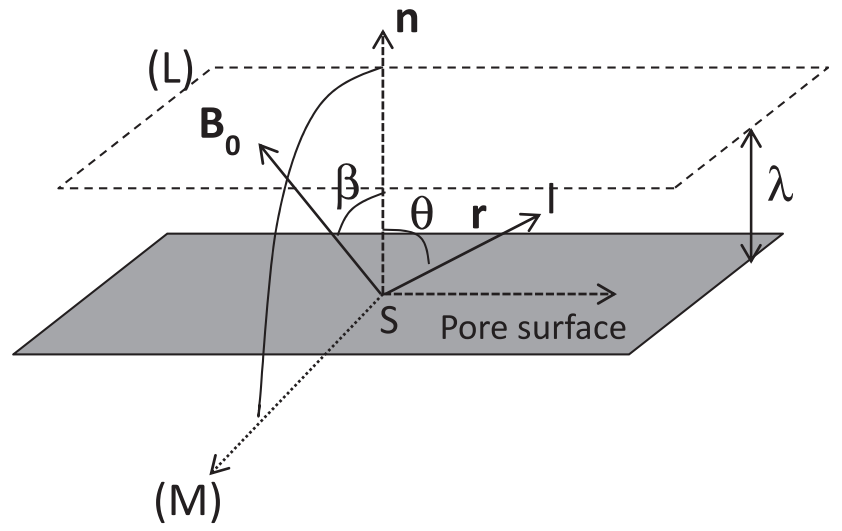

Fig. 6. Schematic diagram showing the lamellar surface diffusion spin-relaxation model and its associated parameters, $S$ and $I$ represent the fixed paramagnetic species and the mobile proton nuclei. $(L)$ and $(M)$ represent the laboratory and lamellar frames, respectively. $\boldsymbol{n}$ is the normal direction to the pore surface and $\boldsymbol{B}_{0}$ is the constant magnetic field. $\lambda$ represents the thickness of the thin liquid layer influenced by the surface relaxation (see the model in Section 6.2). 


\subsection{Aprotic liquids}

Consider first the case of $1 / T_{1,2 D}(\omega)$, corresponding to a protonbearing, aprotic liquid diffusing close to the solid-liquid interface in a porous medium that contains on its surface a very small quantity of fixed paramagnetic species of spins, $S$, uniformly distributed with a surface density $\sigma_{s}$. For an aprotic liquid, the term $\frac{1}{T_{1 \text { param }}\left(\omega_{l}\right)}$ disappears in Eq. (41b). As the magnetic moment of the paramagnetic species is large $\left(\gamma_{S}=659 \gamma_{I}\right)$ compared to that of the proton $\gamma_{\text {I }}$, the only significant nuclear spin relaxation mechanism for the diffusing proton spins, $I$, is that of intermolecular dipole-dipole relaxation induced by the fixed $S$ spins and modulated by the two-dimensional (2D) translational diffusion of the mobile I spins, while they are in close proximity to the paramagnetic sinks fixed on the pore surfaces. In this case, the nuclear spin relaxation times are given formally by the general heteronuclear expression [43]:

$$
\begin{aligned}
\frac{1}{T_{1,2 D}\left(\omega_{I}\right)}= & \left.\frac{\mu_{0}}{4 \pi}\right)^{2} \frac{2}{3}\left(\gamma_{I} \gamma_{S} \hbar\right)^{2} S(S+1) \\
& \times\left[\frac{1}{3} J_{L}^{(0)}\left(\omega_{I}-\omega_{S}\right)+J_{L}^{(1)}\left(\omega_{I}\right)+2 J_{L}^{(2)}\left(\omega_{I}+\omega_{S}\right)\right],
\end{aligned}
$$

where $J_{L}{ }^{(m)}(\omega)(m \in\{-2,2\})$ are the spectral densities in the Laboratory frame ( $L$ associated to the constant direction of the magnetic field $\boldsymbol{B}_{0}$ (see Fig. 6)). As for a heteronuclear relaxation process, they are expressed at the Larmor frequencies of the electron $\omega_{S}$ and proton $\omega_{I}$, these being related by $\omega_{S}=658 \omega_{I}$ and defined as the exponential Fourier transforms:

$J_{L}^{(m)}(\omega)=\int_{-\infty}^{+\infty} G_{L}^{(m)}(\tau) e^{-i \omega \tau} d \tau$

of the stationary pairwise dipolar correlation functions $G_{L}{ }^{(m)}(\tau)$ $(m \in\{-2,2\})$ given in the laboratory frame $L$ by:

$G_{L}^{(m)}(\tau)=\left\langle F_{L}^{(-m)}(t) F_{L}^{(-m)} *(t+\tau)\right\rangle$.

In a semi-classical treatment of random dipolar fluctuations, $G_{L}^{(m)}(\tau)$ describes the persistence of the autocorrelation function of the dipole-dipole interactions $F_{L}{ }^{(m)}(t) \sim Y_{2}{ }^{m}[\Omega(t)] / r_{I S}{ }^{3}(t)$ between spins $I$ and $S$ that are modulated during a short time interval $\tau$ by translational diffusion of spins $I$ at distance $r_{I S}$ from a paramagnetic spin $S$ fixed on the pore surface. $Y_{2}{ }^{m}[\Omega(t)]$ represents the 2nd rank spherical harmonics $(m \in\{-2,2\})$ that depend on the orientation angles $\Omega(t)$ of $\vec{r}_{I S}(\tau)$. In Eq. (43b), the notation $\langle\ldots\rangle$ represents an ensemble average over all the positions of the $I$ spins. This ensemble average is generally expressed as an integral average over a normalized diffusive propagator $P\left(\vec{r}_{0}, \vec{r}, \tau\right)$ :

$G_{L}^{(m)}(\tau)=\int d \vec{r}_{0} p\left(\vec{r}_{0}\right) F_{L}^{(-m)}(0) \int d \vec{r} P\left(\vec{r}_{0}, \vec{r}, \tau\right) F_{L}^{(-m)} *(\tau)$

Here $P\left(\vec{r}_{0}, \vec{r}, \tau\right)$ is a solution of a diffusive equation with initial and boundary conditions and $p\left(\vec{t}_{0}\right)=\sigma_{S} / \lambda$ represents the equilibrium and uniform density of spin pairs $I-S$ at equilibrium.

In the following, an outline is given of the five main steps allowing asymptotic solutions of Eq. (44) at long times (low frequency).

(i) The well-known rotation properties of spherical harmonics $Y_{2}{ }^{m}[\Omega(t)]$ in different bases make the calculations of Eq. (44) easier to handle in the lamellar frame $(M)$ of Fig. 6 before transforming back to the laboratory frame $(L)$ associated with the constant direction of the magnetic field $\boldsymbol{B}_{0}$ :

$G_{L}^{(m)}(\tau)=\sum_{m^{\prime}=-2}^{+2}\left|d_{-m^{\prime}, m}^{(2)}(\beta)\right|^{2} G_{M}^{\left(m^{\prime}\right)}(\tau)$

Here the $d_{-m, m^{\prime}}^{(2)}(\beta)$ are the well-known Wigner functions [68] allowing expression of the dipolar interaction $F_{L}^{(m)}(t)$ as it comes through a succession of coordinate rotations in the different frames $(M) \rightarrow(L)$ while $G_{M}^{\left(m^{\prime}\right)}(\tau)$ with $\left\{m^{\prime} \in(-2,+2)\right\}$ are the pairwise dipolar correlation functions in the lamellar frame $(M)$. These latter functions are given by an integral average over the propagator of diffusion in the lamellar frame (M), formally similar to Eq. (44).

(ii) In the anisotropic dynamic model presented in Fig. 6, there is an unbounded and isotropic diffusion perpendicular to the normal axis $\boldsymbol{n}$, and a bounded diffusion along this axis. According to this model, the normalized conditional probability $P$ in the planar lamellar frame $(M)$ is thus defined as a product of a bounded $P_{/ /}$ and an unbounded $P_{\perp}$ propagator of diffusion:

$P\left(\rho, z, \tau \mid \rho_{0}, z_{0}, 0\right)=P_{\perp}\left(\rho, \tau \mid \rho_{0}, 0\right) P_{/ /}\left(z, \tau \mid z_{0}, 0\right)$.

$P_{/ /}$is given by the solution of a 1D-diffusive equation with no flux out of the layer, while $P_{\perp}$ is expressed by its Fourier transform in the reciprocal $k$ space [69]:

$$
\begin{aligned}
P_{/ /}\left(z, \tau \mid z_{0} 0\right) \approx & \frac{1}{\lambda}\left[1+2 \cos (\pi z / \lambda) \cos \left(\pi z_{0} / \lambda\right) \exp \left(-D_{I / /} \tau / \lambda^{2}\right)+\cdots\right] \\
P_{\perp}\left(\rho, \tau \mid \rho_{0}, 0\right)= & \frac{1}{2 \pi} \sum_{m=-\infty}^{+\infty} \int_{0}^{\infty} d k k \exp \left(-k^{2} D_{I \perp} \tau\right) J_{m}(k \rho) J_{m}\left(k \rho_{0}\right) \\
& \times \exp \left[i m\left(\phi_{0}-\phi\right)\right]
\end{aligned}
$$

Here $D_{I / /}$ and $D_{I \perp}$ are the translational diffusion coefficients of spins $I$ in direction parallel and perpendicular to $\boldsymbol{n}$ and the $J_{m}(k \rho)$ are cylindrical Bessel functions of integer order $m$.

(iii) At long times $\tau \rightarrow \infty$ (or low frequency), $P_{/ /}$simplifies to the inverse of the "volume" $(1 / \lambda)$ visited by the fast diffusing protons, which is set by the boundary of zero flux along the $\boldsymbol{n}$ direction on the two limits of the layer of thickness $\lambda$. For $P_{\perp}$, only the long wavelength transverse diffusing modes dominate $(k \rightarrow 0)$ for times $\tau$ much longer than the transverse diffusion correlation time: $\tau_{m}=\delta^{2} /\left(4 D_{I \perp}\right)$, where $\delta$ is the average molecular size of the $I$-spin bearing molecule. The propagator for diffusion in the lamellar frame $P\left(\rho, z, \tau \mid \rho_{0}, z_{0}, 0\right)=P_{\perp}\left(\rho, \tau \mid \rho_{0}, 0\right) P_{/ /}\left(z, \tau \mid z_{0}, 0\right)$, is then substituted in the integral definition of $G_{M}^{\left(m^{\prime}\right)}(\tau)$ with $\left\{m^{\prime} \in(-2,+2)\right\}$, giving at long times $\left(\tau \gg \tau_{m}\right)$ :

$G_{M}^{\left(m^{\prime}\right)}(\tau)=\frac{2 \pi \sigma_{S}}{\lambda^{2}} \int_{0}^{\infty} d k k \exp \left(-k^{2} D_{I \perp} \tau\right)\left|\int d z \int d \rho \rho J_{m}(k \rho) f_{2}^{\left(m^{\prime}\right)}(\rho, z)\right|^{2}$

where $f_{2}^{(m)} \sim 1 / r_{I S}{ }^{3}$ are the dipolar terms expressed in the cylindrical frame $(M)$ (Fig. 6). It is helpful to introduce the distance of minimal approach $\delta^{\prime}$ between spins $I$ and $S$ at pore surface. Typically $\delta^{\prime}$ is comparable to the molecular diameter $\delta$; however, a parameter $x$ $=\delta / \delta^{\prime}$ has been introduced for taking into account a variable distance of minimal approach in proximity of the spin $S$. The integral calculation of Eq. (48) is separated into two spatial domains: $M_{1}=\left\{\delta^{\prime} \leqslant z \leqslant \lambda\right.$ and $\left.0 \leqslant \rho<\infty\right\}$ and $M_{2}=\left\{0 \leqslant z \leqslant \delta^{\prime}\right.$ and $\delta^{\prime} \leqslant \rho$ $7<\infty\}$ in the layer frame $M$ (Fig. 6). The calculations of Eq. (48) simplify for times $\tau$ much longer than the two-dimensional correlation time $\tau_{m}$, which itself depends on the molecular size $\delta$ and the lateral diffusion coefficient $D_{I \perp}$ as defined above. This approximation allows consideration only of the dominant term coming from the long wavelength two-dimensional transverse modes $k \delta^{\prime} \rightarrow 0$ when $\tau \gg \tau_{\mathrm{m}}$ in the exponential part $\exp \left[-\left(k \delta^{\prime}\right)^{2} x^{2} \tau /\left(4 \tau_{m}\right)\right]$ of Eq. (48). After some calculations, the leading terms come from the two-dimensional part of $G_{M}^{(0)}(\tau)$ integrated in the $M_{2}$ domain:

$G_{M}^{(0)}(\tau) \approx \frac{3 \pi \sigma_{S}}{4 \lambda^{2} \delta^{\prime 2}} \frac{1}{x^{2}}\left(\frac{\tau_{m}}{\tau}\right)+\vartheta\left[\left(\tau_{m} / \tau\right)^{3 / 2}\right]$,

$G_{M}^{(1)}(\tau)$ and $G_{M}^{(2)}(\tau) \approx \vartheta\left[\left(\tau_{m} / \tau\right)^{2}\right]$ 
So, at long times and in the low frequency range studied, only $G_{M}^{(0)}(\tau)$ matters [70,71]. It is physically interesting to represent $G_{M}^{(0)}(\tau)$ as:

$G_{M}^{(0)}(\tau) \approx \frac{3 \pi^{2}}{4}\left(\frac{\sigma_{S}}{\lambda}\right)\left(\frac{1}{V(\tau)}\right)$

where the volume of the cylinder $V(\tau)$ explored by the quasi twodimensional diffusion in a time $\tau$ in the layer frame $(M)$ is introduced:

$V(\tau)=\pi\left(4 D_{I \perp} \tau\right) \lambda$

The time dependence of Eq. (50a) thus becomes quite similar to that given by the prefactor of a Gaussian probability for diffusion. This proves that, at long times, the pairwise dipolar correlation function $G_{M}^{(0)}(\tau)$ is clearly proportional to the probability of a reencounter between spins $I$ and $S$ resulting from two-dimensional diffusion in a given time $\tau$.

(iv) For physical reasons $G_{M}^{(0)}(\tau)$ must also fulfil the three following requirements [43]. (a) At short times, when $\tau \rightarrow 0, G_{M}^{(0)}(\tau)$ must tend to a finite constant $C$, such as $G_{M}^{(0)}(0)=\int_{-\infty}^{\infty} J_{M}^{(0)}(\omega) d \omega=C$, where $J_{M}^{(0)}(\omega)$ is the spectral density given by the Fourier transform of $G_{M}^{(0)}(\tau)$. (b) Eq. (49a) shows that, at long times, $G_{M}^{(0)}(\tau)$ must behave as a power law $\propto 1 / \tau$ characteristic of a dipolar intermolecular relaxation process by two-dimensional translational diffusion. However, there must always be a finite time of residence $\tau_{s}$ at a pore surface that creates an exponential cut-off for the dipolar correlations: $G_{M}^{(0)}(\tau) \propto(C / \tau) \exp \left(-|\tau| / \tau_{S}\right)$. (c) The form of $G_{M}^{(0)}(\tau)$ on the time scale of surface molecular diffusion events, $0 \leq \tau \sim \tau_{m} \ll \tau_{s}$, must be characterized by the correlation time for the surface diffusion events. In combination, all these requirements lead to the following expression of the correlation function $G_{M}^{(0)}(\tau)$ which is, as expected, always positive:

$$
\begin{aligned}
G_{M}^{(0)}(\tau) & =\frac{C}{\tau}\left[\exp \left(-\left|\frac{\tau}{\tau_{S}}\right|\right)-\exp \left(-\left|\frac{\tau}{\tau_{m}}\right|\right)\right] /\left(1 / \tau_{m}-1 / \tau_{S}\right) \\
& \approx C \frac{\tau_{m}}{\tau}\left[\exp \left(-\left|\frac{\tau}{\tau_{S}}\right|\right)-\exp \left(-\left|\frac{\tau}{\tau_{m}}\right|\right)\right] \text { when } \tau_{\mathrm{m}} \ll \tau_{S}
\end{aligned}
$$

Straightforward calculations give $C=3 \pi \sigma_{S} /\left(4 x^{2} \lambda^{2} \delta^{\prime 2}\right)$. The spectral density function is thus given by the exponential Fourier transform of Eq. (51) [25]:
$J_{M}^{(0)}(\omega)=\frac{3 \pi \sigma_{S}}{4 x^{2} \lambda^{2} \delta^{\prime 2}} \tau_{m} \ln \left[\frac{1+\omega^{2} \tau_{m}^{2}}{\left(\tau_{m} / \tau_{S}\right)^{2}+\omega^{2} \tau_{m}^{2}}\right]$.

(v) Last, calculating a powder average of $J_{M}^{(m)}(\omega)$ over all the orientations $\beta$ of the $(M)$ frame relative to the constant direction of $\boldsymbol{B}_{0}$ gives the average spectral densities:

$\left\langle J_{L}^{(0)}(\omega)\right\rangle=\left\langle J_{L}^{(1)}(\omega)\right\rangle=\left\langle J_{L}^{(2)}(\omega)\right\rangle \approx \frac{1}{5} J_{M}^{(0)}(\omega)$.

Substitution of Eq. (52) into Eq. (53), gives the required powder average of the spectral density in the laboratory frame $(L)$ :

$$
\begin{aligned}
\left\langle J_{L}^{(m)}(\omega)\right\rangle & =\int_{-\infty}^{+\infty}\left\langle G_{L}^{(m)}(\tau)\right\rangle e^{-i \omega \tau} d \tau \\
& =\frac{3 \pi \sigma_{S}}{20 x^{2} \lambda^{2} \delta^{\prime 2}} \tau_{m} \ln \left[\frac{1+\omega^{2} \tau_{m}^{2}}{\left(\tau_{m} / \tau_{S}\right)^{2}+\omega^{2} \tau_{m}^{2}}\right] .
\end{aligned}
$$

After substitution of Eq. (54) into Eq. (42), one obtains the following bi-logarithmic frequency dependence of the overall spin-lattice relaxation rate $1 / T_{1}(\omega)$ of the $2 \mathrm{D}$ diffusion of an aprotic liquid in the proximity of the pore surface [72]:

$$
\begin{aligned}
\frac{1}{T_{1}\left(\omega_{I}\right)}= & \frac{1}{T_{1, \text { bulk }}}+\left(\frac{\mu_{0}}{4 \pi}\right)^{2} \frac{\pi \lambda S_{P} \rho_{\text {liquid }} \sigma_{S}\left(\gamma_{I} \gamma_{S} \hbar\right)^{2} S(S+1)}{15 \lambda^{2} \delta^{\prime 2} x^{2}} \\
& \tau_{m}\left[3 \ln \left(\frac{1+\omega_{I}^{2} \tau_{m}^{2}}{\tau_{m}^{2} / \tau_{S}^{2}+\omega_{I}^{2} \tau_{m}^{2}}\right)+7 \ln \left(\frac{1+\omega_{S}^{2} \tau_{m}^{2}}{\tau_{m}^{2} / \tau_{S}^{2}+\omega_{S}^{2} \tau_{m}^{2}}\right)\right]
\end{aligned}
$$

In Eq. (55), as defined previously, $x=\delta / \delta^{\prime}$ is a parameter introduced to take into account the variable distance $\delta^{\prime}$ of minimal approach between $I$ and $S$ spins relative to the diameter $\delta$ of molecules of the moving liquid under study. The surface concentration of spins of $\mathrm{Fe}^{3+}$ or $\mathrm{Mn}^{2+}$ paramagnetic ions (for both of which $S=$ $5 / 2)$ is given by $\sigma_{S}=\left(\eta_{S} \rho_{\text {solid }} \xi\right)$, where $\eta_{S}$ is the volume concentration of paramagnetic ions measured by ESR, $\xi \sim 0.5 \mathrm{~nm}$ is a thin layer of paramagnetic ions corresponding for instance to the lattice constant of the solid structure in the proximity of the pore surface and $\rho_{\text {solid }}$ is the density of the solid material [27]. Eq. (55) has a bi-logarithmic frequency dependence (on the proton Larmor frequency $\omega_{I}$ and electronic frequency $\omega_{S}=659 \omega_{I}$ ) due to the numerous 2D molecular re-encounters between spins $I$ and $S$ occurring within the thin transient layer $\lambda$. Eq. (55) also contains two correlation times: the translational correlation time, $\tau_{m}$, associated with individual jumps while the molecule is close to the surface, and also the surface residence time, $\tau_{s}$, which is limited by molecular
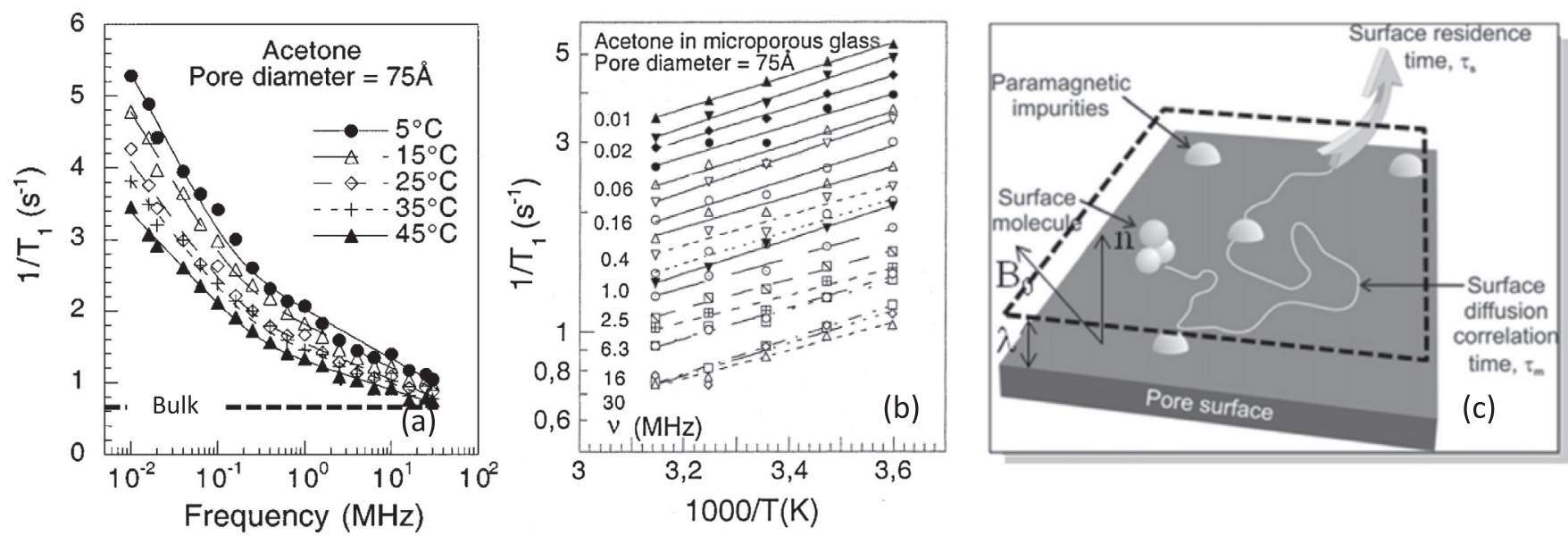

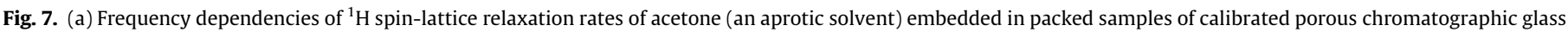

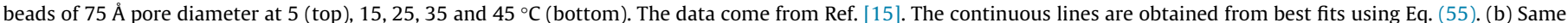

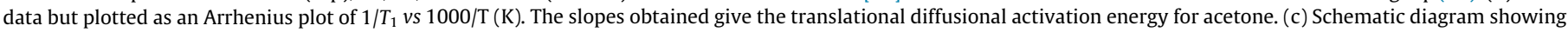
the surface diffusion spin-relaxation model described in Section 6.2. 
desorption from the thin surface layer $\lambda$ and describes how long the proton species I and the $\mathrm{Fe}^{3+}$ or $\mathrm{Mn}^{2+}$ ion $S$ stay correlated; this depends both on the strength of the hydrogen bonds and on the re-occurrence of first neighbour interactions induced by the fluid confinement in pores.

Fig. 7a shows $1 / T_{1}$ proton-NMRD profiles of acetone, which is aprotic and polar, for suspensions of controlled pore chromatographic glasses with mean pore diameters of $75 \AA$ and specific area of $140 \mathrm{~m}^{2} / \mathrm{g}$; results are shown from 0.01 to $30 \mathrm{MHz}$ over a range of temperature from 5 to $45^{\circ} \mathrm{C}$ [15]. Similar profiles were obtained with other aprotic liquids such as acetonitrile, N,Ndimethylformamide (DMF) and dimethylsulfoxide (DMSO) [15]. A common feature of these profiles is the bi-logarithmic dependence on Larmor frequency, in agreement with Eq. (55). The iron content of these $75 \AA$ pore glasses, checked by ESR and analytical chemistry measurements, is $45 \mathrm{ppm}$, which is sufficient to provide the dominant relaxation path for the proton spins at low magnetic-field strengths. Taking the specific area and assuming a homogeneous volume density of paramagnetic species, the surface density $\sigma_{S}$ of paramagnetic centers in the $75 \AA$ glass is $3.46 \times 10^{11} \mathrm{Fe}^{3+} \mathrm{cm}^{-2}$, which would imply an average distance between paramagnetic centers in the order of $170 \AA$ (corresponding to $1 / \sqrt{ } \sigma_{s}$ ). These figures provide an estimate of the persistence length of the two dimensional character that is sensed by the magnetic relaxation of the proton spins induced by the paramagnetic centers.

The good fits obtained with Eq. (55) at different temperatures for the NMRD data of Fig. 7a proves the validity of the model described here and originally proposed in Ref. [15]. The observed $10 / 3$ ratio between the slopes at low and high frequencies is a key feature of an intermolecular dipole-dipole relaxation process

Table 1

Relaxation parameters in calibrated porous systems [15].

\begin{tabular}{llll}
\hline \multirow{2}{*}{ Calibrated porous systems } & \multicolumn{2}{l}{$\mathrm{d}_{\text {pore }}=75 \AA$} \\
Liquids & \multicolumn{2}{l}{$\sigma_{\mathrm{s}}=3.46 \times 10^{11} \mathrm{Fe}^{3+} / \mathrm{cm}^{2}$} \\
\cline { 2 - 4 }$\delta(\AA)$ & $\delta^{\prime}(\AA)$ & $\mathrm{D}_{\perp \perp}\left(10^{-5} \mathrm{~cm}^{2} / \mathrm{s}\right)$ \\
\hline Acetone & 6.1 & 3.7 & 0.14 \\
Acetonitrile & 6.2 & 3.6 & 0.16 \\
DMF & 7.5 & 4.4 & 0.03 \\
DMSO & 7.1 & 6.0 & 0.04 \\
\hline
\end{tabular}

[43]. This bi-logarithmic NMRD profile is also typical of the theoretically predicted 2-dimensional diffusion-relaxation of a liquid in the proximity of the pore surface $[70,71]$. The fact that $1 / T_{1}$ decreases when the temperature increases is also typical of relaxation mediated by diffusive transport. The temperature dependencies of $1 / T_{1}$ follow an Arrhenius plot for every frequency (Fig. 7b) whose individual slope gives an activation energy that is consistent with the value expected for acetone. It is important to note that Eq. (55) was derived without explicit inclusion of the electron-spin relaxation rate, $1 / T_{1, \text { param }}$, as a potential source of important fluctuations in the electron-nuclear coupling. If the electron-spin relaxation time were sufficiently short that it competed with the translational diffusion times, then the low-field portion of the relaxation dispersion would be independent of field strength [73], which is not consistent with observation. Thus the neglect of the electron-spin relaxation in Eq. (42) is supported by the experimental result. Different solvent molecules can be approximated by spheres of different diameters, $\delta$, using a molecular modelling program to estimate the smallest sphere into which a given molecule will fit (the program insight2 [74] was used in the case of the work described in Ref. [15]). This procedure gave values for $\delta$ of $6.12 \AA$, $6.18 \AA$, $7.50 \AA$ and $7.06 \AA$ for acetone, acetonitrile, DMF and DMSO, respectively (Table 1 ). There remain only two adjustable parameters: the distance of minimal approach between the $I$ and $S$ spins, $\delta^{\prime}$, and the translational diffusion coefficient, $D_{I \perp}$, for the proton bearing molecule. Moreover, the values of $\delta^{\prime}$ stay constrained by reasonable estimates for the sums of van der Waals radii (perhaps modified in the case that a hydrogen bonding interaction is present with $\mathrm{OH}$ or $\mathrm{FeOH}$ groups at the pore surface). The solid lines through the data in Fig. $7 \mathrm{a}$ and $\mathrm{b}$ were computed with Eq. (55) as best fits to the data, using only $\delta^{\prime}$ and $D_{I \perp}$ as adjustable parameters in the model displayed in Fig. 7c. The fit to the theory is excellent over the range of magnetic fields studied. The parameters appropriate to $298 \mathrm{~K}$ are collected in Table 1 . The values of $\delta^{\prime}$ for the $75 \AA$ glass represent the kinds of number expected for solvent molecules colliding with a completed first coordination sphere environment for the paramagnetic metal center. Similar values have been obtained for metal complexes in solution [75-77]. The values of the diffusion coefficients obtained by this procedure are about an order of magnitude smaller than values for bulk solvent, which is consistent with other measurements of surface translational diffusion [78]. Based on the effects of confinement, it is
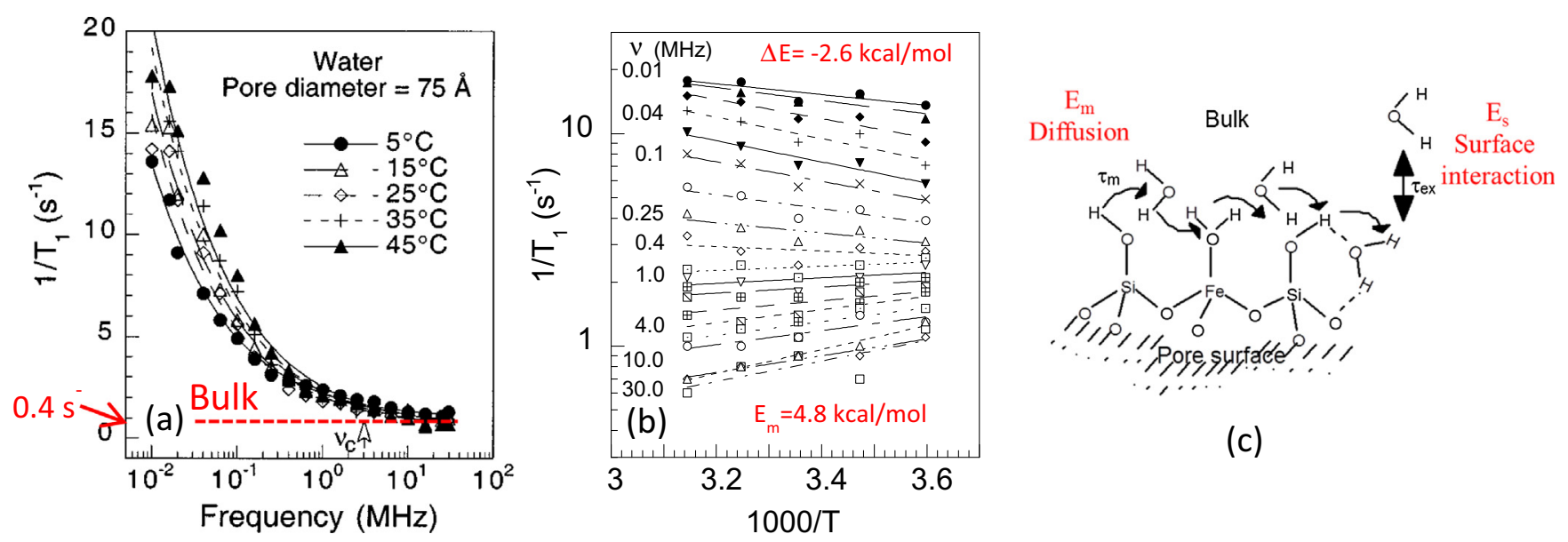

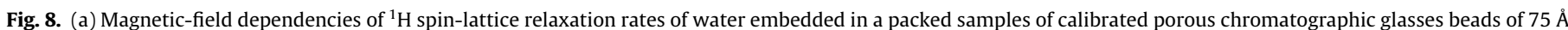

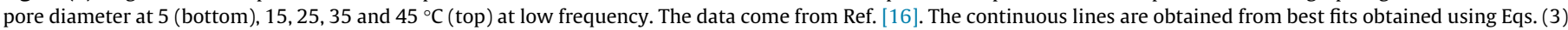

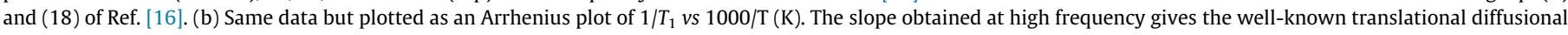

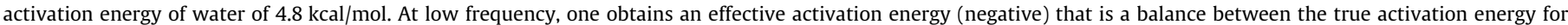

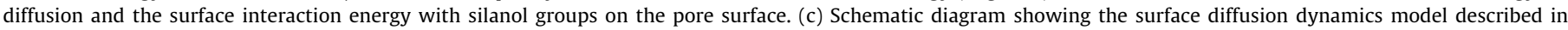
Section 6.3. 
expected that the translational diffusion coefficient should increase with increasing pore size [78,79]. The approximately linear dependence of the diffusion constant on the reciprocal of molecular diameter, $D_{I \perp} v s 1 / \delta$, is consistent with the StokesEinstein law for liquid dynamics in the confined spaces of the pores. This linear pore size dependence for diffusion of a liquid in nano-confinement has been explained recently by complementary experimental and theoretical arguments [80].

\subsection{Protic liquid (water)}

There are remarkable differences between the ${ }^{1} \mathrm{H}$ NMRD profiles of water and those of aprotic solvents when in contact with the same surface-area-calibrated microporous chromatographic glasses described above that contain trace paramagnetic impurities located at or close to the pore surface (Fig. 8a). Surprisingly, the temperature dependences of the ${ }^{1} \mathrm{H}$ NMRD of water and aprotic solvents are opposite at low frequency, despite the fact that they are similar at high frequency (see Figs. $7 b$ and $8 b$ ). Although the calibrated glass does not provide a one-dimensional matrix, the molecular dynamics of water ${ }^{1} \mathrm{H}$ spin relaxation is dominated by long range correlations that make the dynamics behave effectively as though they were 1-dimensional [16].

Water has extensive hydrogen bonding capabilities and may exchange protons with other molecules or surface sites. It may behave as both a Lewis acid or base, and generally coordinates to most metal ions. The water proton spin-lattice relaxation rate is fundamentally different from that of other liquids embedded in glasses in that it shows a power law dependence on the Larmor frequency (Fig. 8a). This dependence results from $I-S$ correlations that persist for much longer times in the surface region than in the bulk, thus leading to a significant increase of $1 / T_{1}$ at low frequency [16]. The nuclear spin-lattice relaxation rate at the pore surface is dominated by dynamic processes that appear to be quasi-1dimensional [16]. An interesting feature is that the temperature dependence is opposite to that usually observed for diffusioninduced relaxation, which is found for aprotic liquids. Such anomalous temperature dependence has been interpreted in terms of a diffusive process at the pore surface that is interrupted by a chemical exchange with the bulk phase (Fig. 8c). The fundamental difference between water and other solvents in these glasses is the spatial extent of the surface explored by the diffusing protons of the liquid. The possibility that water may coordinate directly to paramagnetic relaxation centers is shown to be of minor importance to the observed relaxation dispersion profiles.

The best fits obtained with Eqs. (3) and (18) of Ref. [16] for 1/ $T_{1}\left(\omega_{I}\right)$ are shown as continuous lines in Fig. 8a. The only adjustable parameter here is the effective diffusion $D_{\text {eff }}=0.85 \times 10^{-5} \mathrm{~cm}^{2} / \mathrm{s}$ at $25^{\circ} \mathrm{C}$. This value, for the proton surface diffusion, of the order of one third of the bulk water translational diffusion $D_{w} \sim 2.2 \times$ $10^{-5} \mathrm{~cm}^{2} / \mathrm{s}$, is in very good agreement with neutron scattering studies of single-particle dynamics of water molecules contained in $25 \%$ hydrated micropores of Vycor glass [81], as well as with a molecular dynamics simulation of liquid water on silica surfaces [82]. The Arrhenius plots of $1 / T_{1}$ against temperature for the different frequencies studied (Fig. $8 \mathrm{~b}$ ) present an apparent negative activation energy, $-2.6 \mathrm{kcal} / \mathrm{mol}$, at low frequency and the usual positive activation energy of water $E_{m}=4.8 \mathrm{kcal} / \mathrm{mol}$ at high frequency. The seemingly paradoxical temperature dependence observed for $1 / T_{1}$ at low frequency (Fig. $8 \mathrm{~b}$ ) has been interpreted in terms of an effective surface proton diffusion process (see Fig. 8c) with possible hydrogen bonding with the silanol groups on the pore surface [16]. Essentially, an effective surface diffusion coefficient can be given as a product of a Boltzmann factor $n(T)=n_{0} \exp \left(E_{\text {surf }} / R_{b} T\right)$ and a true diffusion coefficient $D(T)=$ $D_{0} \exp \left(-E_{m} / R_{B} T\right)$. Here $n_{o}$ is the maximum number of molecules in a locally ordered domain; $R_{b}$ is the molar gas constant and $E_{\text {surf }} \sim 12 k_{B} T$ is expected to be of the order of that for a hydrogen bond interaction: i.e. $\sim 7.4 \mathrm{kcal} / \mathrm{mol}$. It thus produces an effective surface diffusion coefficient: $D(T)=D_{0 \text { eff }} \exp \left(-\Delta E / R_{B} T\right)$, where $\Delta E=\left(E_{m}-E_{\text {surf }}\right)$ with $E_{m}<E_{\text {surf. }}$ At low frequency, where the NMRD profile is mainly sensitive to diffusion at the pore surface, the later relation explains the negative value found for $\Delta E=-2.6 \mathrm{kcal} / \mathrm{mol}$, while at high frequency, where the NMRD senses the bulk dynamics, $\Delta E$ tends to the usual bulk diffusional activation energy of water $E_{m}=4.8 \mathrm{kcal} / \mathrm{mol}$ (Fig. $8 \mathrm{~b}$ ). The inversion of the activation energy of $1 / T_{1}$ at low frequency when increasing the temperature, seen in Fig. 8b, gives a proof of the occurrence of surface hydrogen bonding that is typical for a water-wet surface.

\section{Continuous multi-scale NMR relaxation investigation of microstructure evolution of cement-based materials}

\subsection{Cement-based materials}

Concrete is a building material well-known to be composed of cement (usually Portland cement) mixed with aggregates, sand, water and chemical admixtures such as silica fume and adjuvants. After mixing with water and placement, concrete hardens due to hydration processes; the water immediately reacts with the anhydrous cement, which bonds the other components through hydrates and forms a hardened cement paste that is the main ingredient of concrete [83]. The important mechanical properties and durability of concrete are mainly a consequence of the microstructure of hydrated cement paste, which is characterized by different mesoscopic properties such as porosity, specific surface area, distribution and connectivity of pores, as well as tortuosity factor. Questions still exist about the micro-, and especially mesostructure, of such hydrated cement pastes that are highly disordered on multiple scales. Answering these questions is important because such microporous features directly influence mechanical properties as well as transport efficiency of water and aggressive agents. Better control over evolution of the microstructure during hydration and setting remains important if these properties are to be improved in the future.

The following section presents an outline of some non-invasive NMR approaches for investigating the evolution of the microstructure of various cement-based materials under different conditions. The NMRD technique has been used for probing the dynamics of protonated species in the proximity of pore surfaces [20]. Multidimensional NMR relaxation techniques $\left(T_{1}-T_{2}\right.$ and $\left.T_{2}-T_{2}\right)$ have also revealed water exchange between connected micropores $[29,30]$.

The NMRD approach was used by Barberon et al. to study a reactive powder mortar sample prepared by mixing cement, sand, silica fume, water and superplasticizer with a water to cement ratio $w / c$ $=0.38$ [20]. Before addition of water, the specific surface area of the various grains is very low whereas after the dissolutionprecipitation process, calcium silicate hydrate structures ( $\mathrm{CSH}$ ) appear at the surface of the grains leading to a very high surface area that increases continuously during setting. The benefit of exploring relaxation at low frequency in this system is that it allows non-invasive measurement of the typical NMRD dispersion features of $1 / T_{1}$ that are characteristic of the different processes of molecular surface dynamics. In this work, Barberon et al. proposed a model for interpreting the remarkable features of such NMRD profiles, based on solid/liquid cross-relaxation, proton surface diffusion and nuclear paramagnetic relaxation, in the presence of progressive hydration [20]. Fig. 9a shows proton NMRD data obtained for increasing durations of hydration for such a mortar measured at $25^{\circ} \mathrm{C}$ (stabilised temperature); each NMRD profile was acquired sufficiently rapidly ( $\sim 20 \mathrm{~min})$, relative to the hydration kinetics, 

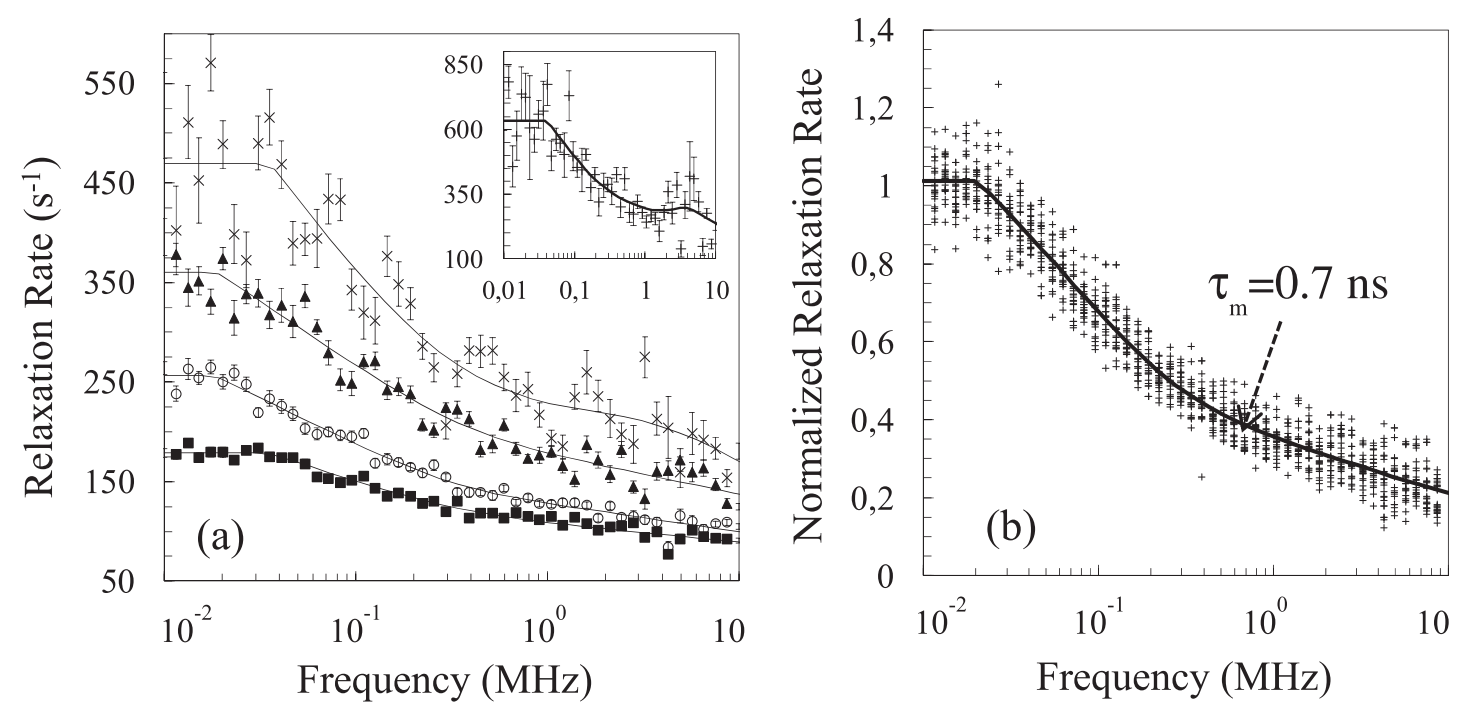

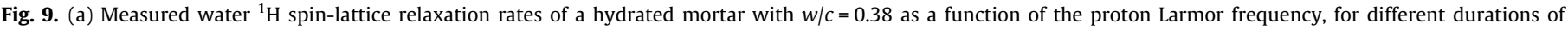

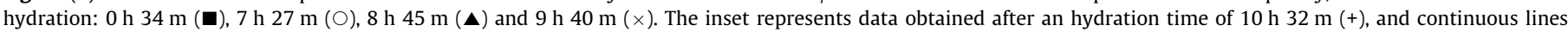

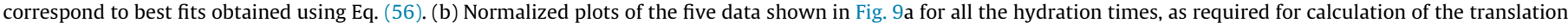
correlation time $\tau_{m} \sim 0.7 \mathrm{~ns}$ (see Table 2 ) for surface dynamics of water at a pore surface in such a hydrated mortar sample with $w / c=0.38$.
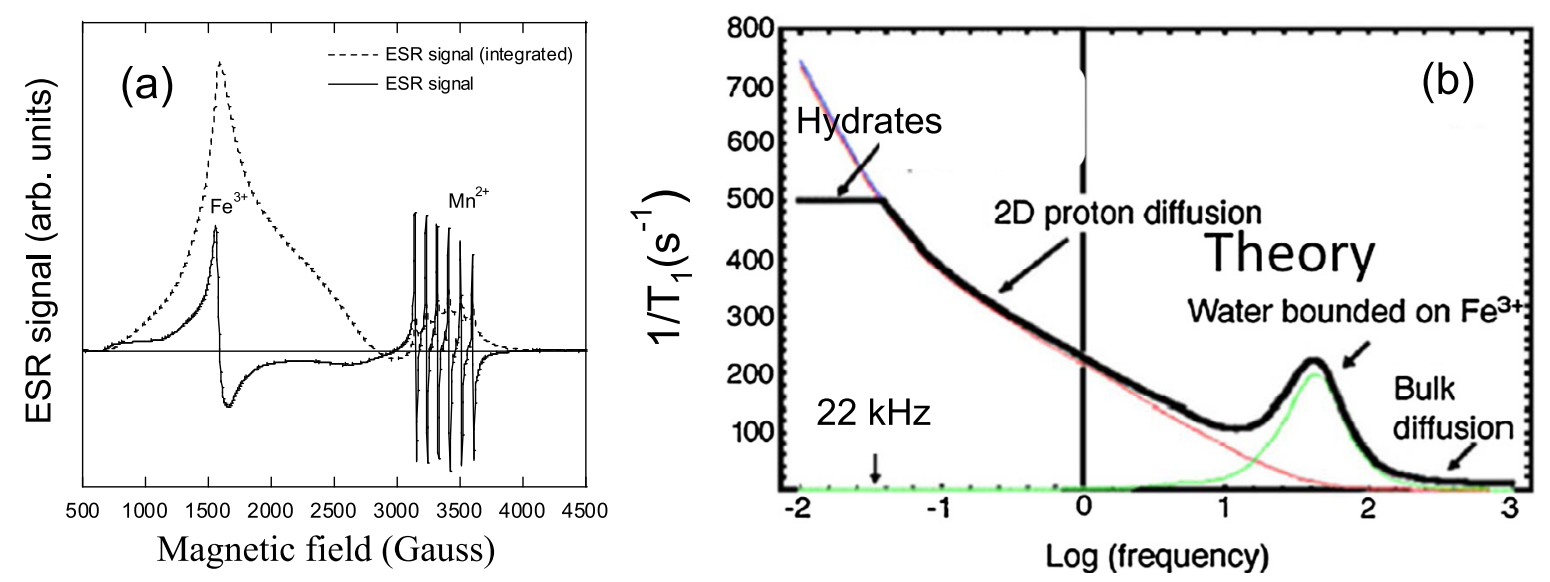

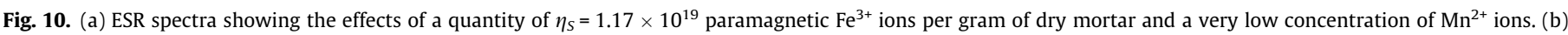

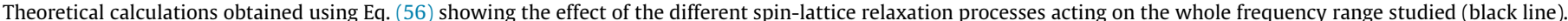

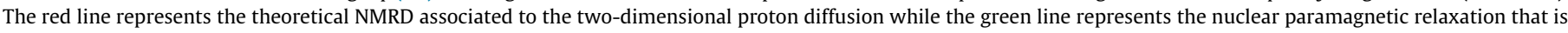
limited to a narrow "bump" in the highest part of the measured frequency range.

that the hydration of the mortar could be followed every thirty minutes during the first twelve hours. These profiles demonstrate three remarkable features of NMRD data for water in hydrated mortars (Fig. 9a). (i) There is a plateau below a cross-over frequency of $\omega_{c}$ $\sim 22 \mathrm{kHz}$. (ii) For frequencies above $\omega_{c}$, for all duration of hydration there is a bi-logarithmic dispersion behaviour, with the two slopes showing a characteristic 10/3 ratio. In Section 6.2 it was shown that this behaviour is indicative of intermolecular dipolar relaxation due to two-dimensional diffusion of water in the proximity of paramagnetic relaxation centers (see Eq. (55)). (iii) Between 3 and $5 \mathrm{MHz}$, relaxation is enhanced at hydration times longer than $10 \mathrm{~h}$ (inset of Fig. 9a). Normalization of the ${ }^{1} H$ NMRD data collected for all durations of hydration was achieved by subtracting the limiting constant value for the bulk relaxation rate at high frequency and then dividing by the value of the respective low frequency plateau (Fig. 9b), and the results of this procedure show that the same nuclear magnetic relaxation process is occurring for all durations of hydration. Quantitative ESR reveals the presence of $\eta_{S}=1.17 \times$ $10^{19}$ paramagnetic $\mathrm{Fe}^{3+}$ ions per gram of dry material and a very small quantity of $\mathrm{Mn}^{2+}$ (Fig. 10a). Assuming a uniform distribution, this leads to a surface density of paramagnetic $\mathrm{Fe}^{3+}$ ions, $\sigma_{S}=\eta_{S}$ $\rho_{\text {solid }} \xi=1.8 \times 10^{12} \mathrm{Fe}^{3+} / \mathrm{cm}^{2}$ to which relaxation of protons of liquids in the saturated porous media are sensitive. Here $\rho_{\text {solid }}=$ $2.5 \mathrm{~g} / \mathrm{cm}^{3}$ is the density of the solid material and $\xi=0.6 \mathrm{~nm}$ is the average inter-layer distance between two $\mathrm{Fe}^{3+}$ ions. Moreover, the Normalization procedure (Fig. 9b) allowed the dynamics of the proton species to be determined by using the following relaxation equation that takes into account diffusion of the water in the proximity of the fixed paramagnetic species $(S)$ as well as the contribution $1 / T_{1 \text {,param }}$ of the proton species linked to the first coordination sphere of paramagnetic centers [20]:

$$
\begin{aligned}
& \frac{1}{T_{1}\left(\left(\omega_{I}, \omega_{S}\right)\right)}=\frac{1}{T_{1, b u l k}}+\left(\frac{\mu_{0}}{4 \pi}\right)^{2} \frac{x \varepsilon}{60} \rho_{w} \sigma_{S} S_{p, N M R}\left(\gamma_{I} \gamma_{S} \hbar\right)^{2} S(S+1) \times \\
& \left\{\frac{\pi}{\varepsilon^{4}} \tau_{m}\left[7 \ln \left(1+\frac{1}{\omega_{S}^{2} \tau_{m}^{2}}\right)+3 \ln \left(1+\frac{1}{\omega_{I}^{2} \tau_{m}^{2}}\right)\right]\right. \\
& \left.\quad+\frac{8 n \varepsilon^{2}}{r_{I S}^{6}} \tau_{c}\left[\frac{7}{1+\omega_{S}^{2} \tau_{c}^{2}}+\frac{3}{1+\omega_{I}^{2} \tau_{c}^{2}}\right]\right\} .
\end{aligned}
$$


Table 2

Dynamical parameters extracted from NMRD profiles on cement-based materials [87].

\begin{tabular}{|c|c|c|c|}
\hline Samples & $\begin{array}{l}\text { Reactive powder mortar } \\
\mathrm{w} / \mathrm{c}=0.4\end{array}$ & $\begin{array}{l}\text { White cement paste } \\
\mathrm{w} / \mathrm{c}=0.4\end{array}$ & $\begin{array}{l}\text { Ordinary Portland cement paste } \\
\mathrm{w} / \mathrm{c}=0.4\end{array}$ \\
\hline$\tau_{\mathrm{m}}$ & $0.7 \mathrm{~ns}$ & $1 \mathrm{~ns}$ & $0.8 \mathrm{~ns}$ \\
\hline $\mathrm{D}_{\text {surf }}=\varepsilon^{2} / 4 \tau_{\mathrm{m}}$ & $3.6 \times 10^{-7} \mathrm{~cm}^{2} / \mathrm{s}$ & $3.6 \times 10^{-7} \mathrm{~cm}^{2} / \mathrm{s}$ & $4.5 \times 10^{-7} \mathrm{~cm}^{2} / \mathrm{s}$ \\
\hline $\mathrm{D}_{\text {surf }} / \mathrm{D}_{\text {bulk }}$ & $1 / 60$ & $1 / 60$ & $1 / 50$ \\
\hline
\end{tabular}

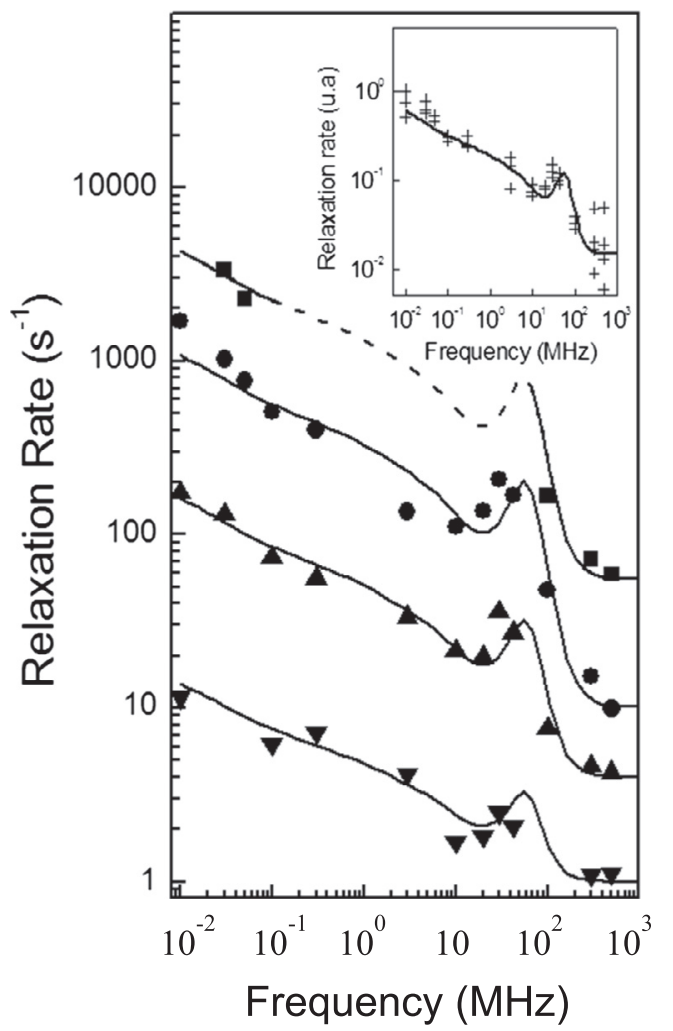

Fig. 11. Logarithmic plot of the four NMRD $1 / T_{1 i}=f_{i}\left(\omega_{0}\right)$ extracted from the inverse Laplace transform of the non-exponential longitudinal magnetization decay of a one-year-aged $C_{3} S$ synthetized sample [21]. The data have been obtained with different spectrometers whose frequencies range between $10 \mathrm{kHz}$ and $500 \mathrm{MHz}$. The normalization of all these four dispersion curves $1 / T_{1 i}$ to a single one, shown in inset, proves that the same relaxation processes (seen in Fig. 10b) occur for each of the four classes of $1 / T_{1 i}$ from 1 to $4000 \mathrm{~s}^{-1}$. According to the biphasic fast exchange model, these four NMRD curves are associated to the surface contribution of four non-connected pores; they have been fitted with Eq. (56) giving the four discrete average pore sizes $\left\langle R_{i}\right\rangle: 1.8,7.0,50$ and $600 \mathrm{~nm}$ (with different labels from the top to the bottom) for the studied $C_{3} S$ cement paste.

Here $\varepsilon=0.3 \mathrm{~nm}$ is the water molecule size. $x \varepsilon \sim 10 \AA$ with $x \in(1-3)$ according to previous calorimetry and NMR studies [66], and $S_{p, N M R}$ $=S_{p} F\left(\right.$ in $\left.\mathrm{m}^{2} / \mathrm{g}_{\text {liq }}\right)$ is the NMR-based value of the specific surface area, where $F$ is the ratio of the solid and liquid-proton magnetization densities at equilibrium $F=m_{\text {sol }}^{e q} / m_{w}^{e q} \ll 1[84,85]$. The distance of minimal approach between $I$ and $S$ spins is $r_{I S}=2.7 \AA$, $S=5 / 2$ for $\mathrm{Fe}^{3+}$ and $\mathrm{Mn}^{2+}$, and $n \sim 1$ is the average number of water molecules bound to $\mathrm{Fe}^{3+}$ ions. The unique cross-over frequency $\omega_{c} \sim 22 \mathrm{kHz}$ is indicative of a fundamental modification in the relaxation when the correlation time reaches the value $1 / \omega_{c}=7.2 \mu \mathrm{s}$. In Eq. (56), the correlation time $\tau_{c}$ of the nuclear paramagnetic relaxation is given by $1 / \tau_{c}=1 / \tau_{e x}+1 / T_{1 F e}$ where $\tau_{e x} \gg T_{1 F e}$ is the life-time of trapped water in the ligand field of the ferric ions. The electronic spinlattice relaxation time of the paramagnetic impurity $T_{1 F e}$ (of the order of $\left.10^{-11} \mathrm{~s}\right)$ is defined as $T_{1 F e}^{-1}\left(\omega_{S}\right)=H_{S}^{2} \tau_{v}\left[\left(1+\omega_{S}^{2} \tau_{v}^{2}\right)^{-1}+\right.$ $\left.4\left(1+4 \omega_{S}^{2} \tau_{v}^{2}\right)^{-1}\right][73,86]$ where $\tau_{v}$ is the correlation time for the electron-lattice relaxation interaction and $H_{S}^{2}$ is the intensity of the electronic spin fluctuations. Eq. (56) and the theoretical calculations displayed in Fig. 10b show that the effect of the nuclear paramagnetic relaxation is limited to a narrow band in the highest frequency range of the measurements. This contribution appears when a certain population of bound water molecules (typical values of $n \sim 1-2$ ) exists in the ligand field of $\mathrm{Fe}^{3+}$. The typical enhancement of the overall proton frequency dependence seen in the inset of Fig. 9a, after about ten hours of hydration, thus gives NMR evidence of such bound water. The best fit of the NMRD profiles of Fig. 9b with Eq. (56) gives a surface diffusion translational correlation time $\tau_{m}=0.7 \mathrm{~ns}$ that leads to a surface diffusion coefficient about two orders of magnitude less than the surface diffusion coefficient of water in bulk (Table 2). An almost universal value of $\tau_{m} \sim 1 \mathrm{~ns}$ has been found for several different cement-based materials, including reactive powder concrete (see Table 2) [87]. This clearly shows that nuclear spin relaxation is able to sense the local translational dynamics of proton species at the surface of CSH pores that are present in all these materials. These dynamics obtained by analysing NMRD measurements are in agreement with molecular dynamics simulations of proton species in the proximity of $\mathrm{CSH}$ interfaces [86].

Another representative model cement-based material is the tricalcium silicate $\mathrm{Ca}_{3} \mathrm{SiO}_{5}$ (the so-called $C_{3} S$ ) synthetized in the laboratory and which represents $60-70 \%$ of industrial Portland cement. Fig. 11 shows NMRD profiles $1 / T_{1}\left(\omega_{I}\right)$ obtained for a one-year-aged $C_{3} S$ synthetized sample with different spectrometers at frequencies between $10 \mathrm{kHz}$ and $500 \mathrm{MHz}$ [21]. The normalization of all these dispersion data to a single curve, as shown in the inset of Fig. 11, proves that the same relaxation processes (seen in Fig. 10b) occur for each of the four classes of $1 / T_{1 i}$ from 1 to 4000 $\mathrm{s}^{-1}$. The solid lines in Fig. 11 are best fits obtained using Eq. (56), including the nuclear paramagnetic relaxation contribution that is particularly visible as the "bump" at $60 \mathrm{MHz}$ on the inset of Fig. 11. The four NMRD curves reflect mainly the surface contribution of Eq. (56) that is weighted by the surface to volume ratio of the pores; these data give the following average discrete pore sizes $\left\langle R_{i}\right\rangle: 1.8,7.0,50$ and $600 \mathrm{~nm}$ for the cement paste.

Very controversial questions concern the measurement of specific surface area, $S_{p}$, of a hydrated cement-based material and how it behaves as a function of the duration of hydration. The measurement of $S_{p}$ is relevant to other chemically reactive nanoporous materials encountered in heterogeneous catalysis, membrane separation, chemical engineering, oil recovery and even in food science. Obtaining a directly measured and reliable value of $S_{p}$ is fundamental for characterizing the highly disordered internal porous microstructure present in these materials. Here the value of $S_{p}$ is directly related to the mechanical performance. However most of the existing techniques have been shown to be inadequate in many cases. For instance, widely dispersed values of $S_{p}$ ranging between 80 and $300 \mathrm{~m}^{2} / \mathrm{g}$ have previously been obtained [88], by methods including nitrogen gas sorption [89], mercury intrusion [90], small-angle X-ray (SAXS [91]) and neutron (SANS [92]) scattering and NMR relaxation [93-96]. The SAXS data fall systematically well above this range and differ from the SANS data performed on similar materials. The gas sorption method requires 


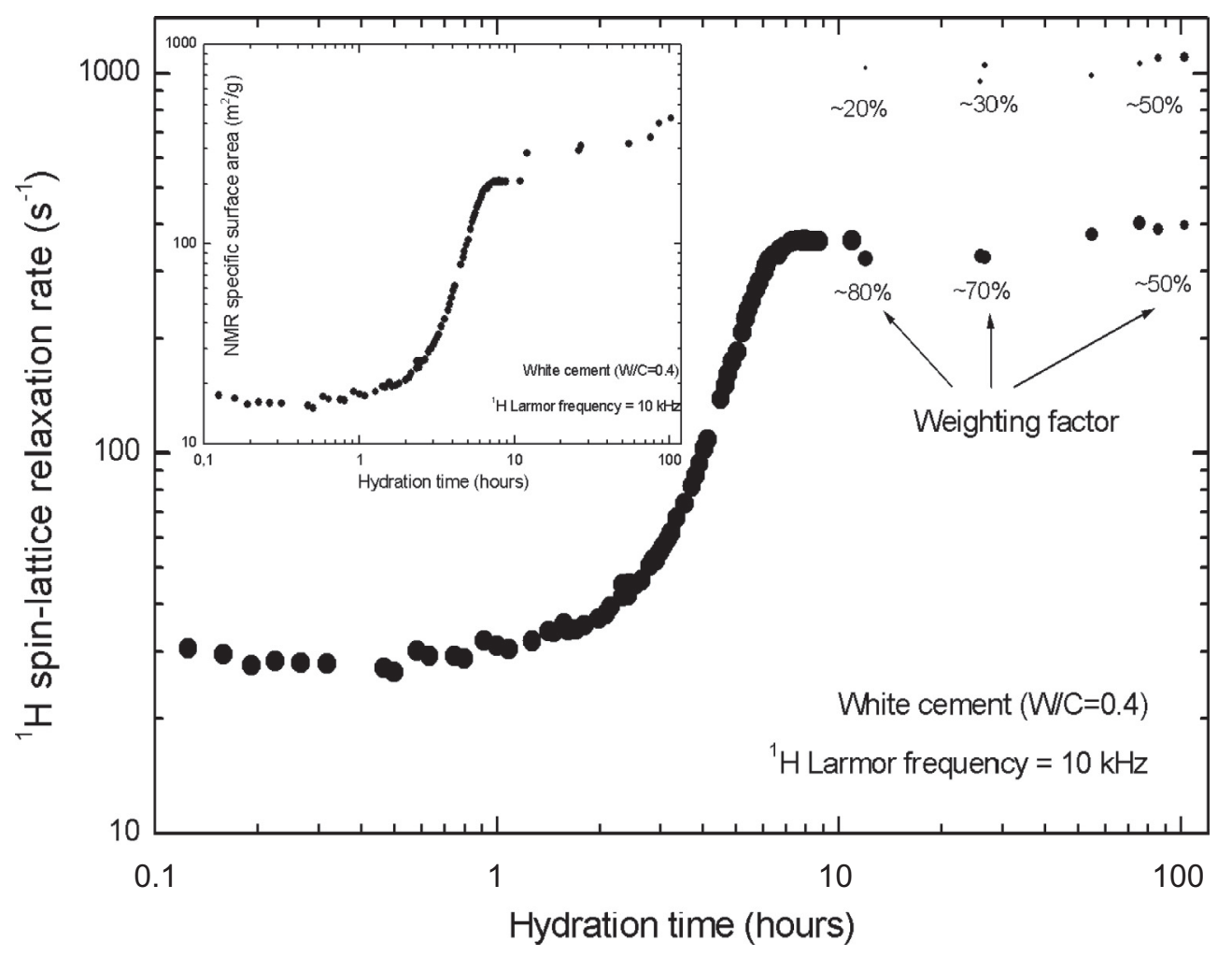

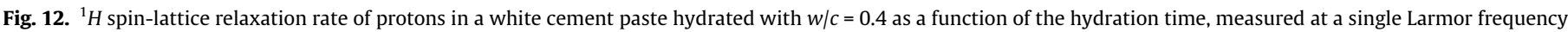

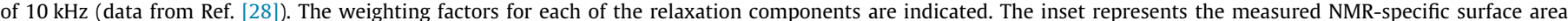
derived from the data in the main figure with use of equations given in Ref. [20].

a preliminary drying step and is limited in the pore sizes that can be measured. The mercury invasion method requires high pressures that perturb the porosity. NMR relaxation measurements for protons [93] and deuterons [94] were made only at a single high Larmor frequency, making it difficult to separate the surface and bulk contributions to the overall measured relaxation rate. Moreover, some of these NMR measurements require successive liquid saturation and drying or use of NMR cryoporometry [97] that perturb the microstructure. In contrast, NMRD allows direct probing of the surface area of a hydrated cement-based material without any requirement for drying or temperature modification [28]. The technique clearly separates the surface $\left(R_{1 \text { surf }}\right)$ and bulk $\left(R_{1 \text { bulk }}\right)$ contributions to the overall ${ }^{1} \mathrm{H}$ nuclear spin-lattice relaxation rate $\left(R_{1}\right)$ of water confined within the hydrated cement. In order to rule out any kinetic effects due to the continuous setting process, Korb et al. proposed a very fast measurement of the time evolution of the proton-water $1 / T_{1}(t)$ at a single frequency $(10 \mathrm{kHz})$ corresponding to the plateau on the NMRD profile in Fig. 9a [28]. The method is sensitive to both open and closed porosities over a wide range of length-scales, allowing the time evolution of proton species surface dynamics in different cement-based materials to be directly probed by measuring $1 / T_{1}(t)$ (inset of Fig. 12). The method is sufficiently fast ( $\sim 2 \mathrm{~min}$ ) that it can be applied continuously during the progressive hydration and setting of cement. At this low frequency, the overall relaxation is mainly due to the liquid proton species in interaction with the solid protons at the still growing CSH surfaces [20]. Fig. 12 shows the time evolution of $S_{p}$ for a white cement paste up to the first $100 \mathrm{~h}$ of hydration [28]. Similarly to results from the well-known isothermal conduction calorimetry measurement [98], Fig. 12 shows the three periods of the cement hydration, namely the dormant initial period, followed by setting due to the progress of chemical reaction after $4 \mathrm{~h}$ and lastly the transition to a period of slower diffusion at long times. It is well known in the cement industry that calorimetric measurements give a master curve from which can be obtained the degree of advancement of the chemical reaction at the start of the hydration process. The time evolution of the remaining water protons $1 / T_{1}(t)$ measured by NMRD at $10 \mathrm{kHz}$ as shown in Fig. 12 gives another master curve that reveals the decrease of water dynamics in the proximity of the growing solid surface of the CSH hydrates. It shows directly the time evolution of $S_{p}$ of the cement paste (inset of Fig. 12) that provides clear evidence of both the dormant period and the characteristic slow surface enhancement observed in the diffusive period.

\subsection{Direct probing of the nano-wettability of plaster pastes}

How does the microstructure of gypsum, a material of general interest in civil engineering, appears and evolves during hydration and setting, and how is it possible to follow these processes by non-destructive techniques? Essentially, there are two main objectives in such research on gypsum. (i) Improve the knowledge of the main steps of hydration of plaster to give key parameters for the mixing efficiency of this material. (ii) Improve the mechanical properties by a better knowledge of microstructure (specific surface area, pore size distribution and connectivity) of this much-used disordered solid system.

It is well known that proton NMR spectroscopy characterizes the proton chemical species within the gypsum crystal [99]. NMR proton relaxometry at low magnetic fields has been used during the setting of plaster, simultaneously varying the water to plaster ratio $(w / p)$ between 0.4 and 1 [18,19]. 2D spin correlation $T_{1}-T_{2}$ measurements have also been proposed to compare the hydration and microstructure in $\beta$-plaster with ground gypsum mineral and the effects of citric acid as an accelerant and retardant, respectively [100]. With NMR techniques, no drying is necessary that could 
produce nanostructure modifications by non-wetting fluid intrusion (as would be needed for nitrogen adsorption or mercury intrusion porosimetry). A comprehensive proton NMR relaxometry study was made [101] allowing the evolving microstructure of this
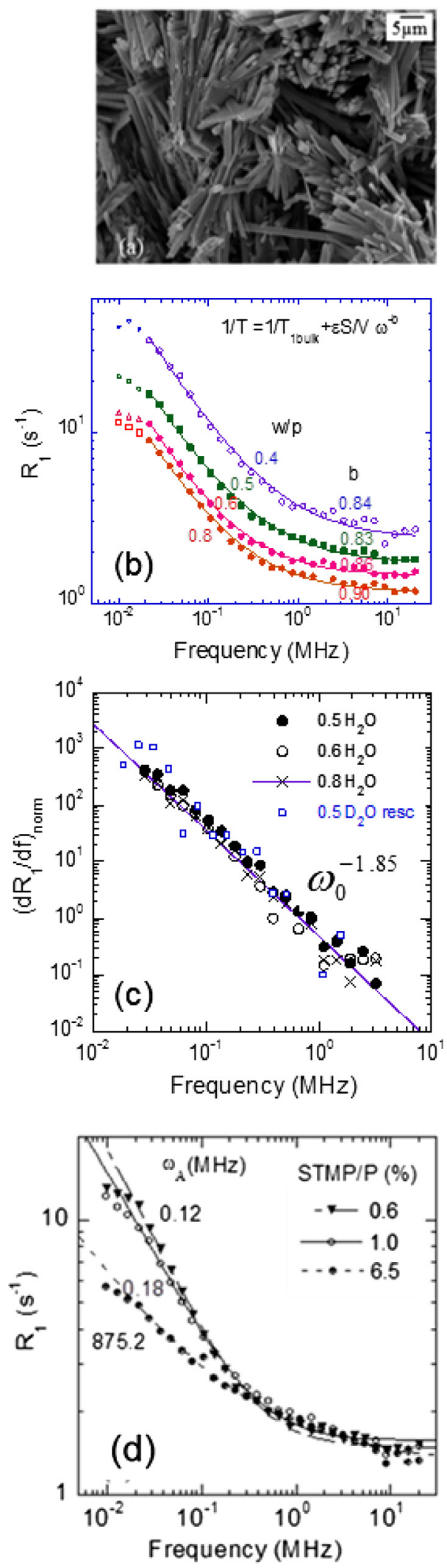

building material to be characterized directly and without perturbation. At least two transverse relaxation times were found corresponding to different water populations, showing two evolution modes of organization of the microstructure in the range of water to plaster ratios $(0.4 \leq w / p \leq 0.6)$ and $(0.7 \leq w / p \leq 1)$, in agreement with pulsed field gradient NMR experiments. A change of these populations was observed by adding an increasing amount of crushed gypsum, and the biphasic fast exchange model was verified by saturation and desaturation experiments [101]. Last, using an original model of transverse spin relaxation in presence of slow chemical exchange between the two detected populations gave an estimate of the rate $W$ of this process $[19,101]$. The variation of this rate $W$ with $w / p$ exhibits a clear gap between two different regimes, confirming the presence of the two evolution modes of organization. This gap occurs between $w / p=0.6$ and 0.7 and could be representative of a percolation threshold. This NMR method is sufficiently fast and robust to follow the progressive setting of the reactive interface of any porous medium without any dehydration.

Another question concerns the possibility of probing nondestructively the nano-wettability of a reactive porous plaster paste and how this nano-wettability changes with addition of additives. This question is very important in the field of civil engineering, as controlling the mechanical properties of such materials is of great interest in the building industry. This is all the more important for wetting water because it controls the kinetics of hydration and setting of the microstructure of such a widely used material. An in situ and non-invasive measurement of the NMRD features of the surface contribution of $1 / T_{1}$ arising from the different processes of molecular surface dynamics has been proposed for measuring the average lifetime of the wetting water directly on the pore surface of gypsum [102]. This technique has allowed a clear discrimination of the anisotropic dynamic motions on or in proximity to the local proton acceptors on the basal plane of gypsum needles as opposed to the fast isotropic molecular motions of water in bulk.

The hydration of calcium sulphate hemihydrate $\left(\mathrm{CaSO}_{4} \cdot 1 / 2 \mathrm{H}_{2} \mathrm{O}\right)$, usually called plaster, can be described the following way [103]. In a first step, the dissolution of the hemihydrate form leads to calcium and sulphate ions. Then, the crystallization of calcium sulphate dihydrate $\left(\mathrm{CaSO}_{4} \cdot 2 \mathrm{H}_{2} \mathrm{O}\right)$, usually called gypsum, starts as soon as the over-saturation is reached. Gypsum crystals are needle-shaped and lock in water molecules as shown by electron scanning electron microscopy (Fig. 13a). The evolution of plaster paste has been probed continuously, without perturbing the system, by using proton NMR transverse relaxometry of mixing water at a fixed magnetic field $[18,19]$. According to the well-known chemical reactions for hydration, the stoichiometric water to plaster weight ratio is 0.186 . The ratio generally used in the gypsum

Fig. 13. (a) Scanning electron micrograph of needle shaped of gypsum crystals that lock in water molecules. (b) ${ }^{1} \mathrm{H}$ NMRD profiles obtained for a plaster when increasing the water to plaster ratio $w / p$ from 0.4 to 0.8 . The continuous lines represent the best fits obtained with a linear combination of a constant (bulk value) and a power law of exponent $b \sim 0.85$. (c) Frequency dependencies of the derivative of the data displayed in (b) that removes the constant at large frequencies. The single continuous line represents now the best fit obtained with a power law $d R_{1} / d \omega_{0} \propto 1 / \omega_{0}^{1.85}$. This representation proves that all the data can be fitted with the same "apparent" power law $R_{1} \propto 1 / \omega_{0}^{0.85}$. The NMRD data obtained from ${ }^{2} \mathrm{H}$ of heavy water yield the same exponent of the power law, proving the intramolecular origin of the relaxation. (d) ${ }^{1} \mathrm{H}$ NMRD profiles obtained for a plaster with an increasing quantity (\%) of adsorbed sodium trimetaphosphate (STMP/P) adjuvant per gram of plaster $P$ [102]. The data fitted with Eqs. (58a), (58b) show the drastic increase of the frequency parameter $\omega_{A}$ introduced in Eq. (59) from $0.12 \mathrm{MHz}$ (without $S T M P / P$ ) to $875.2 \mathrm{MHz}$ when $S T M P / P=6.5 \%$. In this later case the data can be fitted with Eq. (60). 
industry lies between 0.4 and 1, i.e. much higher than 0.186 . Such an excess of water enables the development of porosity; the hardening of a plaster paste leads to a permeable porous structure, made of entangled needle-shape gypsum crystals (Fig. 13a). To investigate this system by NMRD, Korb and Levitz [102] prepared different samples by manual mixing of hemihydrates powder and distilled water, at well-defined water to plaster weight ratios $(w / p)$ from 0.4 to 0.8 , in some cases also including sodium trimetaphosphate adjuvant (STMP) to change the hydration kinetics and microstructure. NMRD profiles of proton and deuterium longitudinal relaxation rates $R_{1}$ were measured at various stabilized temperatures, always after the setting period $(60 \mathrm{~min})$ was complete so that hydration is complete and microstructure does not evolve. Provided non-magnetic tools were used for preparing the plaster paste, ESR showed paramagnetic impurities to be absent. Fig. 13b shows NMRD profiles for water-to-plaster weight ratios $(w / p)$ between 0.4 and 0.8 at $25^{\circ} \mathrm{C}$; below a cross-over frequency of $22 \mathrm{kHz}, 1 / T_{1}$ tends progressively to a constant value, while at higher frequencies it seems to follow an apparent power law plus a constant, $R_{1}=1 / T_{1} \sim A \omega^{-0.85}+C$, over more than three orders of magnitude (Fig. 13b) [102]. The exponent of this power law is preserved for water-to-plaster weight ratios between 0.4 and 0.8 and temperatures between 25 and $45^{\circ} \mathrm{C}$ (Fig. 13b). The first derivation of the NMRD data of Fig. 13b suppresses the constant values at high frequencies and presents power laws with the same exponents $(-1.85)$ for all the water-to-plaster weight ratios measured $(w / p)$ (Fig. 13c). Importantly, one observes the same
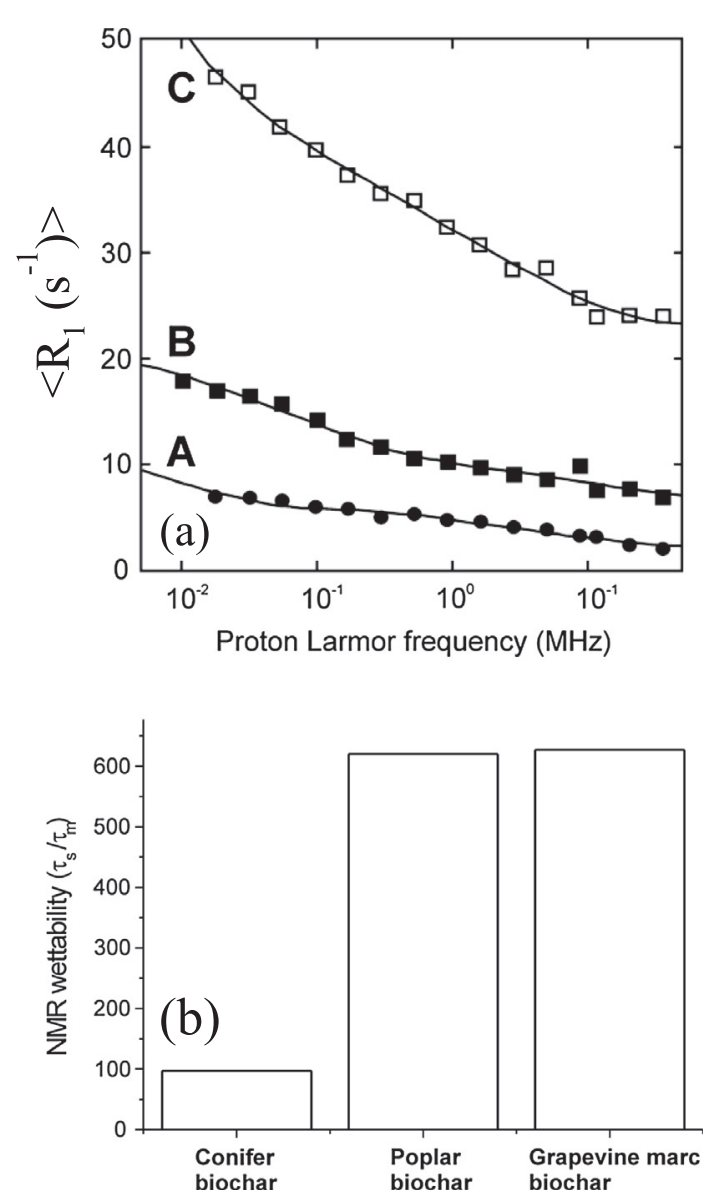

Fig. 14. (a) NMRD profiles of the logarithmic average of the fastest relaxing water distributions $\left\langle R_{1}\left(\mathrm{~s}^{-1}\right)\right\rangle$ in contact with biochar surfaces obtained from conifer $(\mathrm{A})$, poplar (B) and grapevine marc (C). (b) Surface dynamical activity or NMR-derived wettability obtained by fitting the NMRD dispersion data in (a) using Eq. (55). The data come from Ref. [34]. power-law behaviour for either NMRD profiles of $\mathrm{H}_{2} \mathrm{O}$ or of $\mathrm{D}_{2} \mathrm{O}$ (Fig. 13c) [102]. This is a strong argument in favour of an intramolecular diffusive relaxation process on and in proximity of the entangled needle-shape gypsum flat surface. However, one observes an anomalous behaviour of $R_{1}$ that increases with the temperature, thus revealing an interaction with the solid surface. Last, one observes in Fig. 13d a net decrease of the exponent of the apparent power law up to a true power law $1 / T_{1} \sim \omega^{-0.50}$ in presence of increasing quantities of adsorbed sodium trimetaphosphate (STMP) adjuvant. To interpret this, the authors proposed an analytical model of the NMR relaxometry based on the biphasic fast exchange model [102] in which the overall $R_{1}$ is defined to be a superposition of bulk and surface contributions:

$R_{1}\left(\omega_{0}\right)=R_{1, \text { Bulk }}+\frac{\lambda S}{V} R_{1, \text { Surface }}\left(\omega_{0}\right)$

The bulk contribution $R_{1, \text { Bulk }}$ is constant in the frequency range studied [43]. The frequency dependence of the surface contribution $R_{1, \text { Surface }}$ was calculated with the Levitz model [104,105], which involves elementary intermittent time steps near the interface (e.g. bulk bridges, adsorption trails and escaping trails):

$R_{1, \text { Surface }}\left(\omega_{0}\right) \propto\left[J_{\text {Surface }}\left(\omega_{0}\right)+4 J_{\text {Surface }}\left(2 \omega_{0}\right)\right]$,

$J_{\text {Surface }}\left(\omega_{0}\right) \propto \tau_{A}\left[\sqrt{\frac{\omega_{0}}{\omega_{A}}}+\frac{\omega_{0}}{\omega_{A}}+\frac{1}{2}\left(\frac{\omega_{0}}{\omega_{A}}\right)^{3 / 2}\right]^{-1}$,

where

$\omega_{A}=\delta^{2} /\left(2 D \tau_{A}^{2}\right)$

is a characteristic frequency $(\times 2 \pi)$ related to the water size $\delta=0.3 \mathrm{~nm}$, the translational bulk diffusion coefficient $D$ of water and the average adsorption correlation time $\tau_{A}$. Fig. 13d shows that this

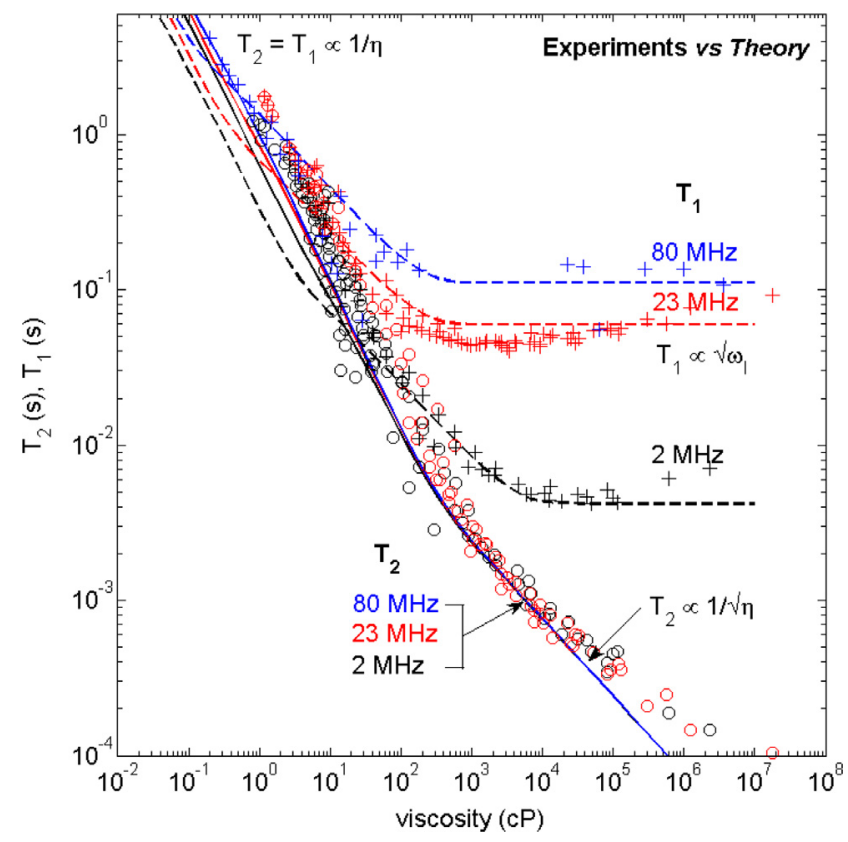

Fig. 15. Variations of $T_{1}$ and $T_{2}$ over a large range of viscosity $\eta(c P)$ for a large set of different alkanes and crude oils at room temperature; experimental data (points) and theoretical predictions (lines) are shown for logarithmic average relaxation times ( $\left\langle T_{1,1 M}\right\rangle$ and $\left\langle T_{2,1 M}\right\rangle$, labeled $T_{1}$ and $T_{2}$ ) and were obtained at 2 (black), 23 (red), and $80 \mathrm{MHz}$ (blue). The experimental points ( $T_{1}$ crosses; $T_{2}$ circles) are taken from Refs. $[110,113,114,127]$. The theoretical values (continuous and dashed lines) were obtained using Eqs. (61a), (62), (64)-(66) with the following parameters: $R_{\text {agg }}=2.6 \mathrm{~nm}, R_{\text {mol }}=0.39 \mathrm{~nm}, \delta_{I S}=0.855 \mathrm{~nm}$ for $T_{2}$ and $0.655 \mathrm{~nm}$ for $T_{1}, \eta_{C}=250 \mathrm{cP}$ for $T_{2}$ and $160 \mathrm{cP}$ for $T_{1}$ (except at $T_{1}$ at $2 \mathrm{MHz}$ where $\eta_{C}=3500 \mathrm{cP}$ ). 


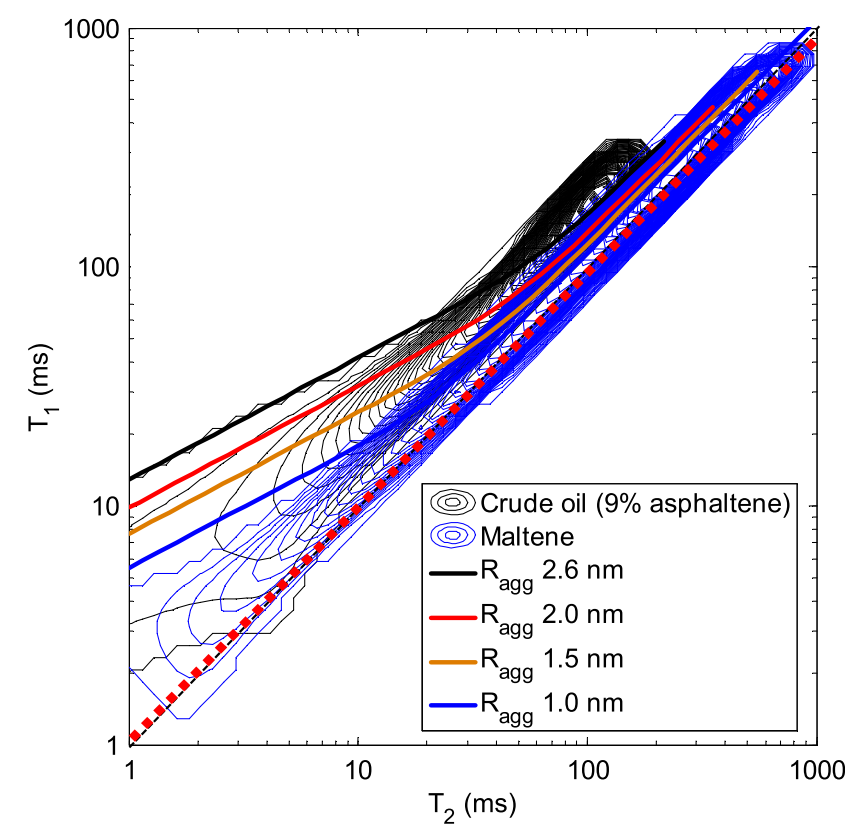

Fig. 16. Experimental and theoretical 2D $T_{1}-T_{2}$ spin-correlation plots at $2.5 \mathrm{MHz}$. Experimental data taken from Fig. 7 of Ref. [110]. Theoretical values (continuous lines) were obtained using Eqs. (62), (64) and (65) for the indicated different radii of the asphaltene macro-aggregates $R_{\text {agg. }}$. Both experiments and theory display an upward deviation away from the $T_{1}=T_{2}$ red dashed line for short $T_{1}$ and $T_{2}$ values.

single-parameter model is supported by the experimental data, and that this characteristic frequency does not vary much $\left(\omega_{A} \sim 0.1-0.2\right.$ $\mathrm{MHz}$ ) with $w / p$ (Fig. 13d). According to Eqs. (58a), (58b), this gives an adsorption time scale about $\tau_{A}=5.0 \mathrm{~ns}$. These relaxation features prove that, as expected without STMP, the surface relaxation process does not change with $w / p$ or temperature because the surface conditions do not change much. In contrast, Fig. 13d shows that this is not the case in the presence of increasing quantity of STMP adjuvant in the wetting water. Here, $R_{1}$ decreases and $\omega_{A}$ drastically increases up to $875.2 \mathrm{MHz}$ in presence of $6.5 \%$ of STMP adjuvant (Fig. 13d), and correspondingly the adsorption time scale decreases to $\tau_{A}=64$ ps with $6.5 \%$ of adjuvant. These drastic changes might be explained by the presence of STMP adjuvant at the surface of the largest 010 planar structure of gypsum, which would result in a net diminution of accessible bonding sites for water. The frequency dependence given by Eqs. (58a), (58b) thus simplifies and tends now to the following linear combination of single power laws:

$R_{1}\left(\omega_{0}\right) \propto R_{1, B u l k}+A \omega_{d}^{2} \tau_{A}\left[\left(\frac{\omega_{0}}{\omega_{A}}\right)^{-1 / 2}+4\left(\frac{2 \omega_{0}}{\omega_{A}}\right)^{-1 / 2}\right]$,

when $\omega_{0} \ll \omega_{\mathrm{A}}$

where $A$ is a constant and $\omega_{d}$ is the intramolecular dipole-dipole frequency for the protons of water. This expression allows easy determination of the average adsorption time of residence for water $\tau_{A}$ on the gypsum polycrystals from the NMRD data. The effect seen for $R_{1}\left(\omega_{0}\right)$ shows that the average time scale $\tau_{A}$ depends directly on the physical chemistry at the surface, and is indicative of the dynamical wettability of the material at the nanoscale. As the degree of hydration during setting is directly related to the mechanical properties such as compressive strength and modulus of elasticity [19], these results indicate that the parameter $\tau_{A}$ controls most physicochemical properties of the porous structure of this material.

\subsection{Surface affinity of water in biochars}

Many soil functions depend on the interaction of water with soil. The affinity of water for soils can be altered by applying soil
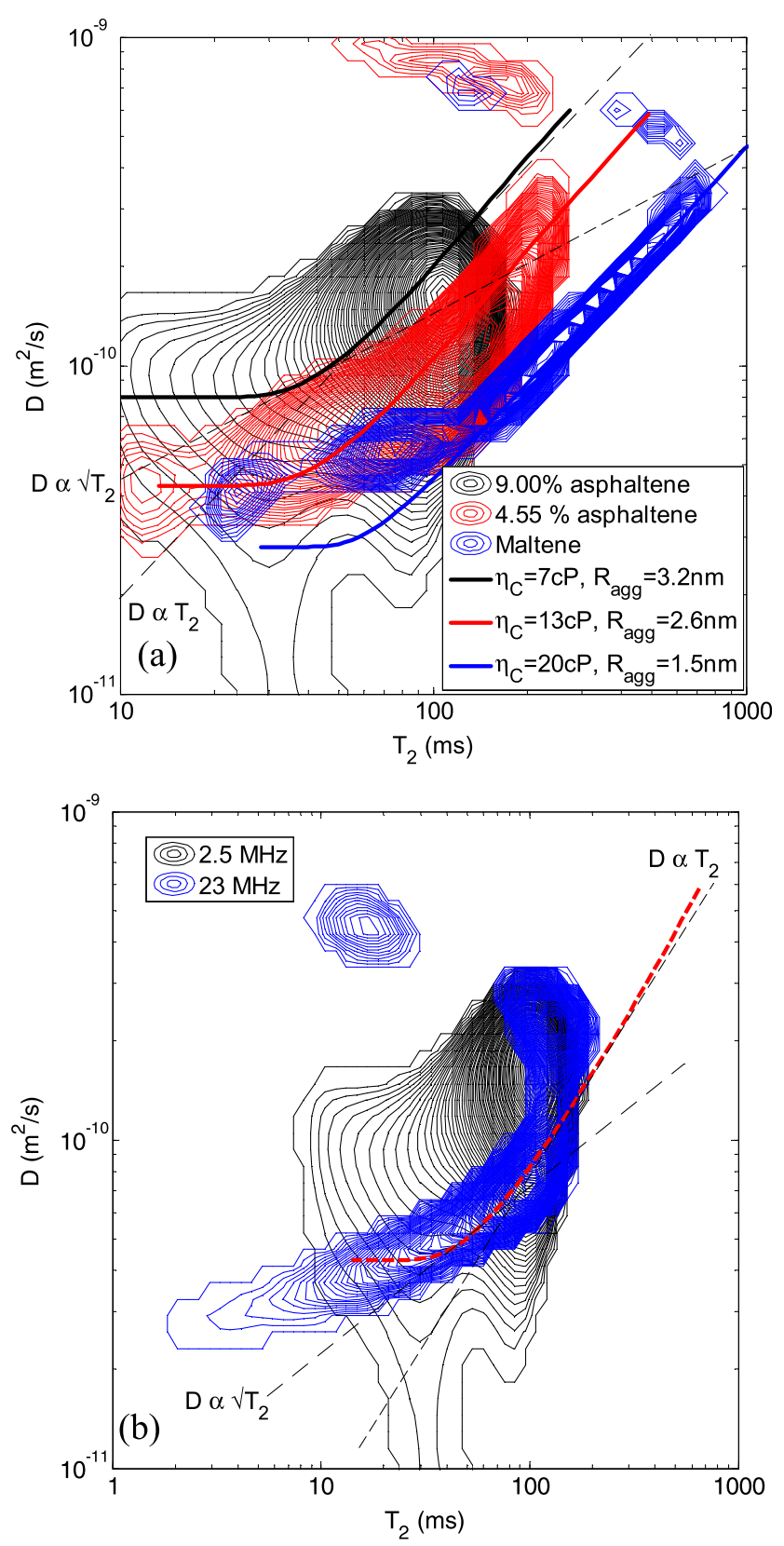

Fig. 17. (a) Experimental data (from Ref. [110]) and theoretical $D-T_{2}$ correlation plots obtained at $2.5 \mathrm{MHz}$ for a native crude oil (9 wt\% asphaltene; black), $4.55 \mathrm{wt} \%$ asphaltene (red), and maltene (blue). Theoretical values were obtained using Eqs. (62), (64) and (65) with the following parameters: $R_{m o l}=0.39 \mathrm{~nm}, \delta_{I S}=0.855 \mathrm{~nm}$. One clearly observes the upward deviation of the diffusion coefficient $D$ for short $T_{2}$ values. (b) Experimental $D-T_{2}$ correlation maps performed at 2.5 (black) and 23 $\mathrm{MHz}$ (blue) for a native crude oil containing $9 \%$ (wt) asphaltene. The red dashed line is the theoretical $D-T_{2}$ plot calculated from Eqs. (64) and (65) with the same parameters as in Fig. 16. The data clearly show the upward deviation of the diffusion coefficient $D$ for short $T_{2}$. Experimental values taken from Fig. 4 of Ref. [110].

amendments such as stone meal, manure or biochar (a carbonaceous material obtained by pyrolysis of biomasses). In fact, the addition of hydrophobic biochar to soil may increase soilrepellency, reduce water-adsorbing-capacity, inhibit microbialactivity, and alter soil filter-, buffer-, storage-, and transformation functions. For this reason, it is important to monitor the water affinity of biochar surfaces (i.e. their wettability) when addressing their applications in soil systems. Bubici et al. found that either NMR or contact angle (CA) results revealed the same wettability 
trend for the biochars they studied [34]. However, the advantage of NMR relaxometry over CA measurements lies in the possibility of obtaining a variety of different information at the microscopic level in a single experiment. In fact, while CA provides only macroscopic wettability, NMR relaxometry allows a description of the mechanisms for water molecular dynamics on biochar surfaces, thereby
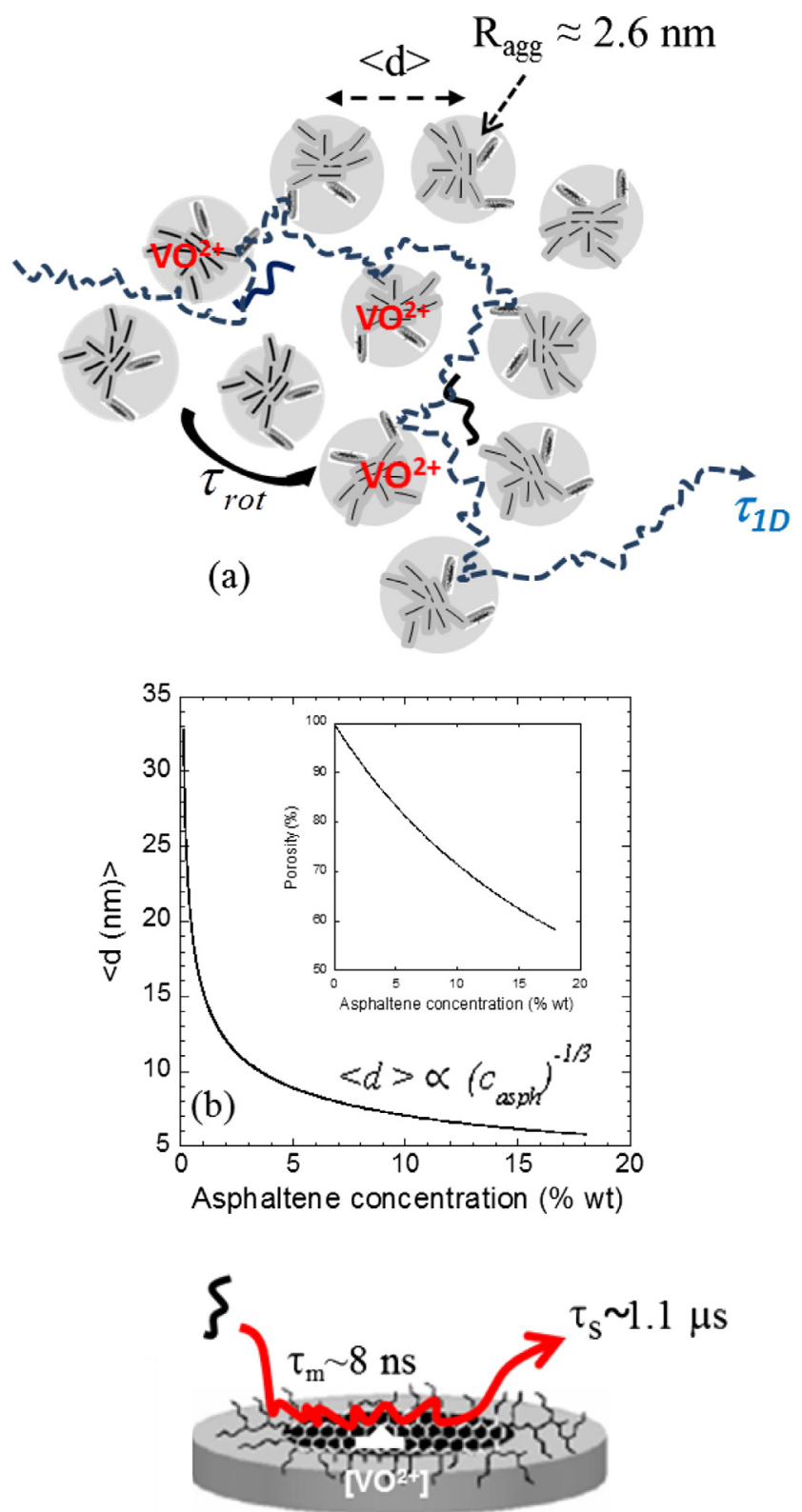

(c)

Fig. 18. (a) Schematic diagram representing the transient porous network of asphaltene macro-aggregates characterized by a radius $R_{a g g}$ and a rotational correlation time $\tau_{\text {rot }}$. The paramagnetic $\mathrm{VO}^{2+}$ ions are indicated schematically within the macro-aggregates. The tortuous dashed line represents the quasi-1D translational diffusion of hydrocarbons characterized by a molecular size $R_{m o l}$ and a correlation time $\tau_{1 D}$. (b) Calculated variation of the average distance of separation $\langle d\rangle$ between these macro-aggregates as a function of asphaltene concentration $c_{a s p h}$ (wt\%). The inset shows the calculated variation of the effective porosity with $c_{a s p h}$ (wt\%). (c) Schematic diagram representing the translational diffusion of hydrocarbons (C8-C12) characterized by the surface translational correlation time $\tau_{m}$ and the time of residence $\tau_{s}$ in proximity to the vanadyl ions $\mathrm{VO}^{2+}$ (labeled as a white pyramid) at surfaces of slowly rotating asphaltene nano-aggregates. This localization of vanadyl paramagnetic ions comes from the molecular dynamics simulations of Ref. [121]). leading to the possibility of understanding the role of biochar in increasing soil quality and plant nutrition. In fact a bimodal distribution of water molecules experiencing different environments was identified in each biochar/water mixture [34]. The narrow distribution of water molecules centered about $T_{1} \sim 2.5 \mathrm{~s}$ is due to the oxygen dissolved in bulk water. Conversely, the distribution of molecules relaxing at shortest $T_{1}$ values was associated with water directly in contact (either mobile or immobile) with the biochar surface. In the discussion below, only this second type of water molecules were accounted for. Fig. 14a shows the NMRD profiles of the logarithmic average $\left\langle R_{1}\left(\mathrm{~s}^{-1}\right)\right\rangle$ of the fastest relaxing water distributions in contact with biochar surfaces obtained from conifer (A), poplar (B) and grapevine marc (C) [34]. As earlier reported in Pasquale et al. [106], the $R_{1}$ values vary in the order: $R_{1 \text { (conifer biochar) }}<R_{1 \text { (poplar biochar) }}<R_{1 \text { (grapevine marc biochar) }}$ over the whole range of magnetic fields studied. Fig. $14 \mathrm{~b}$ shows the $\tau_{s} / \tau_{m}$ ratios obtained by Bubici et al. for the three biochars used, obtained using a spin-relaxation theory similar to that leading to Eq. (55), which resulted in the order $\left(\tau_{s} / \tau_{m}\right)_{\text {conifer biochar }}<$ $\left(\tau_{s} / \tau_{m}\right)_{\text {poplar biochar }} \approx\left(\tau_{s} / \tau_{m}\right)_{\text {grapevine marc biochar }}$. These results suggest that the microscopic NMR-derived affinity data can be related to the macroscopically observable wettability as measured by contact angle CA. Moreover, the agreement between the NMR relaxometry and contact angle measurements suggests a possible conservation of wettability mechanisms between nano- and macro-scale dimensions [34].

\section{Applications to petroleum fluids}

Crude oils are raw natural petroleum resources essentially composed by complex mixtures of many different hydrocarbons fractionated into saturated, aromatic, resins and asphaltenes (characterized by the SARA analysis [107]). Asphaltene molecules are defined as a fraction of petroleum defined by their solubility class. They are insoluble in light n-alkane solvents like n-pentane or nheptane and soluble in aromatic solvents like benzene or toluene [108]. The structure of asphaltenes is rather complex and usually

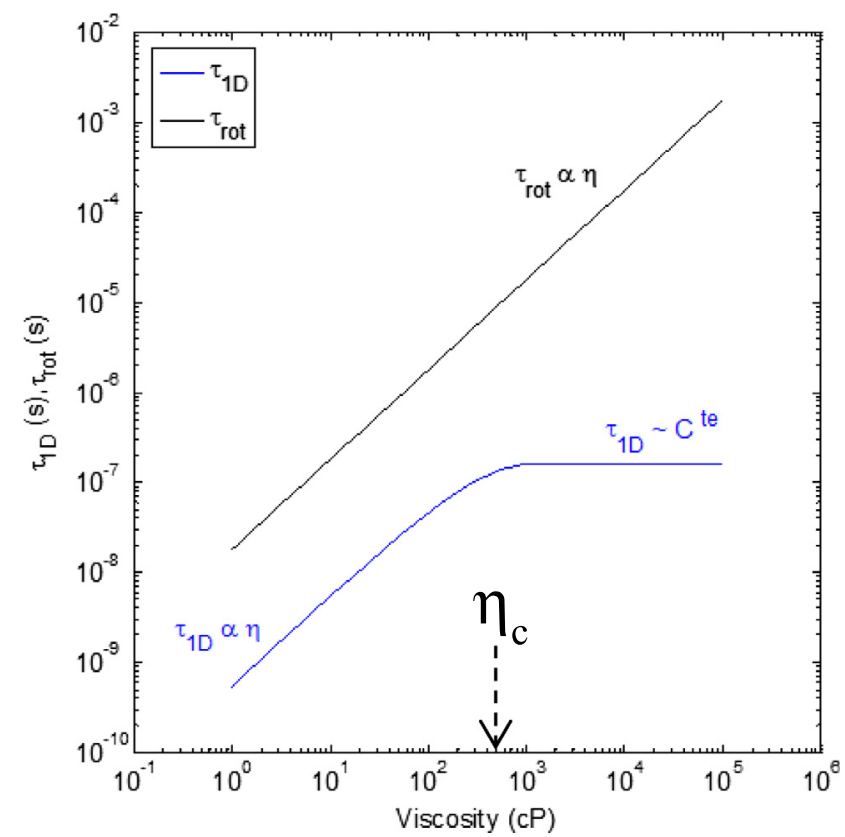

Fig. 19. Variations of the correlation times $\tau_{\text {rot }}$ and $\tau_{1 D}$ as a function of viscosity $(c P)$, calculated using Eqs. (61a) and (62). The figure indicates the threshold viscosity $\eta_{c} \sim 300 \mathrm{cP}$ between the usual behaviours $\tau_{1 D} \ll \tau_{\text {rot }} \propto \eta$ and the oilwetting relation $\tau_{1 D} \sim$ Cte obtained from Eq. (62) for large viscosities. 
assumed to consist of polycyclic aromatic hydrocarbons substituted with alkyl side chains and the presence of heteroatoms, including nitrogen, sulphur, oxygen, and trace metals ( $\mathrm{Ni}$ and $\mathrm{V}$ ) [109]. Asphaltene molecules represent a form of oil poisoning that could be responsible for pore blocking in oil reservoirs as well as in catalysts. They cause many problems in crude oil transportation, refinery and production, it is thus crucial to have a clear understanding of their dynamical behaviour in crude oils for improving crude oil production. The asphaltene concentration in crude oils, all over the world resources, varies from a few per cent up to $15 \%$ wt. Instead of studying a large number of crude oils of different asphaltene concentrations, it is easier to use only a single native crude oil of high asphaltene concentration and to derive from this sample some others with lower asphaltene concentrations by dilution with its own maltene using a known protocol of the petroleum industry (see Section 2.1 of Ref. [110]). The other components in crude oils such as "saturated", "aromatic" and "resin" components of the SARA analysis [107] are soluble in nalkane solvents and defined as maltenes. There are also different paramagnetic species in crude oils that have a great importance in the NMR measurements such as vanadyl ions $\mathrm{VO}^{2+}$ and organic carbon radicals that can be clearly identified by ESR spectra $[111,112]$. Our main objective here is to review how NMRD and 2D $T_{1}-T_{2}$ or $D-T_{2}$ techniques can be useful for characterizing, on different length- and time-scales, the dynamical properties of hydrocarbons in presence or absence of asphaltenes.

\subsection{Relations between NMR relaxation times, diffusion coefficients and viscosity of heavy crude oils}

Knowing the viscosity of a crude oil as early as possible during exploration is vital to the oil industry because it impacts the productivity, and the choice of recovery strategies. NMR relaxation is used for estimating the viscosity of crude oils, even down-hole, through measurements of longitudinal and transverse nuclear spin-lattice relaxation times provided that the BPP relationships (Eqs. 4a, 4b) between these relaxation times and the viscosity are firmly established [44].

For bulk light oils (low viscosity) the molecular motions of all saturated and aromatic hydrocarbon components are fast enough to average the magnetic dipolar fields of neighbouring spin systems (extreme narrowing limit), which results in the usual relation $T_{1} \propto 1 / \eta$ consistent with the standard calculation of relaxation times based on the Stokes-Einstein relation between the translational diffusion coefficient and viscosity (see Ref. [43]). However, for bulk heavy crude oils (high viscosity), the molecular motions of asphaltenes (see below), resins, and other high molecular weight structures are not fast enough to completely average the local dipolar fields of neighbouring spin systems, which results in large distributions of $T_{2}$ and $T_{1}$ that reflect the large diversity of molecular sizes. The situation is interesting because the observed viscosity dependencies of the "log-mean averages" (defined as: $\left\langle\frac{1}{T_{1,2, L M}}\right\rangle=\prod_{i=1}^{n}\left(\frac{1}{T_{1,2, i}}\right)^{f_{i}}=10^{-\sum_{i=1}^{n} f_{i} \log T_{1,2, i} / \sum_{i=1}^{n} f_{i}}$ where $f_{i}$ is the $i$ th value of the discretized relaxation time distributions $f\left(T_{1}\right)$ or $f\left(T_{2}\right)$ ) observed for a crude oil, follow the universal master curve displayed in Fig. 15 [113] that is very different from that given by the well-known BPP relaxation theory [44] that underlies Eqs. (4a, 4b). In particular, the anomalous nuclear spin relaxation features found for heavy crude oil include the following relaxation features: (i) $T_{2}$ is shorter than $T_{1}$; (ii) $T_{2} \propto 1 / \sqrt{\eta}$ and is almost independent of the Larmor frequency $\omega_{0} / 2 \pi$; (iii) above a certain viscosity, $T_{1}$ is almost independent of viscosity but depends strongly on the Larmor frequency $T_{1} \propto \sqrt{\omega_{0}}$. (iv) two-dimensional spincorrelation plots $T_{1}-T_{2}$ and $D-T_{2}$ have been also used for measuring the molecular dynamics over different length- and time-scales [110]. For heavy crude oils, the presence of asphaltenes creates some anomalous features in the observed two-dimensional correlation plots displayed in Figs. 16 and 17, respectively [114] that will be discussed in Sections 8.1.3 and 8.1.4. Asphaltenes have been shown to associate at higher concentrations, making complex particles that in turn may aggregate to make macro-aggregates $[109,115]$. There are still long-standing debates in the literature about their molecular architecture. Several authors are in favour of what are described as "archipelago aggregates" [116], while some others favour a so-called "inland continental" [109]. Recently, a breakthrough was made by using atomic force microscopy and molecular imaging of these aggregates [117]. Recent molecular dynamics simulations on realistic "inland continental" structure of asphaltenes show the role of hetero-atoms such as nitrogen and sulphur in the aggregation processes [118,119]. Thus, oils with large asphaltene concentrations may have considerable local structure, resulting in different effects on macroscopic properties such as viscosity and on micro-dynamic properties such as molecular reorientations and translations. Singer et al. have recently proposed another interpretation of the viscosityfrequency dependences of $T_{1}$ and $T_{2}$ for polymer-heptane mixes based on intramolecular ${ }^{1} \mathrm{H}-{ }^{1} \mathrm{H}$ dipole-dipole interactions and distributions of correlation times [120]. However, their model cannot account for the net frequency dependence $\left(T_{1} \propto \sqrt{\omega_{0}}\right)$ as well as the frequency independence of $T_{2}$ observed in Fig. 15 for various crude oils.

\subsubsection{Dynamic model for hydrocarbons diffusing in bulk crude oils in the presence of asphaltene}

Semi-classical treatments are always needed for relating the measured macroscopic relaxation times $T_{1}$ and $T_{2}$ to the various microscopic molecular correlation times seen in Fig. 18. For a crude oil of viscosity $\eta$, the correlation time $\tau_{\text {rot }}$ for the rotational diffusion of asphaltene macro-aggregates of radius $R_{\text {agg }}$ and the translational correlation time $\tau_{1 D}$ of hydrocarbon molecules of radius $R_{m o l}$ can be estimated by the Stokes-Einstein relations, which assume an isotropic environment and approximately spherical molecules (Fig. 18a):

$$
\begin{gathered}
\tau_{\text {rot }}=\frac{4 \pi R_{\text {agg }}^{3} \eta}{3 k_{B} T}, \\
\tau_{1 D}=\frac{12 \pi R_{\text {mol }}^{3} \eta}{k_{B} T} .
\end{gathered}
$$

For a native crude oil of viscosity $\eta=40 \mathrm{cP}$, Eq. (61a) yields $\tau_{\text {rot }} \sim 0.72 \mu$ s with $R_{\text {agg }}=2.6 \mathrm{~nm}$ and $\tau_{1 D} \sim 21.3 \mathrm{~ns}$ for $R_{\text {mol }}=0.39$ $\mathrm{nm}$ corresponding to $\mathrm{C} 8-\mathrm{C} 12$ hydrocarbon chain lengths. The fact that $\tau_{1 D} \ll \tau_{\text {rot }}$ justifies the assumption of a very slow rotation of the asphaltene macro-aggregates in comparison to the relatively fast translational dynamics of the hydrocarbons. For an asphaltene concentration of $c_{a s p h}=9 \mathrm{wt} \%$, the distance between macroaggregates is approximately $\langle d\rangle=7.3 \mathrm{~nm}$, which gives a very small pore space about $2 \mathrm{~nm}$, ensuring a quasi 1D translational diffusion for most of hydrocarbon-distribution within the low porosity of the transient porous network of quasi immobile asphaltene macro-aggregates (Fig. 18b). In the following, we aim at modelling the translational diffusion of hydrocarbons in proximity to vanadyl ions at the surface (or in proximity) of asphaltene nanoaggregates (Fig. 18c). Recent molecular dynamics simulations [121] show that the vanadyl ions $\mathrm{VO}^{2+}$ are included in metalloporphyrin molecules and are located only at surfaces of asphaltene nano-aggregates because of the pyramidal geometry of such metallic paramagnetic ions (Fig. 18c) that stands perpendicular to the four-nitrogen plane [121]. The network of asphaltene nano-aggregates and macroaggregates (Fig. 18a) appears to be similar to a transient 
(a) Experiments for oil-wet

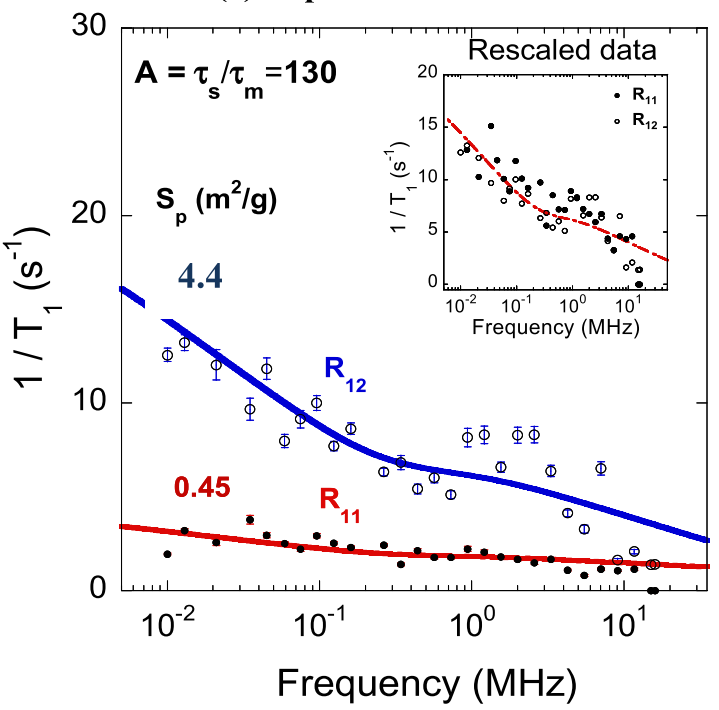

(c) Diphasic saturation

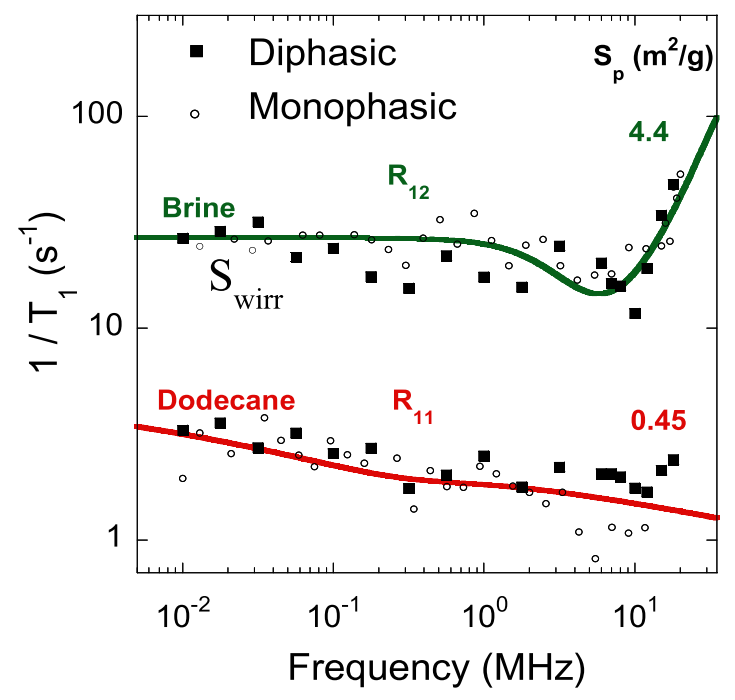

(b) Experiments on $\mathrm{S}_{\mathrm{w}}=100 \%$

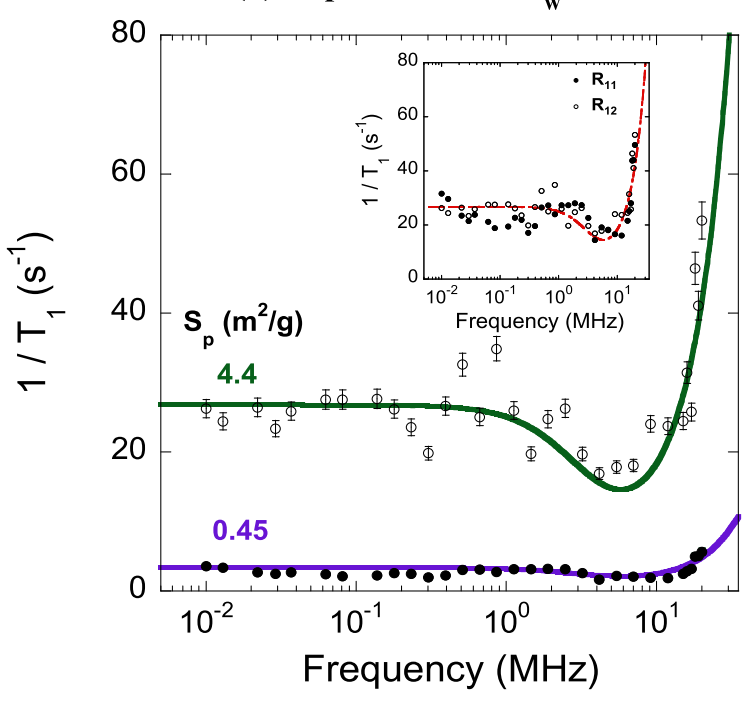

(d) Dual porosity extracted from (c)

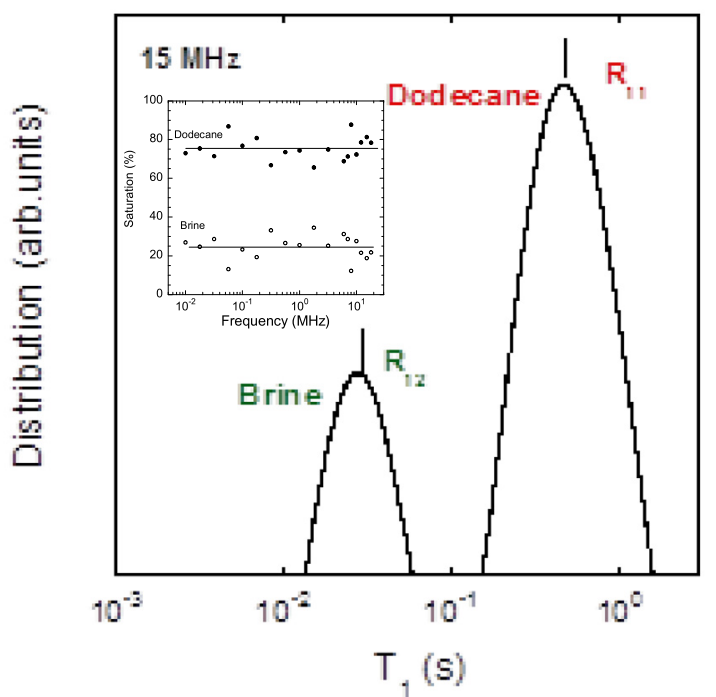

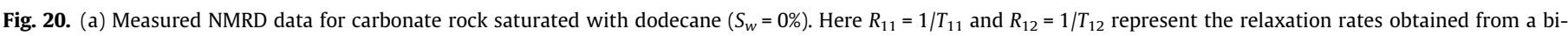

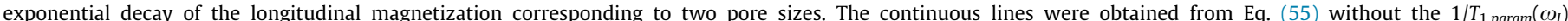

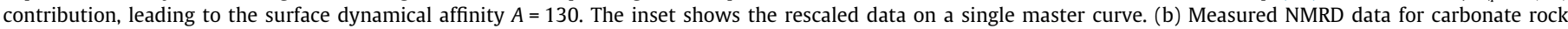

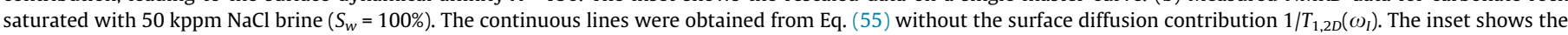

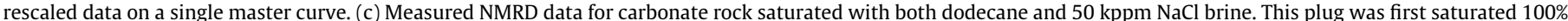

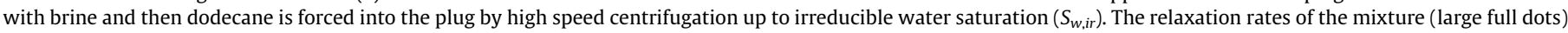

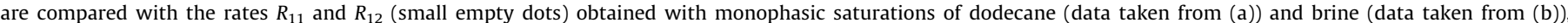

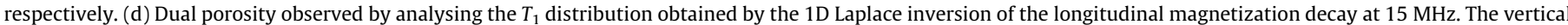

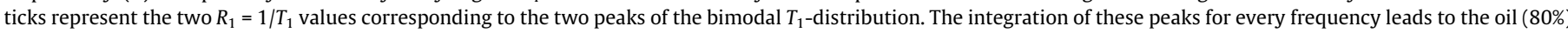

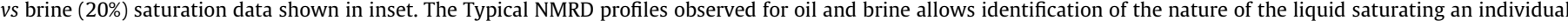
pore. All the data come from Ref. [27].

percolation network in disordered porous media. A similar "single-file" diffusion situation has been considered for solventadded processes associated with heavy oil production [122]. In this form of confinement, the Stokes-Einstein relation (Eq. 61a), can still be fulfilled for a quasi-spherical macro-aggregate. However, Eq. $61 \mathrm{~b}$ becomes questionable for defining $\tau_{1 D}$ when the saturated hydrocarbons have molecular sizes comparable to the average pore sizes of the transient porous network $\langle d\rangle$ (Fig. 18a and b). Therefore the hydrocarbon dynamics become strongly dependent on the confinement as well as the interaction between hydrocarbons and asphaltene. Why is there a maximal size of asphaltene macro- aggregates in Fig. 18a and what is the functional form for $\tau_{1 D}$ in such confinement? A plausible explanation is given in terms of an analogy between the equilibrium structure achieved with the approximately binary fluid of quasi-immobile asphaltene macroaggregates and hydrocarbons with the case of phase separation by which the two components of a diffusive binary fluid spontaneously separate and form pure domains of each component (as in spinodal decomposition). It is known that the non-linear Cahn-Hilliard equation [123] describes such a phase separation. At equilibrium and very far from the second-order phase transition where the two particles seek their own kind, the Cahn-Hilliard 
equation can be linearized and the segregation of such a binary mixture is characterized by thin transition layers between the segregated domains with a normal concentration profile given by the equilibrium function, $u(x)=\tanh (x / \sqrt{ }(2 a))$, where $x$ denotes a locally defined normal coordinate to the interface and $\sqrt{ } a$ corresponds to the length of the transition regions between the domains. As expected, this function tends to $u= \pm 1$ for sufficiently large $x$ compared to $\sqrt{ } a$ indicating separated domains. In the equilibrium situation of Fig. 18a, this analogy may be expressed as $\langle d\rangle \sim \sqrt{ }(a)$. One thus notes that the thickness of the layer between the macro-aggregates and the hydrocarbon phases becomes exponentially thin when the asphaltene concentration increases. Assuming that the viscosity increases with the asphaltene concentration, at the same time the rotational dynamics of the macroaggregates becomes progressively slower. Similarly, the average distance $\langle d\rangle$ as well as the concentration layer thickness $\sqrt{ } a$ becomes very small because $\langle d\rangle \propto c_{a s p h}^{-}{ }^{1 / 3}$ (Fig. 18b). As shown above, we have an exponential concentration profile $u(x)$ in the proximity of the separated phases. One can thus assume a similar form for a viscosity gradient on a limited range of distance $x$ from the surface of the macro-aggregates that will influence the translational dynamics of hydrocarbons. For these reasons, the following $a$ priori exponential form has been introduced [114]:

$\tau_{1 D}=\frac{12 \pi R_{m o l}^{3} \eta}{k_{B} T}\left[1-\exp \left(-\eta / \eta_{c}\right)\right]$

showing that $\tau_{1 D}(\eta)$ tends to a constant value that is independent of the viscosity above a threshold viscosity $\eta_{c}$ and fulfils the StokesEinstein equation (Eq. 61b) below $\eta_{c}$ (Fig. 19). In other words, the dynamics of the highly confined hydrocarbons becomes similar to that for a wetting fluid close to a solid surface. For instance, this behaviour is encountered in the $2 \mathrm{D}$ correlation map of the translational diffusion constant, $D-T_{2}$ for water in porous sandstone rocks, where the observed $D \propto 1 / \tau_{1 D}$ is independent of $T_{2}$ over the whole distribution of the $T_{2}$ values [124-126]. The tortuosity of the diffusion in the transient porous medium (Fig. 18a) also favours this assumption. Fig. 19 shows the Stokes-Einstein variations of $\tau_{\text {rot }}$ and $\tau_{1 D}$ when the viscosity varies over a large range, $1 \leq \eta \leq 10^{6}$ $\mathrm{cP}$, combined with a threshold value for viscosity $\eta_{c} \sim 300 \mathrm{cP}$ obtained from Eq. (62). The validity of Eq. (62) will be demonstrated in the following section by a direct comparison with the viscosity dependencies of the observed relaxation times $T_{1}(\eta), T_{2}(\eta)$ of various crude oils $[113,127]$.

\subsubsection{Comparison with the observed viscosity dependencies of $T_{1}$ and} $\mathrm{T}_{2}$

This section aims to explain the universal relation observed between the log-mean averages $\left\langle T_{2, L M}\right\rangle,\left\langle T_{1, L M}\right\rangle$ and the viscosity $\eta$ and asphaltene concentration for a whole set of experimental data on various heavy crude oils (Fig. 15). Relaxation equations are shown [114] for the longitudinal and transverse nuclear relaxation rates constants $1 / T_{1}$ and $1 / T_{2}$ of the observed protons of saturated hydrocarbon chains diffusing among the clusters of asphaltenes containing the paramagnetic species $\mathrm{VO}^{2+}$ (Fig. 18). Consideration of all these experiments suggests a transition for the translational diffusion coefficient from the usual Stokes-Einstein relation $D \propto 1 / \eta$ which is conserved at low asphaltene concentrations below a threshold viscosity $\eta_{c}$ to a wetting behaviour where the diffusion coefficient does not vary with the viscosity close to the asphaltene aggregates at higher asphaltene concentration above $\eta_{c}$ [114] (Fig. 15). This model is consistent with the usually observed behaviours for crude oils in that $T_{2}(\eta)=T_{1}(\eta) \propto 1 / \eta$ for low viscosity, whereas at high asphaltene concentration and high viscosity one has $T_{2}(\eta) \propto 1 / \sqrt{ } \eta$ with a weak Larmor frequency dependence. In addition, $T_{1}$ becomes quasi-independent of the viscosity while it is proportional to the square root of the Larmor frequency $[113,127]$. The theory can also reproduce the general relaxation behaviours of the $2 \mathrm{D}$ correlation maps $T_{1}-T_{2}$ (Fig. 16) and $D-T_{2}$ (Fig. 17) for crude oils when varying the asphaltene concentration $[112,128]$. These results provide valuable tools for the down-hole NMR characterization of petroleum fluids. From the basic properties of the nuclear diffusion-relaxation model in a low dimensional system [71] with paramagnetic $\mathrm{VO}^{2+}(S=1 / 2)$ embedded in the asphaltene nano-aggregates, the spectral density function $\mathrm{J}_{1 \mathrm{D}}(\omega)$ describing the quasi 1D translational diffusion of these proton-hydrocarbon species $(I)$ in between the slowly rotating asphaltene macro-aggregates may be written as:
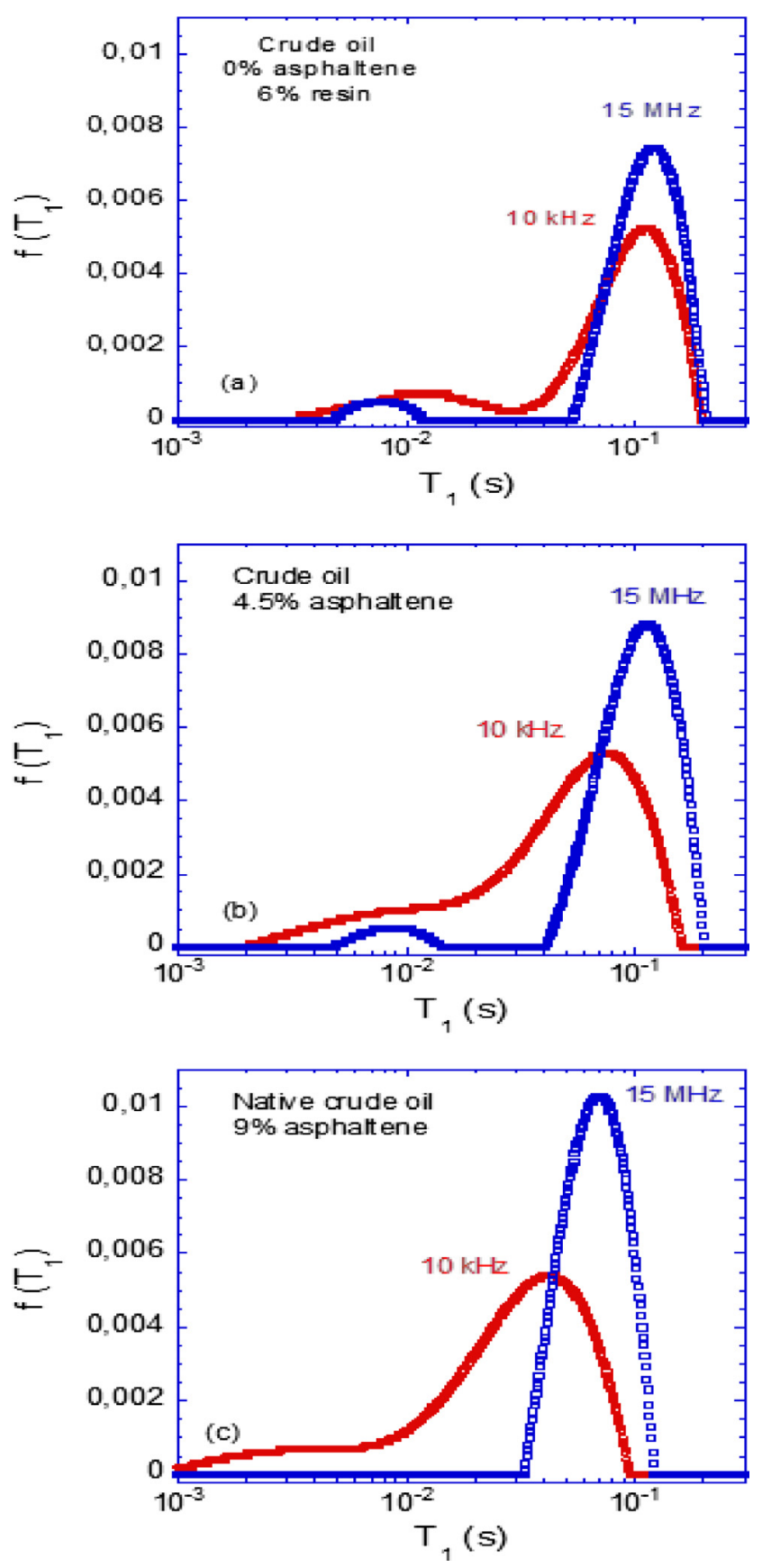

Fig. 21. One-dimensional $T_{1}$-distributions $f\left(T_{1}\right)$, measured at the lowest $(10 \mathrm{kHz})$ and highest Larmor frequencies $(15 \mathrm{MHz}$ ) obtained by inverse Laplace transform (ILT) of the longitudinal magnetization decays of crude oil samples without (a) and with 4.5\% (b) and 9\% (c) asphaltene. The data are taken from Fig. 9 of Ref. [110]. 

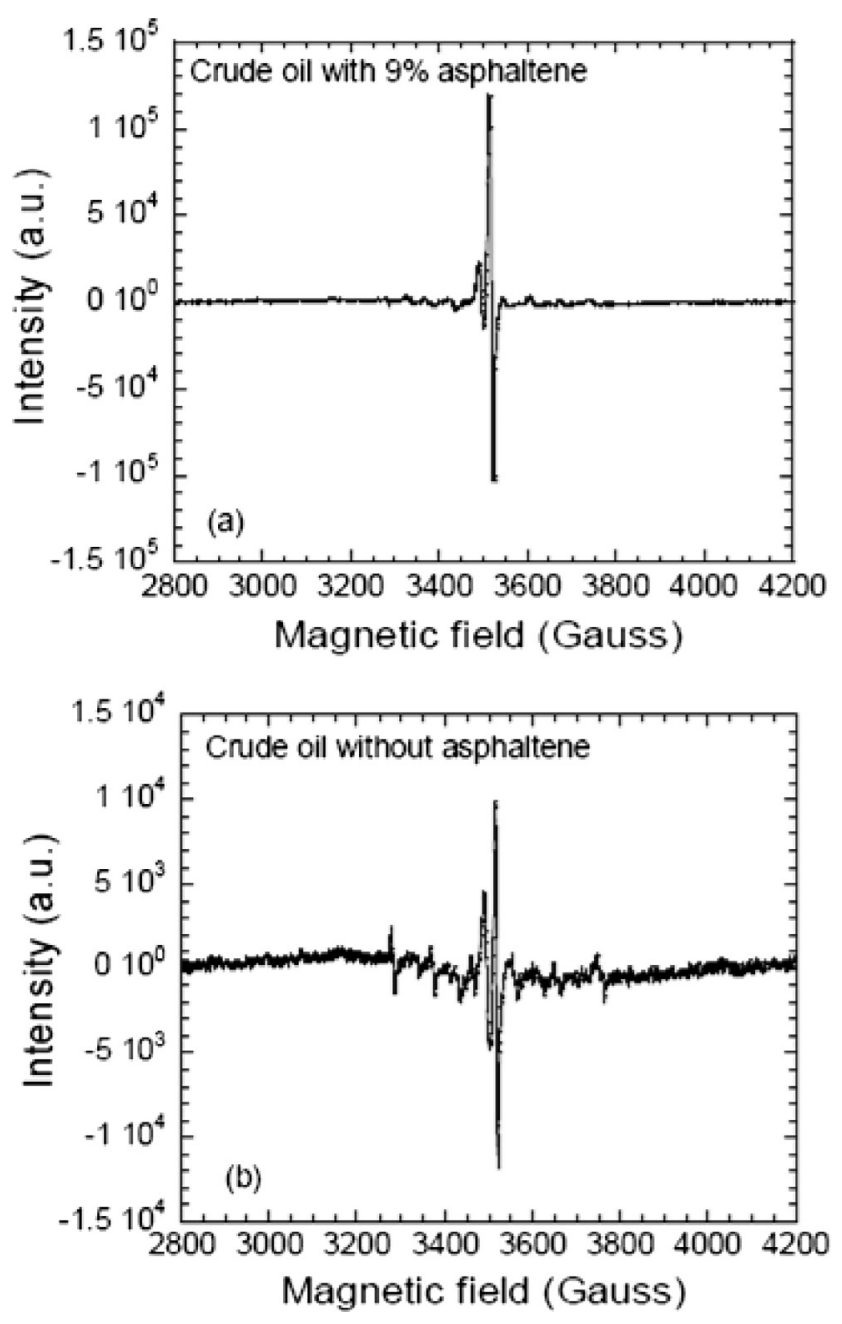

Fig. 22. (a) X-band ESR spectrum of a bulk crude oil with $9 \%$ asphaltene. (b) ESR spectrum of the same bulk crude oil but after removing the asphaltene. The data are taken from Fig. 3 of Ref. [112].
$J_{1 D}(\omega)=\sqrt{2 \pi} \sqrt{\tau_{1 D} \tau_{\text {rot }}} \frac{\sqrt{1+\sqrt{1+\omega^{2} \tau_{\text {rot }}^{2}}}}{\sqrt{1+\omega^{2} \tau_{\text {rot }}^{2}}}$.

Similar results have been obtained with another dynamical model of hydrocarbons within asphaltene aggregates in crude oils [129]. For a heteronuclear $(I-S)$ dipolar relaxation process mediated by $1 \mathrm{D}$ translational diffusion, $1 / T_{2}$ and $1 / T_{1}$ are proportional to the following linear combinations of the spectral densities at the nuclear $\left(\omega_{I}\right)$ and electronic $\left(\omega_{S}=659 \omega_{I}\right)$ Larmor frequencies:

$$
\begin{aligned}
\frac{1}{T_{2}\left(\omega_{I}\right)} & =2 A\left[J(0)+\frac{3}{4} J\left(\omega_{I}\right)+\frac{13}{4} J\left(\omega_{S}\right)\right] \\
& =\left(\frac{\mu_{0}}{4 \pi}\right)^{2} 4 \sqrt{\pi} A \sqrt{\tau_{1 D} \tau_{\text {rot }}}\left[1+\frac{3 \sqrt{2}}{8} F\left(\omega_{I}, \tau_{\text {rot }}\right)+\frac{13 \sqrt{2}}{8} F\left(\omega_{S}, \tau_{\text {rot }}\right)\right],
\end{aligned}
$$

$$
\begin{aligned}
\frac{1}{T_{1}\left(\omega_{I}\right)} & =A\left[3 J\left(\omega_{I}\right)+7 J\left(\omega_{S}\right)\right] \\
& =\left(\frac{\mu_{0}}{4 \pi}\right)^{2} \sqrt{2 \pi} A \sqrt{\tau_{1 D} \tau_{\text {rot }}}\left[3 F\left(\omega_{I}, \tau_{\text {rot }}\right)+7 F\left(\omega_{S}, \tau_{\text {rot }}\right)\right] .
\end{aligned}
$$

In Eqs. (64) and (65):

$$
\begin{aligned}
A & =\sigma_{S} S_{P, N M R} \varrho_{o i l}\left(\gamma_{I} \gamma_{S} \hbar\right)^{2} \delta_{I S}^{-3} S(S+1), F\left(\omega_{I}, \tau_{\text {rot }}\right) \\
& =\frac{\sqrt{1+\sqrt{1+\omega_{I}^{2} \tau_{\text {rot }}^{2}}}}{\sqrt{1+\omega_{I}^{2} \tau_{\text {rot }}^{2}}} .
\end{aligned}
$$

The surface density of paramagnetic species, $\sigma_{S}$, of spin $S=1 / 2$, which has been obtained from calibrated ESR measurements [112]. The quantity $S_{P, N M R}=S_{P} F$ is an NMR-based specific surface area [84] of the asphaltene aggregates that is proportional to the true specific surface area $S_{P}$ of asphaltene and the ratio $F=m_{\text {sol,eq }} / m_{\text {liq,eq }} \ll 1$ of the solid- to the liquid-proton magnetization at equilibrium. $\rho_{\text {oil }}$ is the crude oil density and $\left\langle\delta_{I S}\right\rangle$ represents the average distance of minimal approach between $I$ and $S$ spins in the local geometry of Fig. 18a. The correlation time $\tau_{\text {rot }}$ (Eq. (61a)) and $\tau_{1 D}$ (Eq. (61b)) have been introduced in Eqs. (64) and (65) for a direct comparison

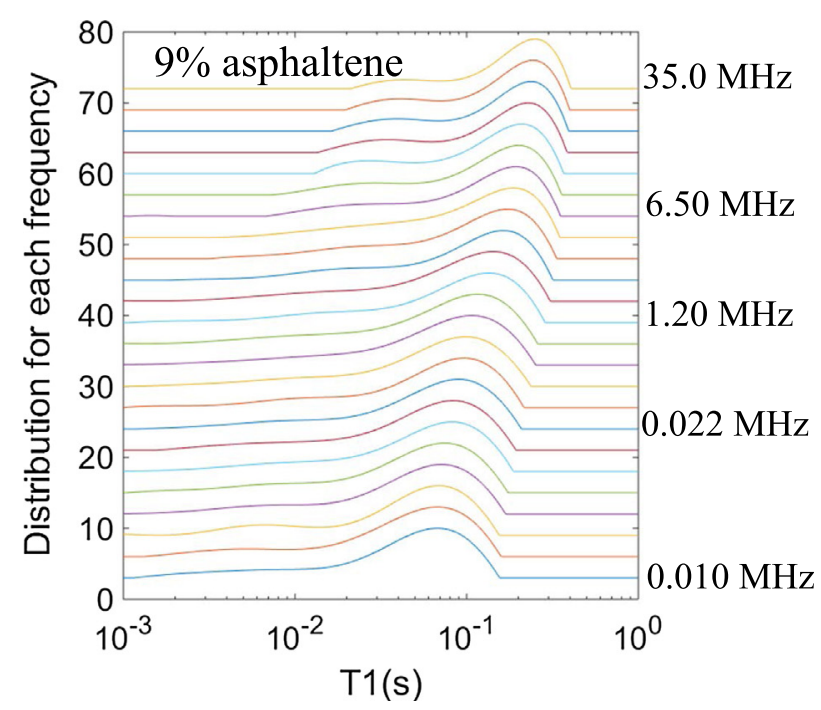

(a)

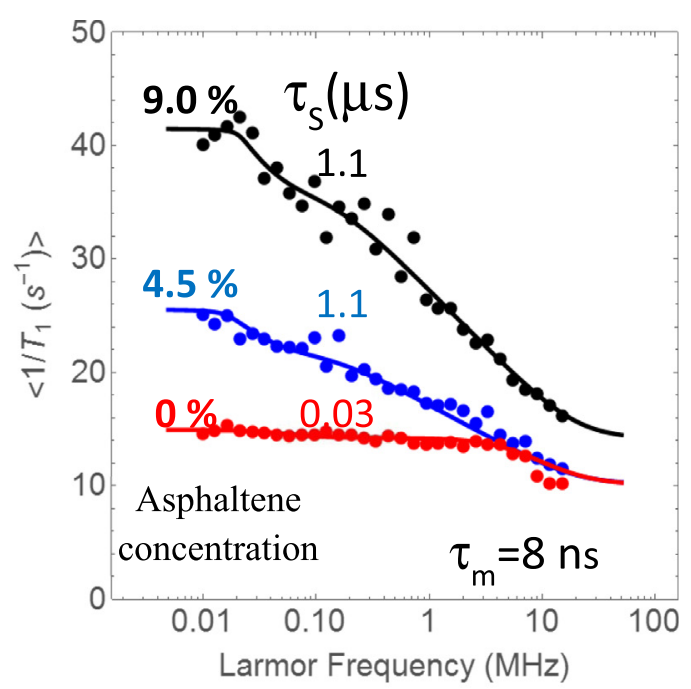

(b)

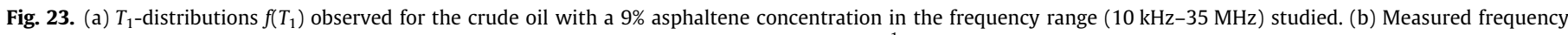

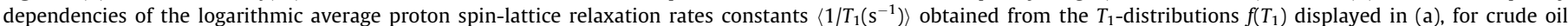

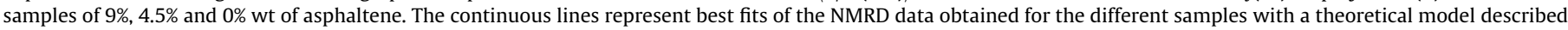
in Section 8.3 and Refs. [112,136]. 
with the viscosity dependence of experimental data of $\left\langle T_{2, L M}\right\rangle$ and $\left\langle T_{1, L M}\right\rangle$ measured at $2.5,23$ and $80 \mathrm{MHz}$ for a large set of different alkanes and crude-oils at room temperature by Korb et al. $[113,127]$. Though there is an extreme diversity in the experimental oil data, a comparison amongst them was facilitated by choosing a set of common parameters as follows: oil density, $\rho_{\text {oil }}=0.85 \mathrm{~g} / \mathrm{cm}^{3} ;$ minimal approach distance between $I$ and $S$ spins, $\left\langle\delta_{I S}\right\rangle=0.655 \mathrm{~nm}$ for $T_{1}$ and $0.855 \mathrm{~nm}$ for $T_{2}$ (corresponding to the average molecular sizes of a $\mathrm{C} 8-\mathrm{C} 12$ species); cross-over for viscosity, $\eta_{C}=250 \mathrm{cP}$ for $T_{2}$ and $160 \mathrm{cP}$ for $T_{1}$ (except for $T_{1}$ at $2 \mathrm{MHz}$ with $\eta_{C}=3500 \mathrm{cP}$, where the cross-over viscosity $\eta_{C}$ is enhanced at low frequency); and $S_{P, N M R}=S_{P} F \sim 0.86 \mathrm{~m}^{2} / \mathrm{g}$ when $F=1 / 100$. Previous quantitative ESR spectra of crude oil found a surface density of $\sigma_{S}=1.7 \times 10^{13} \mathrm{VO}^{2+} / \mathrm{cm}^{2}$ with spins $S=1 / 2$ [125], which corresponds to an average distance between two $S$-spins within asphaltene aggregates of $1 / \sqrt{ } \sigma_{S} \sim 2.55 \mathrm{~nm}$, of the order of the average radius of a macro-aggregate. Finally, $R_{\text {agg }}=$ $2.6 \mathrm{~nm}$ and $R_{m o l}=0.39 \mathrm{~nm}$ correspond to C8-C12 chain lengths. The good agreement between experiments and theory over a very large range of viscosities, shown in Fig. 15, demonstrates the following expected behaviours: $T_{2}(\eta)=T_{1}(\eta) \propto 1 / \eta$ for low viscosity and $T_{2}(\eta) \propto 1 / \sqrt{ } \eta$ and the viscosity independence and strong Larmor frequency dependence of $T_{1}(\eta) \propto \sqrt{\omega_{0} / \tau_{1 D}} \approx C^{t e}$ for high viscosity. This agreement validates Eqs. (64) and (65) and shows that the data contain more than just correlations relating NMR relaxation times and viscosity.

\subsubsection{Comparison between observed $2 D$ correlation maps $T_{1}-T_{2}$ for different crude oils}

Fig. 16 shows an example of $T_{1}-T_{2}$ data obtained at $2.5 \mathrm{MHz}$ for a native crude oil with a concentration of $9 \mathrm{wt} \%$ asphaltene and $6 \mathrm{wt}$ \% resin [114]. The continuous lines in Fig. 16 represent the $2 D T_{1}-T_{2}$ data calculated using the parametric Eqs. (64) and (65) for different values of $R_{\text {agg, }}$, and with the form of $\tau_{\text {rot }}(\eta)$ and $\tau_{1 D}(\eta)$ proposed in Eqs. (61a) and (62). As expected, when $R_{\text {agg }}$ decreases in the range $R_{\text {agg }} \in(1.0,1.5,2.0$ and $2.5 \mathrm{~nm})$, the $2 D T_{1}-T_{2}$ data becomes progressively closer to the $T_{1}=T_{2}$ line. The best agreement with experiment is obtained for $R_{\text {agg }}=2.6 \mathrm{~nm}$, justifying again the hypothesis that the relaxation data reflect a 1D translational diffusion of hydrocarbon chains between the asphaltene macro-aggregates (Fig. 18); essentially, the theory reproduces the main features of the relaxation experiments. (i) For long relaxation times $T_{1}$ and $T_{2}$, one has $T_{1} \sim T_{2}(\propto 1 / \eta)$, which corresponds to the relaxation induced by fast reorientational dynamics of small hydrocarbon chains. (ii) For short relaxation times $T_{1}$ and $T_{2}$, there is a systematic upward trend away from the line $T_{1}=T_{2}$ that reproduces the theoretical predictions $T_{2}(\eta) \propto 1 / \sqrt{ } \eta$ and $T_{1}(\eta) \approx C^{t e}$, consistent with slow translational diffusion of the long hydrocarbon chains between slowly rotating asphaltene macro-aggregates.

\subsubsection{Comparison with the observed $2 D$ correlation maps $D-T_{2}$ for crude oils with asphaltene}

The main interest in using the 2D NMR spin correlation $D-T_{2}$ is to probe molecular dynamics on different length scales. The diffusion editing PGSE sequence probes the translational diffusion in the micrometer range using a diffusion delay of $\Delta=24 \mathrm{~ms}$, while the CPMG $T_{2}$ sequence with short inter-pulse delays explores the molecular dynamics only on the nanometer range [128]. In this section, the anomalous features of the $D-T_{2}$ data in crude oils for various asphaltene concentrations are interpreted with the relaxation theory described in Section 8.1.2. Because the translational diffusion coefficient is inversely proportional to the translational correlation time $D_{1 D} \propto 1 / \tau_{1 D}$, Eq. (62) leads to the following form: $D_{1 D}(\eta)=k_{B} T /\left\{6 \pi R_{\text {mol }} \eta_{c}\left[1-\exp \left(-\eta / \eta_{c}\right)\right]\right\}$ by taking into account the transition from the Stokes-Einstein relation $D_{1 D} \propto 1 / \eta$ to a constant value $D_{1 D} \approx$ Cte above the threshold viscosity $\eta_{c}$. Fig. 17a shows the superposition of $D-T_{2}$ data obtained at 2.5 $\mathrm{MHz}$ for a crude oil diluted by its own maltene (saturated, aromatic and resin components other than asphaltene are defined as maltenes) at three different asphaltene concentrations ranging between $0 \%$ wt (pure maltene) and 9\% wt (native crude oil) [114]. When the asphaltene concentration $c_{a s p h}$ increases, there is a systematic shift of the whole $D-T_{2}$ data towards short $T_{2}$ values and away from the usual linear $D_{1 D} \propto T_{2}$ relation as the $D$ values level off. This behaviour has been observed previously see Fig. 4 of Ref. [128]
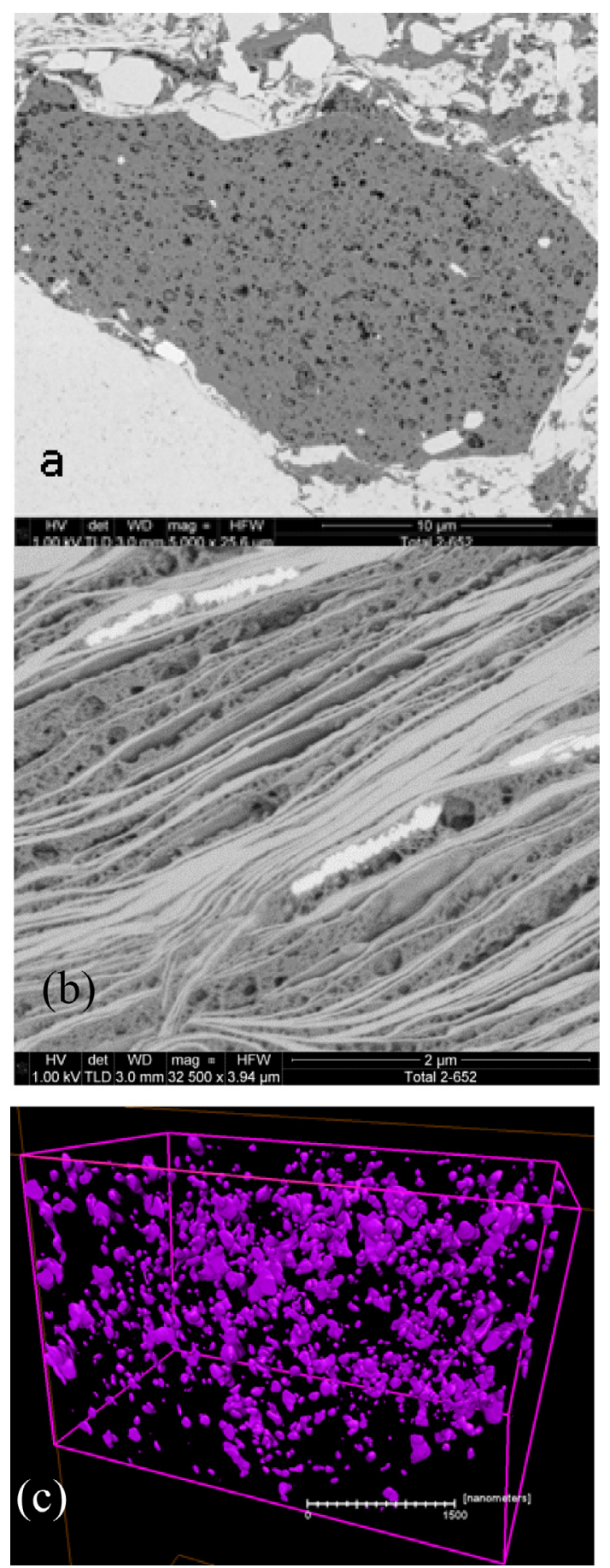

Fig. 24. Scanning electron micrographs of the organic kerogen (a) and mineral materials (b) measured in an shale oil sample [22]. (c) FIB-SEM micrograph showing the microporous structure of the kerogen in a shale oil sample. The data come from Ref. [140] 

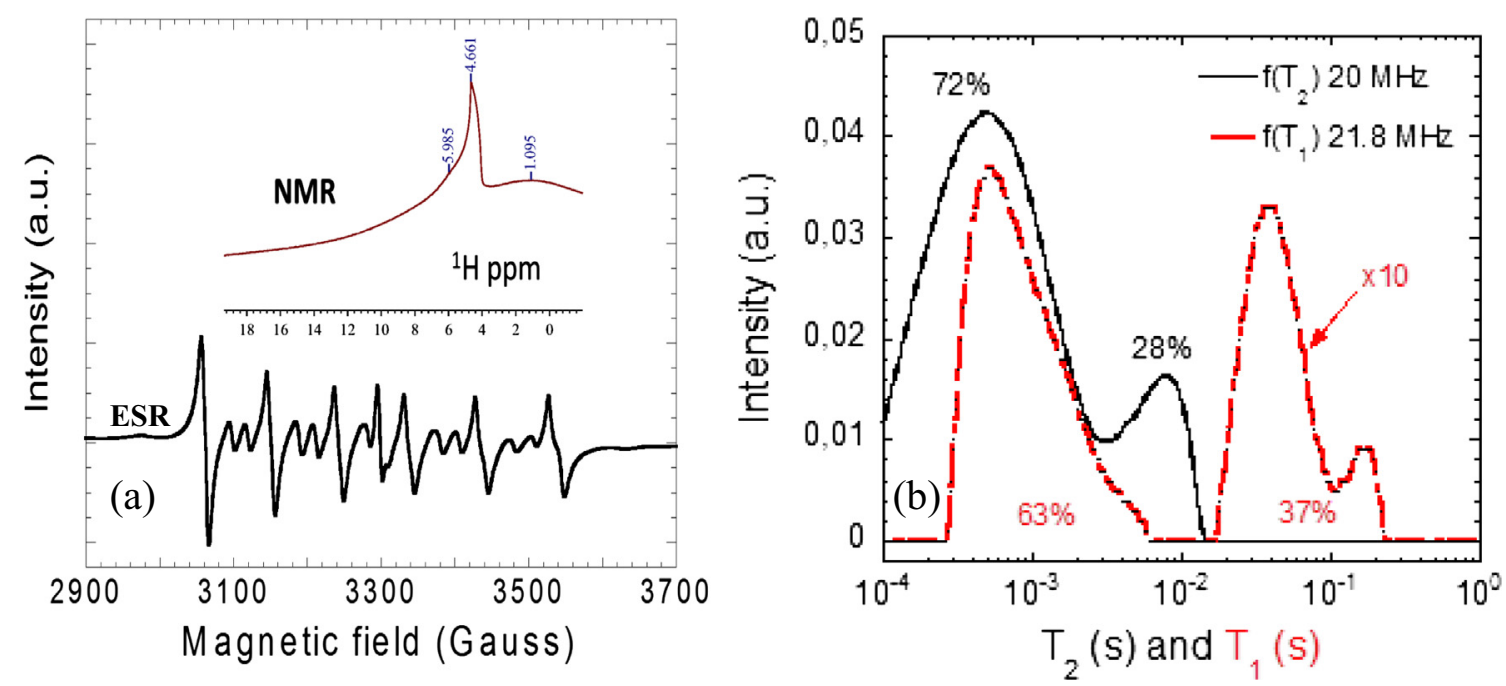

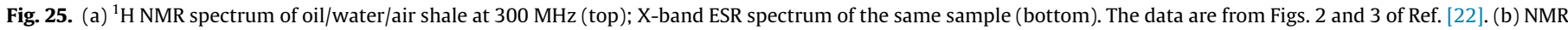

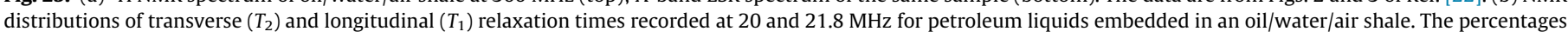
indicate the relative enclosed area of each peak. The second peak intensity at high $T_{1}$ and $T_{2}$ values has been scaled by a factor 10 .

and indicates an enhancement of the surface wettability of the crude oil macro-aggregates. Fig. 17b shows experimental data obtained at 2.5 and at $23 \mathrm{MHz}$ for the $9 \%$ wt asphaltene (native crude oil). There is a better signal to noise ratio for the $23 \mathrm{MHz}$ data, and the leveling off of the diffusion coefficient $D$ at short $T_{2}$ values is clear (Fig. 17b). To reproduce the experimental data of Fig. 17a and b, the form of $D_{1 D}(\eta)$ given above was correlated with a rescaled $T_{2, \text { resc }}\left(\eta, \eta_{c}\right)$ obtained by multiplying Eq. (64) by the pseudo porosity $\Phi\left(c_{a s p h}\right)$ introduced in Fig. $18 \mathrm{~b}$. This rescaling of the $T_{2}$ values is necessary to take into account the observed shift of the $D-T_{2}$ data towards shorter $T_{2}$ values with increasing asphaltene concentration. These calculated $D-T_{2, \text { resc }}$ data obtained for $\eta_{c} \in$ [5-80 cP] reproduce the main experimental features: (i) In the low asphaltene concentration domain, one obtains the usual behaviour $D_{1 D} \propto T_{2} \propto 1 / \eta$ typical of the Stokes-Einstein relation with a threshold viscosity $\eta_{c}$ reaching the highest values in the range of viscosity studied [110]. (ii) When increasing the asphaltene concentration, the calculated data (continuous lines in Fig. 17a and b) reproduce the corresponding experiments as the threshold viscosity $\eta_{c}$ decreases. (iii) The diffusion coefficients $D$ as well as the $T_{2}$ values agree with the experimental values. (iv) The transition from the usual relation $D \propto T_{2}$ to the anomalous relation $D \propto \sqrt{T_{2}}$ occurs in a very limited range of short $T_{2}$ values. Even in the experimental data, it is not clear whether this transition exists rather than a leveling off $\left(D \sim C^{t e}\right)$ that better agrees with the observed data for short $T_{2}$ values (Fig. 17). Unfortunately, experimental $D-T_{2}$ data for values of $T_{2}<10 \mathrm{~ms}$ are unavailable due to the diffusion editing step that is used prior to the CPMG $T_{2}$ sequence. The good agreement between the aforementioned theory and the $D-T_{2}$ experiments performed for different asphaltene concentrations is shown more clearly at $23 \mathrm{MHz}$ (Fig. 17b). The data observed at $2.5 \mathrm{MHz}$ and $23 \mathrm{MHz}$ represent an important issue since the $D-T_{2}$ correlation can be used down-hole allowing an in situ quantitative characterization of the crude oil wettability and asphaltene concentration.

\subsection{Dynamic surface affinities of diphasic liquids as a probe of wettability of multimodal macroporous petroleum rocks}

The wettability of a rock/oil/brine system affects fluid saturation, capillary pressures, electrical properties and relative permeabilities [130-133]. Wettability by fluids in oil and gas reservoirs has a direct effect on efficiency of hydrocarbon recovery. For oil recovery, water injection is generally used to move oil towards production zones, and if wettability variations occur, the water has the tendency to flow predominantly within the water-wet locations, leaving oil in place in oil-wet locations. Because of its economic impact, in situ control and monitoring of wettability justify huge investments in core analysis for laboratory measurements.

Techniques that are able to probe wettability in the field are still not fully developed. Measurements on cores require long and tedious preparation, and never reflect the actual state of wettability because the rock cores are generally not tested in their native state; they are always cleaned and saturated with known brine and oil fluids, and are aged and tested in conditions of spontaneous drainage (oil in water saturated core) and imbibition (water in oil saturated core). Two wettability measurement methods (Amott and USBM) are effectively accepted as standards by industry; however they are not strictly comparable in all cases. The Amott index [133] is defined as running from 1 for water-wet rocks to -1 for oil-wet cores. It is obtained from a special core analysis using a centrifuge that combines two spontaneous imbibition measurements and two forced displacement measurements resulting in a plot of the capillary pressure curve as a function of the water saturation. An alternative method called USBM (U.S. Bureau of Mines) uses a pressure gradient to force oil flow in a water-saturated core and reciprocally to force water flow in an oil-saturated core. The USBM index (varying between $-\infty$ and $+\infty$ ) is defined as the logarithm of the ratio of the energy required to move oil with water vs the energy required to move water with oil. A recently published NMR-based method introduced an index defined as the total surface of rock wetted by water minus the total surface of rock wetted by oil, all divided by the total surface in contact with fluid (i.e. water or oil) [134,135]. This method uses the sharp difference of $T_{2}$ distribution of a bulk and transient surface fluid in pores. However, the method requires a measurement at complete water saturation and the knowledge of the $T_{2}$ distribution of the oil in bulk conditions. None of these techniques provide local probes of interaction between the fluid and the rock-pore surface in a single non-invasive measurement.

To overcome this limitation, Korb et al. proposed using NMRD measurements to probe the wettability of carbonate rocks [27]. Fig. 20 shows examples of data from carbonate rocks from a Middle 

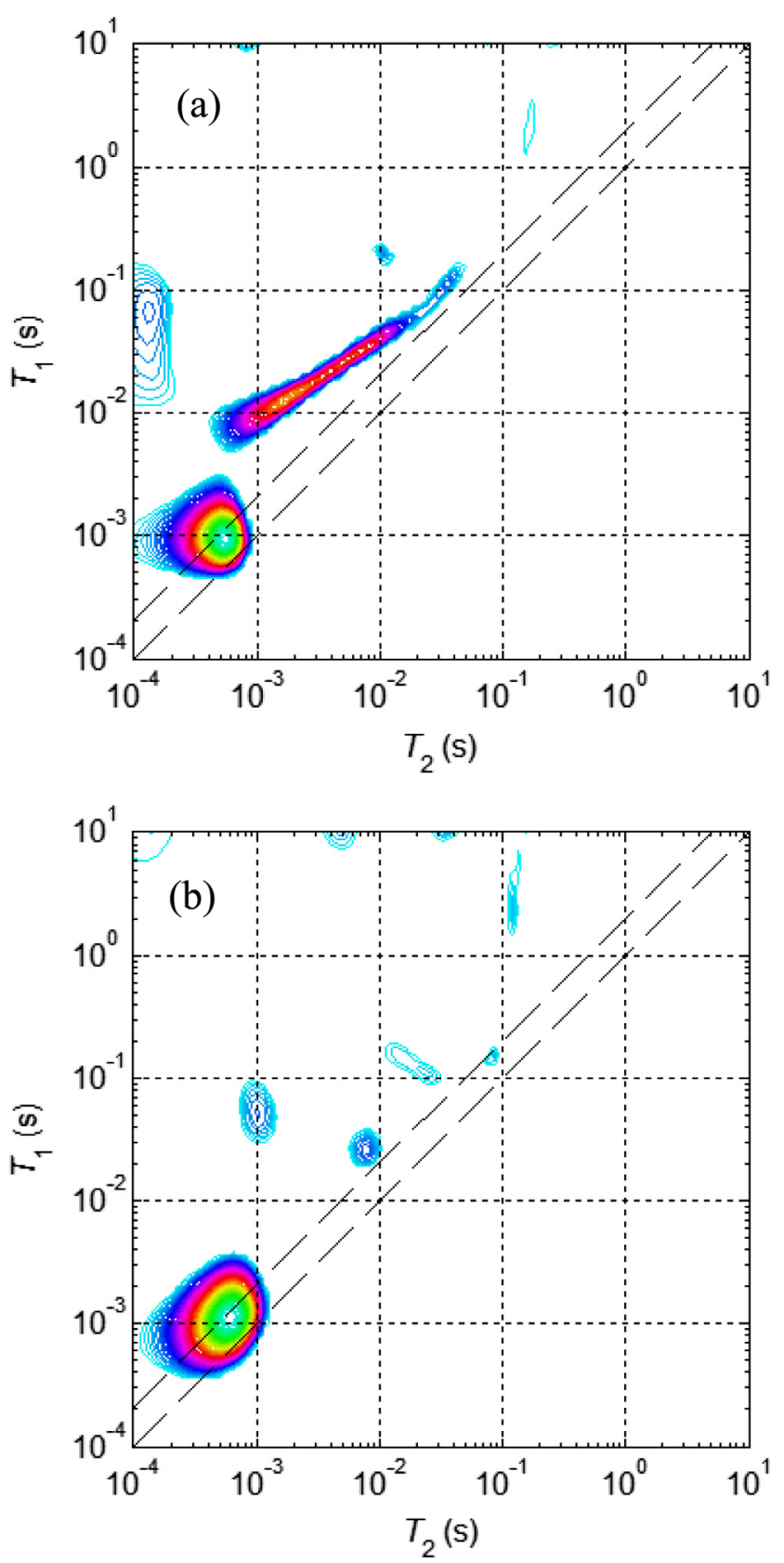

Fig. 26. Two-dimensional $T_{1}-T_{2}$ spin-correlation maps of untreated oil/water/air (a) and water/air (b) shales, recorded at $2.5 \mathrm{MHz}$ and $35^{\circ} \mathrm{C}$. The data are taken from Ref. [22]. A color code scale is given for estimating the relative intensities of the different peaks. The dashed lines correspond to $T_{1}=T_{2}$ and $T_{1}=2 T_{2}$.

Eastern oil reservoir (these are intraclast skeletal ooid grainstone carbonates with $30 \%$ porosity and $700 \mathrm{mD}$ permeability; their dual porosity was shown by the bimodal NMR $T_{1}$ distribution obtained after saturation with brine) [27]. After cleaning, filling and drying, rock plugs corresponding to three different conditions of saturation were prepared and analysed by NMRD: (i) 100\% dodecane saturation $\left(S_{w}=0 \%\right)$ : this plug was vacuumed and saturated with dodecane and taken to be at $0 \%$ water saturation (Fig. 20a). (ii) $100 \%$ water saturation $\left(S_{w}=100 \%\right.$, Fig. 20b): this plug was vacuumed and saturated with $50 \mathrm{kppm} \mathrm{NaCl}$ brine (use of brine rather than fresh water prevented dissolution of the rock). (iii) Irreducible water saturation $S_{\text {wirr }}$ : this plug was first saturated $100 \%$ with brine and then dodecane is forced into the plug by high speed centrifugation (Fig. 20c). Fig. 20a-c shows two different NMRD profiles
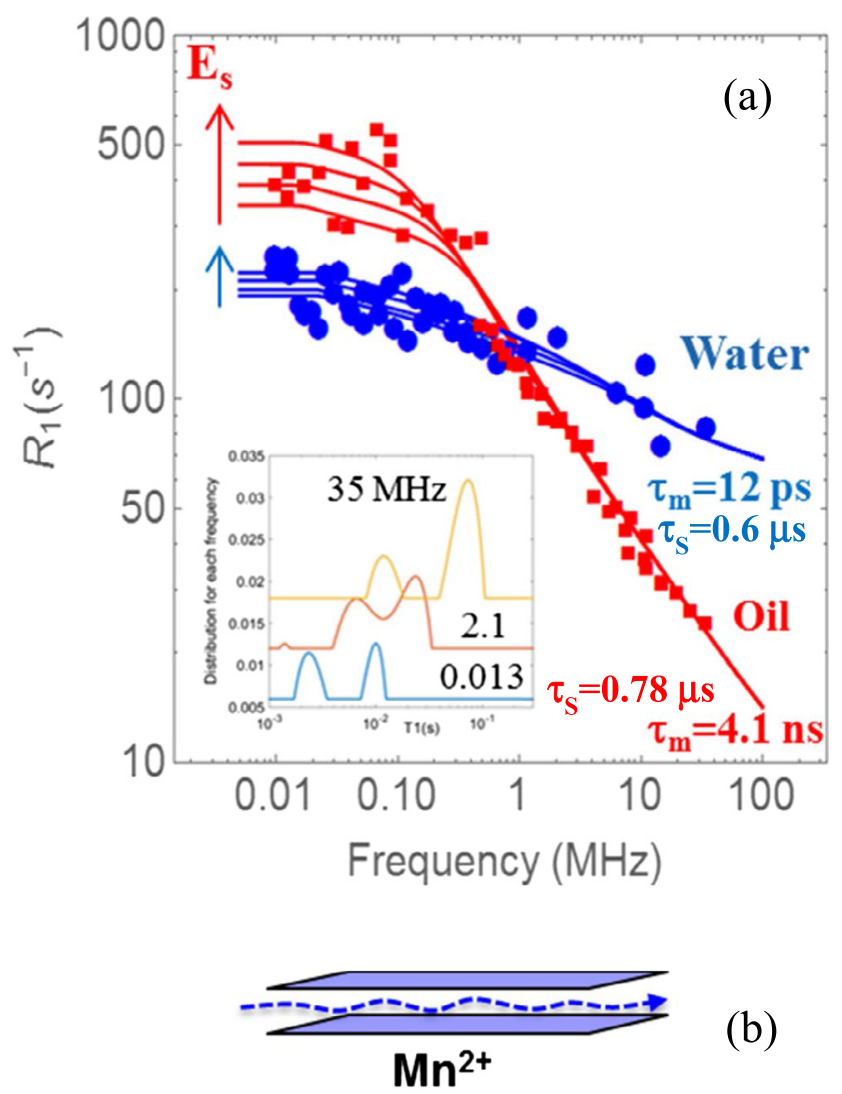

(b)

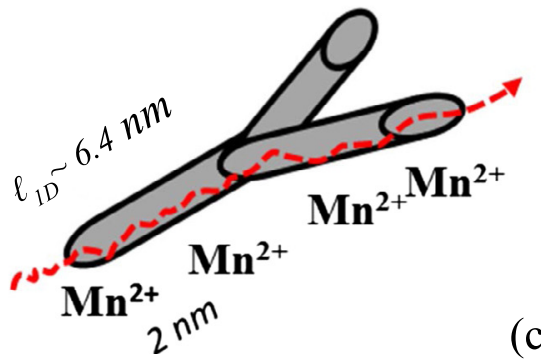

(c)

Fig. 27. (a) Logarithmic plot of the measured proton spin-lattice relaxation rate constant $R_{1}$ as a function of the proton Larmor frequency for an oil/brine/air shale (square and circle points) [140]. The method described in the text was used to obtain separated water and oil NMRD profiles. The continuous blue (water) and red (oil) lines are best fits obtained using Eqs. (67) and (70) respectively, with the values obtained for $\tau_{m}$ and $\tau_{S}$ shown on the figure. The activation energy $E_{s}$ at low frequency was varied (see text). (b) Schematic diagram showing 2D water diffusion in the proximity of $\mathrm{Mn}^{2+}$ ions at clay pore surfaces. (c) Schematic diagram showing the quasi-1D oil diffusion in proximity to the paramagnetic species at kerogen pore surfaces.

made from the two peak values of the bimodal distributions (an example is given at $15 \mathrm{MHz}$ in Fig. 20d) obtained for all the frequencies $(10 \mathrm{kHz}-20 \mathrm{MHz})$ studied. The two associated relaxation rates labeled $R_{11}=1 / T_{11}$ and $R_{12}=1 / T_{12}$ are proportional to the macropores and micropores, respectively. The NMRD data obtained in the case of a monophasic saturation of carbonate rocks with dodecane present a bi-logarithmic frequency dependences for $R_{11}=1 / T_{11}$ and $R_{12}=1 / T_{12}$ (Fig. 20a). The rescaling of the two rates separated by a factor 4 data onto a single bi-logarithmic master curve (inset of Fig. 20a) proves unambiguously the existence of a unique intermolecular dipolar relaxation mechanism modulated by translational 2D diffusion of dodecane molecules at the vicinity of paramagnetic impurities $\left(\mathrm{Mn}^{2+}\right.$ ions) at the pore surface, as 
described quantitatively by Eq. (55). As described in Section 6.2, this model introduced two correlation times: (i) the translational correlation time $\tau_{m}$ associated with individual molecular jumps in proximity to the surface. (ii) the surface residence time $\tau_{s}$, that describes how long the proton species $I$ and the manganese stay dynamically correlated. This time $\tau_{s}$, depends on both the strength of the surface interactions and the reoccurrence of first neighbour interactions induced by the fluid confinement in pores. The introduction of a dynamical surface affinity index: $A=\tau_{S} / \tau_{m}$ has proven useful for estimating the microscopic wettability [27]. This index $A$ represents roughly the average number of diffusing steps of spins $I$ in the vicinity of fixed paramagnetic sites $S$ during the time scale of a NMRD measurement. The larger this index, the more numerous are the $2 \mathrm{D}$ re-encounters and therefore the more correlated are the movements of $I$ and $S$ spins. Its value ranges between 1 for the bulk case and $\infty$ for the case where the moving protons have highly correlated two-dimensional dynamics in the proximity of the fixed paramagnetic sources at the pore surfaces. Thus $A$ reveals the affinity of the fluid for the pore surface, i.e. it is the NMRderived microscopic wettability. The fit of Fig. 20a with Eq. (55) gives: $\tau_{m}=1 \mathrm{~ns}$ and $\tau_{s}=130 \mathrm{~ns}$, leading to a weak surface dynamical affinity $A=130$ for oil in this well-known water-wet rock. Owing to the constant value of the affinity ratio, $R_{12}\left(\omega_{I}\right) /$ $R_{11}\left(\omega_{I}\right) \sim 8$, within the whole frequency range studied, a rescaling of the NMRD data is shown in the inset of Fig. 20b. The particular form of this NMRD profile proves that the relaxation process is due to the nuclear paramagnetic relaxation of water molecules interacting with metallic paramagnetic ions at the pore surfaces, since the frequency dependence of $1 / T_{1}$ is the sum of two Lorentzian spectral densities at nuclear $\omega_{I}$ and electronic $\omega_{S} \sim 659 \omega_{I}$ Larmor frequencies (see the 2nd part of Eq. (56)).

Last, the NMRD data obtained in the case of a diphasic mixture saturating the carbonate rocks with both dodecane and brine is shown in Fig. 20c. The relaxation rates of the mixture (large full dots) are compared with the rates $R_{11}$ and $R_{12}$ (empty dots) obtained with monophasic saturations of dodecane $\left(S_{w}=0 \%\right.$, data taken from Fig. 20a) and water $\left(S_{w}=100 \%\right.$, data taken from Fig. 20b), respectively. Here, $R_{11}$ has the typical NMRD signature of dodecane in large pores leading to $A=130$ (Fig. 20a), whereas $R_{12}$ exhibits the typical NMRD signature of brine in small pores (Fig. 20b), thus establishing that water fills and wets the small pores while dodecane is located in the macro-porosity, but is only weakly wetting. Given that in this example the cores were saturated with water first, this is the expected result, but in cases where there is no a priori knowledge of preferred wettability, this approach should allow identification of the wetting fluid for each pore type. At each frequency, a bimodal $T_{1}$ distribution is obtained (Fig. 20d), and the typical forms of the NMRD relaxation profiles for water and dodecane in confinement (Fig. 20c) allow a clear identification of the liquid present here. Integrating the distribution displayed in Fig. 20d thus gives an irreducible water saturation $S_{\text {wirr }}=20 \%$ for the whole frequency range studied (inset of Fig. 20d).

\subsection{Structure and dynamics of crude oils in bulk}

NMRD studies have also been useful in investigating the structure and dynamics of crude oils in bulk (taken largely from Vorapalawut et al. [110]). Fig. 21a-c shows the $T_{1}$-distributions $f\left(T_{1}\right)$ of crude oils containing asphaltene concentrations of $9 \% \mathrm{wt}$ (native), $4.55 \%$ wt or $0 \%$ (i.e. pure maltene), at the lowest (10 $\mathrm{kHz}$ ) and highest frequencies (15 MHz) available on the standard FFC spectrometer. The bimodal behaviour discussed in previous sections is more pronounced in presence of asphaltene and at the lower frequency, and is similar to distributions found by gas chromatography and gel permeation chromatography measured previously [136], showing that the hydrocarbon dynamics is directly related to the chain length. ESR measurements have been used for characterizing the different paramagnetic species in crude oils. X-band ESR spectra are shown in Fig. 22 for crude oils with (Fig. 22a) and without (Fig. 22b) asphaltene [112]. The paramagnetic $\mathrm{VO}^{2+}$ species can be unambiguously identified by the hyperfine interaction between the electron spin $S=1 / 2$ delocalized in the aromatic $\pi$ orbital and the nucleus of $V^{51}(I=7 / 2)$. Organic free radicals are also present in the oil fraction, but their nature is poorly understood due to the multiplicity of molecular structures that causes the unresolved and very broad ESR single line clearly observed in Fig. 22. Essentially, these ESR spectra are similar to that previously reported by Guedes et al. [137]. Comparison of Fig. 22a and b reveals a decrease of $63 \%$ between the quantities of paramagnetic impurities for the crude oil with and without asphaltene (intensity values of $2.38 \times 10^{8}$ with and $1.46 \times 10^{8}$ without asphaltene). However, one still observes $\mathrm{VO}^{2+}$ paramagnetic ions even after the asphaltene has been removed (Fig. 22b), demonstrating that part of the $\mathrm{VO}^{2+}$ is not trapped in asphaltene nano-aggregates but is still in bulk fraction of the crude oils. For several crude oils, pulsed ESR spectroscopy has shown the vanadium (IV) in the asphaltene is present in a porphyrinic structure, as there is coupling to four neighbouring nitrogens [138].

Fig. 23a shows the $T_{1}$-distributions $f\left(T_{1}\right)$ observed for the crude oil with a $9 \%$ asphaltene concentration in the frequency range (10 $\mathrm{kHz}-35 \mathrm{MHz}$ ) studied. Due to the observation of very large $T_{1}$ distributions, it is absolutely necessary to choose the log-average $\left\langle 1 / T_{1}\right\rangle$, defined in Section 8.1, for making the NMRD profiles displayed in Fig. 23b. This log-average represents a general method for analysing the large distribution $f\left(T_{1}\right)$ usually encountered in the petroleum industry. Fig. 23b shows the remarkable features of the log-average $\left\langle 1 / T_{1}\right\rangle$, for native crude oil (containing $9 \% \mathrm{wt}$ asphaltene), and samples containing $4.55 \%$ wt asphaltene (obtained by dilution of the native crude oil with maltene) or $0 \%$ asphaltene (i.e. pure maltene) [110]. The continuous lines in Fig. 23b represent the best fits of the NMRD data obtained [110] for the different samples with a theoretical model proposed in Refs. $[112,136]$ using a relaxation model (Eqs. (4)-(6) of Ref. [136]) due to intermittent surface dynamics of proton species in the proximity of the asphaltene nano-aggregate surface and bulk dynamics between the clusters of these nano-aggregates. The time of residence, $\tau_{S}=1.1 \mu \mathrm{s}$, and the 2D translational diffusion correlation time, $\tau_{m}=8 \mathrm{~ns}$ are found to be independent of the asphaltene concentration while the specific surface area $S_{P, N M R}$ increases linearly with asphaltene concentration. In fact, the used relaxation model considers the translational diffusion of hydrocarbons within the network geometry displayed in Fig. 18a assuming that all the 9\% (wt) asphaltene is incorporated into approximately $30 \mathrm{kDa}$ molecular mass. The mean interparticle separation is found to be around $7.3 \mathrm{~nm}$ (Fig. 18b), which is of the order of the particle diameter $(\approx 5.2 \mathrm{~nm})$ found by Mullins et al. [115] and gives for the native crude oil a 2D translational diffusion correlation time of $\tau_{m} \approx 8$ ns and residence time of $\tau_{S}=1.1 \mu \mathrm{s}\left(\tau_{S} \gg \tau_{m}\right)$, for the small hydrocarbons (octane) in close proximity to the asphaltene nanoaggregates. Given that the surface diffusion of the hydrocarbons close to the asphaltene nano-aggregates is 5.3 times smaller than the bulk diffusion, as shown by 2D NMR DOSY experiments [110], and given a molecular size $\delta_{\text {oil }}=0.655 \mathrm{~nm}$ for the oil (octane), this latter time $\tau_{S}$ gives an average radius of exploration for 2D hydrocarbon diffusion, $r_{2 D}=\sqrt{\frac{D_{\text {surf }}}{D_{\text {bulk }}} \frac{\tau_{s}}{\tau_{m}}} \delta_{\text {oil }} \approx 3.48 \mathrm{~nm}$; these results are of the same order of magnitude as those found by Eyssautier et al. with SAXS and SANS [109] of asphaltene solutions and by Mullins et al., at higher temperatures, by the observation of gravitational gradients of asphaltenes in oil field reservoirs [115].

The very long residence time found $\left(\tau_{S} \gg \tau_{m}\right)$ proves there is efficient interaction between maltene and asphaltene, and agrees 
with the theoretical model of anisotropic 2D diffusion of hydrocarbons in proximity to the asphaltene nano-aggregates having very slow rotation on such a time scale [136]. A complementary study of fluorine-containing tracer molecules in natural oils of different asphaltene content was proposed by Stapf et al. for proving the selective maltene-asphaltene interaction by analysing frequencydependent relaxation results [139]. For the crude oil largely without asphaltene (i.e. without any asphaltene aggregates), the situation is much simpler, leading to a value of $\tau_{s}=0.03 \mu$ s that is consistent with the absence of asphaltene. In that case, the asphaltene macro-aggregates disappear and even the nano-aggregates become very rare. The NMRD profile of such a sample with $0 \%$ wt asphaltene shown in Fig. 23 has almost no Larmor frequency dependence. This behaviour is typical of a fast hydrocarbon reorientational intramolecular relaxation process for the whole distribution of hydrocarbons. The continuous line displayed for this crude oil in Fig. 23 was obtained with the same translational correlation time $\tau_{m}=8 \mathrm{~ns}$ as for the native crude oil sample but with a very short residence time $\tau_{S}=30 \mathrm{~ns}$ that reflects the near-absence of any $2 \mathrm{D}$ diffusion. In the limit where $\tau_{S}$ is comparable to $\tau_{m}$, the frequency dependence of Eq. (6) of Ref. [136]) tends to Eq. (55) which almost disappears and the proposed theoretical 2D model tends to the usual 3D model for bulk relaxation.

\subsection{Dynamics and wettability of oil and water in the dual organic and mineral porosities of shale oils}

An accurate petrophysical evaluation of organic shales rocks is nowadays a challenge in the oil industry because liquid and gas hydrocarbons can be produced from these organic and mineral sedimentary rocks. This evaluation generally requires measuring the porosity, permeability and hydrocarbon saturation as well as dynamics and wettability of oil, water and gas trapped in the complex microstructure of these shale-oil rocks. However, most of the usual techniques cannot separate these fluids in the complex microstructure of shale rocks. Here again, NMRD and $T_{1}-T_{2}$ techniques have proven useful for probing in situ dynamics and wettability of these two fluids on these microporous rocks [22]. However, owing to the presence of two different fluids (oil and brine) embedded in the multiscale structures of organic (kerogen) and mineral (clay) pores, we need to outline in the following sections the necessary theoretical models for interpreting the NMRD and $T_{1}-T_{2}$ data.

\subsection{1. $T_{1}-T_{2}$ correlation and NMRD experiments with shale-oil rocks}

Details of sample preparation, characterization by scanning electron microscopy (SEM), ESR and NMR used can be found in Ref. [22]. SEM micrographs were used to characterize nanoporous organic kerogen (Fig. 24a) and mesoporous lamellar mineral samples (Fig. 24b), while focussed ion beam (FIB) SEM images clearly show the organic kerogen nanopores (Fig. 24c) [140]. NMR spectra of oil/water/air-shale rock crushed to powder and suspended in $\mathrm{D}_{2} \mathrm{O}$ (Fig. 25a) showed an intense water peak at $4.7 \mathrm{ppm}$ as well as broad signals at $1.1 \mathrm{ppm}$ and $6.0 \mathrm{ppm}$ typical of the aliphatic and aromatic oil proton ranges respectively, revealing that the untreated rock contained enough residual water and oil [22]. Quantitative ESR spectroscopy identifies the typical six-lines of hyperfine structure of paramagnetic $\mathrm{Mn}^{2+}$ ions $(\mathrm{S}=5 / 2)$ and determined a density of $\eta_{S}=4.50 \times 10^{19} \mathrm{Mn}^{2+}$ ions per gram of shale-oil sample in a single environment (Fig. 25a) [22]. Moreover, no ESR peaks associated to the radicals present in bitumen were observed. It has been possible to estimate surface density of paramagnetic relaxation sinks $\sigma_{s}=2.39 \times 10^{13} \mathrm{Mn}^{2+} / \mathrm{cm}^{2}$ that act to relax the liquid proton species moving in proximity of the pore surface by assuming a homogeneous distribution of paramagnetic ions in the sample. The distribution of $T_{2}$ values for petroleum liquids embedded in these rocks was measured using a Carr-PurcellMeiboom-Gill (CPMG) sequence (using a Bruker minispec at 20 $\mathrm{MHz}$ and $35^{\circ} \mathrm{C}$ ) and was found to be bimodal (Fig. 25b), extending over two orders of magnitude and with peaks centered at $T_{2}=0.55$ ms and $T_{2}=15 \mathrm{~ms}$, representing $72 \%$ and $28 \%$ of the ${ }^{1} \mathrm{H}$ population, respectively [22]. Similarly, a bimodal distribution of $T_{1}$ values was measured at $21.8 \mathrm{MHz}$ (Fig. 25b). However, these 1-D NMR techniques were not capable of separating the contributions of water and oil protons to the observed bimodal distributions.

Fig. 26a shows the 2-D NMR $\mathrm{T}_{1}-\mathrm{T}_{2}$ correlation experiments that were measured at $2.5 \mathrm{MHz}$ on untreated oil/water/air shale samples by Korb et al. [22] using a pulse sequence introduced by Mutina and Hürlimann [128] where the $T_{1}$ editing was achieved by an inversion-recovery sequence immediately followed by a long train of refocusing pulses in a $T_{2}$-CPMG sequence. The $2 \mathrm{D}$ correlation map (Fig. 26a) was finally obtained by a homemade 2D inverse Laplace program. As pointed out in Refs. [29,30], the observation of
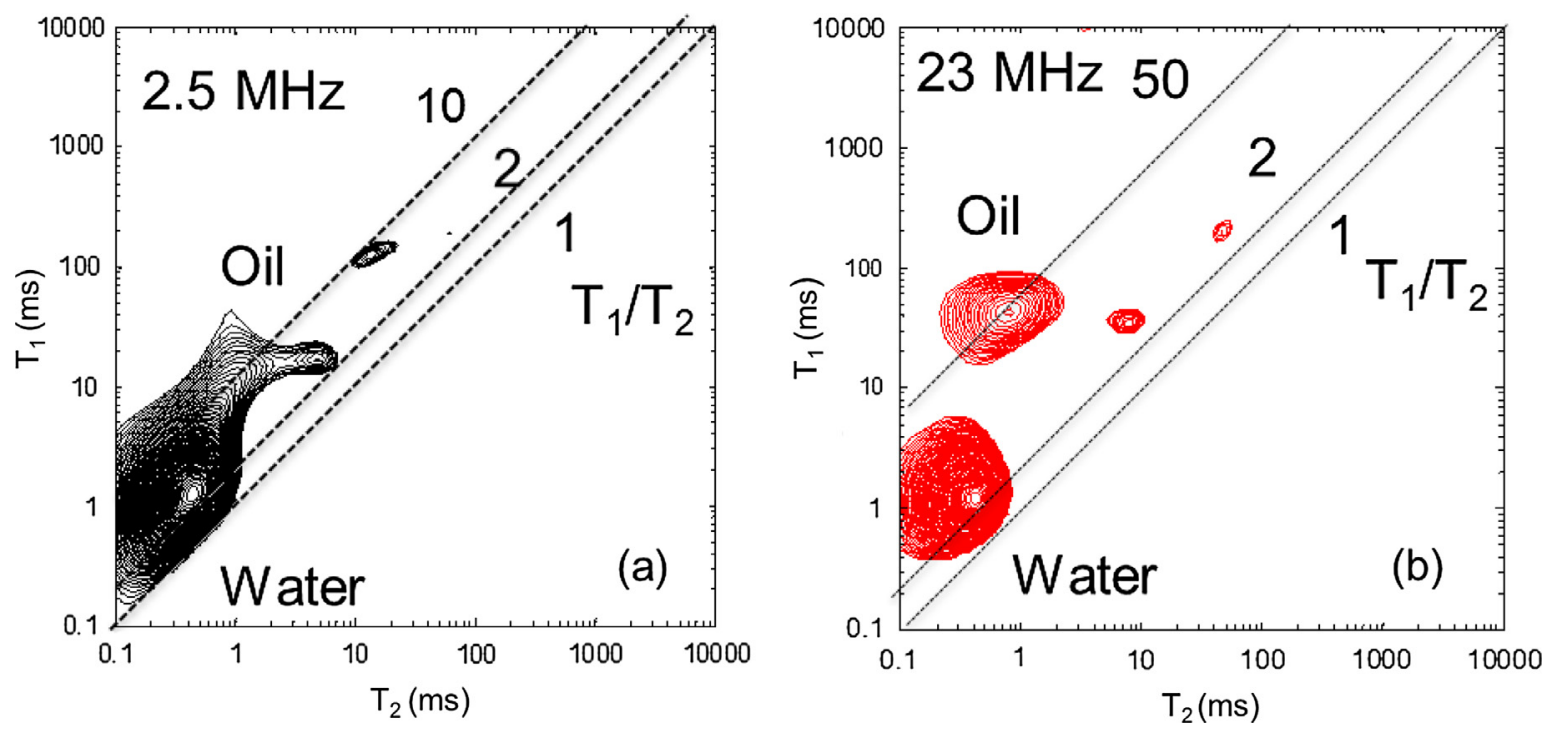

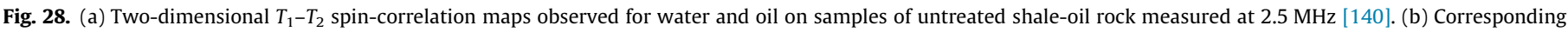
$T_{1}-T_{2}$ data measured at $23 \mathrm{MHz}$. The dashed lines indicate the values of $T_{1} / T_{2}$ ratios. The data are taken from Fig. 3 of Ref. [140]. 

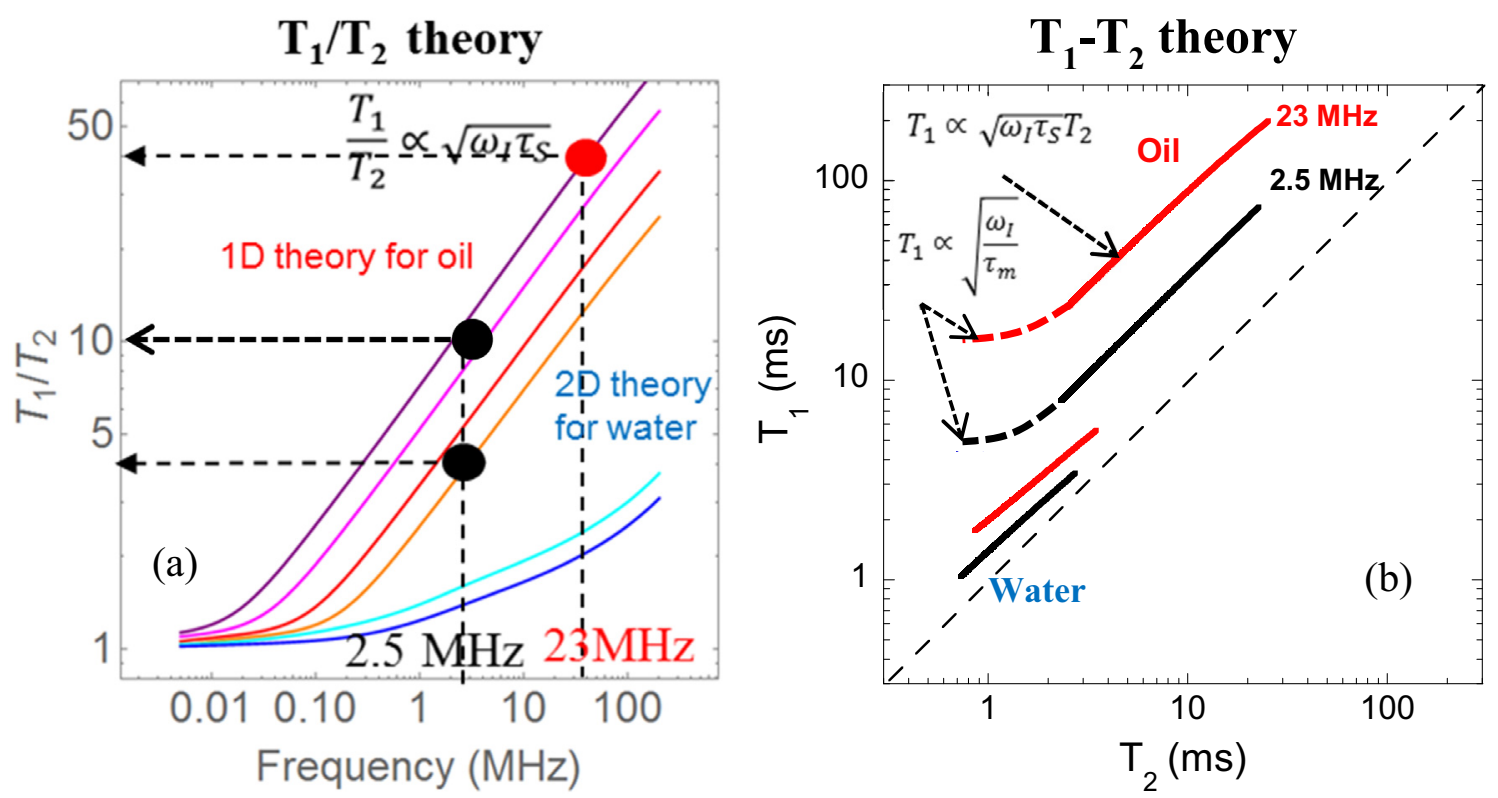

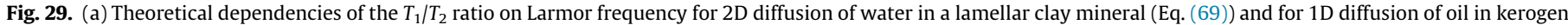

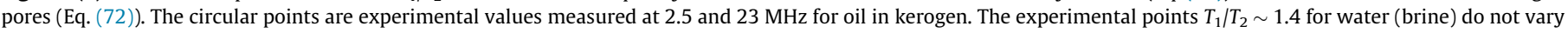

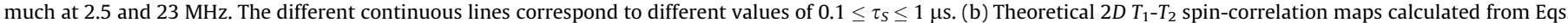

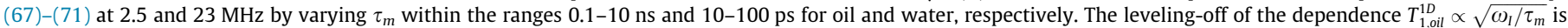

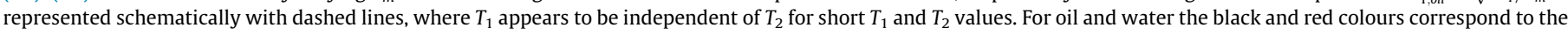
theoretical calculation of $T_{1}-T_{2}$ made at 2.5 and $23 \mathrm{MHz}$, respectively. The data are taken from Fig. 4 of Ref. [140].

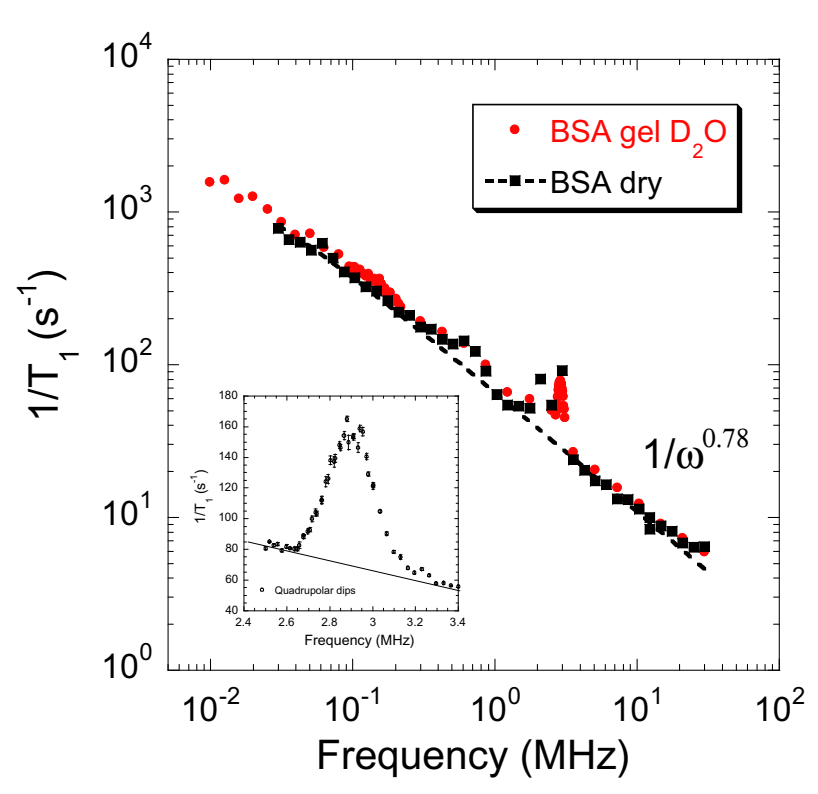

(a)

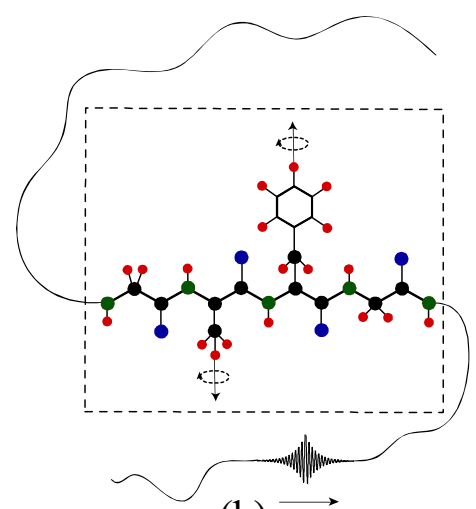

(b)

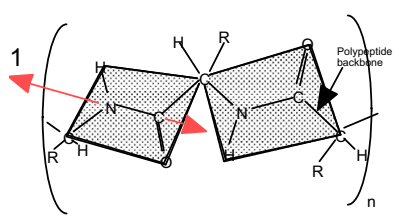

(c)

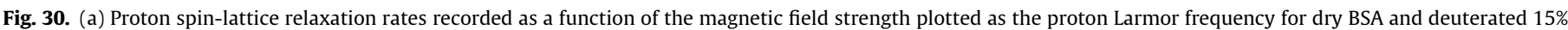

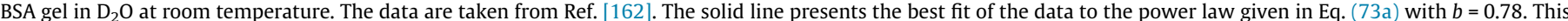

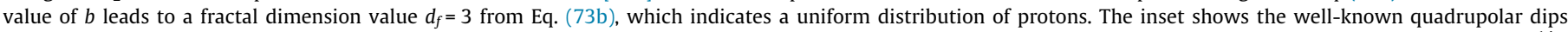

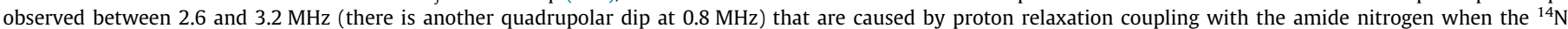

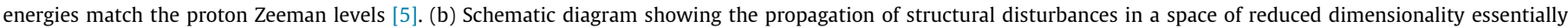
along the stiff connections of the polypeptide chain. (c) Schematic diagram showing the highest vibrational Amide (II) mode described in Section 9.1.

off-diagonal peaks in $T_{1}-T_{2}$ or $T_{2}-T_{2}$ correlation plots can be attributed to proton-water exchange between connected micropores. Other experiments have confirmed this analysis [141]. The absence of cross peaks in Fig. 26a thus proves the absence of proton exchange between oil and water populations at least on the time scale of the experiment. Moreover, no significant bitumen peaks were observed on these $2 \mathrm{D}$ spin-correlation map at short $T_{2}$ and long $T_{1}$, contrary to the situation reported by Fleury and 
Romero-Sarmiento for samples from the Barnett shale reservoir [142]. On the contrary, it has been proposed that 2D results from $T_{1}-T_{2}$ experiments on heptane-saturated kerogen isolates may be affected by bitumen [143]. The $2 D$ NMR $T_{1}-T_{2}$ correlation experiments have been repeated on a gas-shale sample where only water is present (Fig. 26b) [22]. This simplification of the 2D correlation spectrum allows identifying the highly confined water population at low values of $T_{1}, T_{2}$. The other elongated peak in Fig. 26a can thus be assigned to a confined oil population that surprisingly exhibits a high $T_{1} / T_{2}$ ratio varying from 10 to 5 . This could be due to the presence of bitumen, but this seems unlikely since the two studied shale samples come from a field producing light oil. The explanation of such a high $T_{1} / T_{2}$ ratio for the oil response should therefore be sought from a different NMR experiment.

Fig. 27a shows NMRD data obtained by Korb et al. from samples of oil/water/air rocks at room temperature $[22,140]$. The inset of Fig. 27a shows some examples at different frequencies of the bimodal distributions of $T_{1}$ illustrating that the oil and water contributions are well resolved, making possible a detailed analysis that yielded the two strikingly different NMRD profiles observed (Fig. 27a) [22]. It was thus possible to assign the water and oil NMRD profiles to the quasi-logarithmic and inverse-squared root NMRD profiles, respectively (Fig. 27a).

8.4.2. Theoretical model for interpreting the logarithmic behaviour of NMRD data of brine confined in shale rocks

One assumes the general biphasic fast exchange model where the exchange time between the proton-water transiently belonging to the surface and the bulk in pores is shorter than their respective relaxation times. When considering the lamellar clay mineral modelled as a 2D system (Fig. 27b) studied by Korb et al. $[22,140]$ the main contribution of the proton relaxation was shown to come from 2D translational proton-water $(I)$ diffusion in the proximity of fixed paramagnetic relaxing sinks $\left(\mathrm{Mn}^{2+}\right)$ of spins $S$ $=5 / 2$ that modulates their relative dipole-dipole interaction. The numerous 2D molecular re-encounters $\left({ }^{1} \mathrm{H}-\mathrm{Mn}^{2+}\right)$ are responsible for the bi-logarithmic frequency dependence of $R_{1}$ shown as a blue line in Fig. 27a (see Section 6.1) which leads to the following equation:

$$
\begin{aligned}
& R_{1, \text { water }}\left(\omega_{I}\right)=R_{1, \text { bulk }}+\left(\frac{\mu_{0}}{4 \pi}\right)^{2}\left[\pi /\left(30 \delta_{\text {water }}^{3}\right)\right] \\
& \quad \times \sigma_{S} \rho_{\text {water }} S_{p, \text { NMR }}\left(\gamma_{I} \gamma_{S} \hbar\right)^{2} S(S+1) \tau_{m} \\
& \left.\quad \times\left[3 \ln \left(\frac{1+\omega_{I}^{2} \tau_{m}^{2}}{\left(\tau_{m} / \tau_{s}\right)^{2}+\omega_{I}^{2} \tau_{m}^{2}}\right)+7 \ln \frac{1+\omega_{S}^{2} \tau_{m}^{2}}{\left(\tau_{m} / \tau_{s}\right)^{2}+\omega_{S}^{2} \tau_{m}^{2}}\right)\right]
\end{aligned}
$$

The model for which this equation was derived considers a thin surface layer $\lambda$ of the order of a molecular size, $\delta_{\text {water }} \sim 0.3 \mathrm{~nm}$, corresponding to the average distance of minimal approach between proton-water and $\mathrm{Mn}^{2+}$ ions. As usual, the translational correlation time $\tau_{m}$ is associated with individual water molecular jumps at pore surfaces. The surface residence time, $\tau_{s}\left(\gg \tau_{m}\right)$, which is limited by molecular desorption from the surface layer $\lambda$, controls how long the proton species $I$ and $S$ stay correlated at pore surfaces. The ratio $\tau_{s} / \tau_{m}$ thus represents the dynamical surface affinity or NMR wettability [27]. The best fits obtained with Eq. (67) are displayed as blue continuous lines in Fig. 27a, based on an NMR specific surface area of $S_{p, N M R}=47 \mathrm{~m}^{2} / \mathrm{g}, \tau_{m}=12.0 \mathrm{ps}$ and the frequency independent bulk value of $R_{1, \text { bulk }}^{\text {water }} \approx 30 \mathrm{~s}^{-1}$. In order to take into account the rather dispersed data at low frequency, calculated NMRD profiles were obtained by varying the activation energy $5 \leq E_{S} \leq 7.5 \mathrm{kcal} / \mathrm{mol}$ assumed for the residence times $\tau_{S}=\tau_{S 0} \exp \left(E_{S} / R T\right)$ at pore surfaces, and are shown in Fig. 27a. At high frequency $\left(\omega_{I}>0.3 \mathrm{MHz}\right)$ all the individual fitting curves merge to just one characterized by a single time of residence

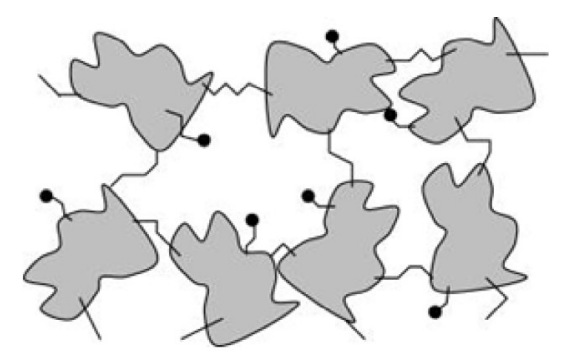

(a)

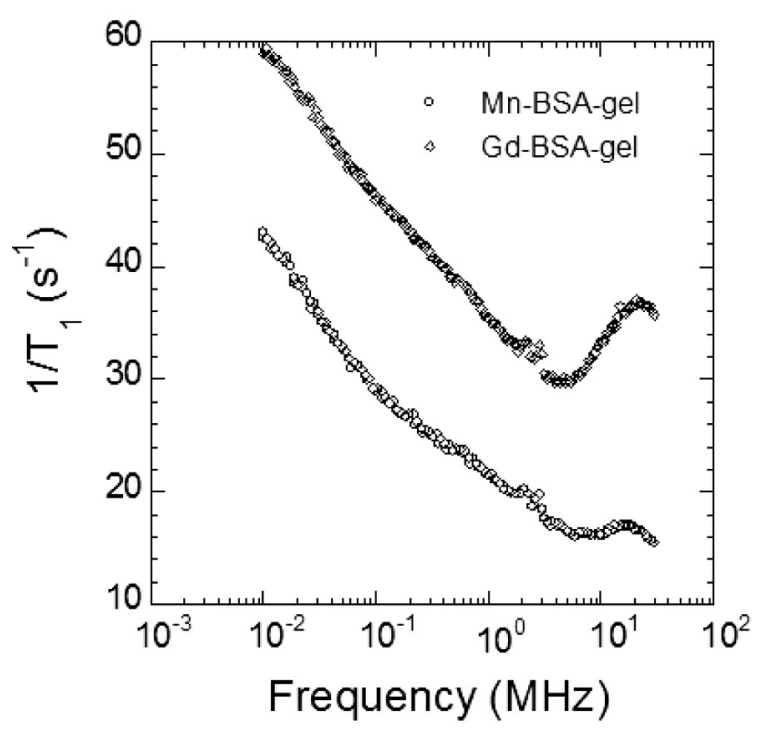

(b)

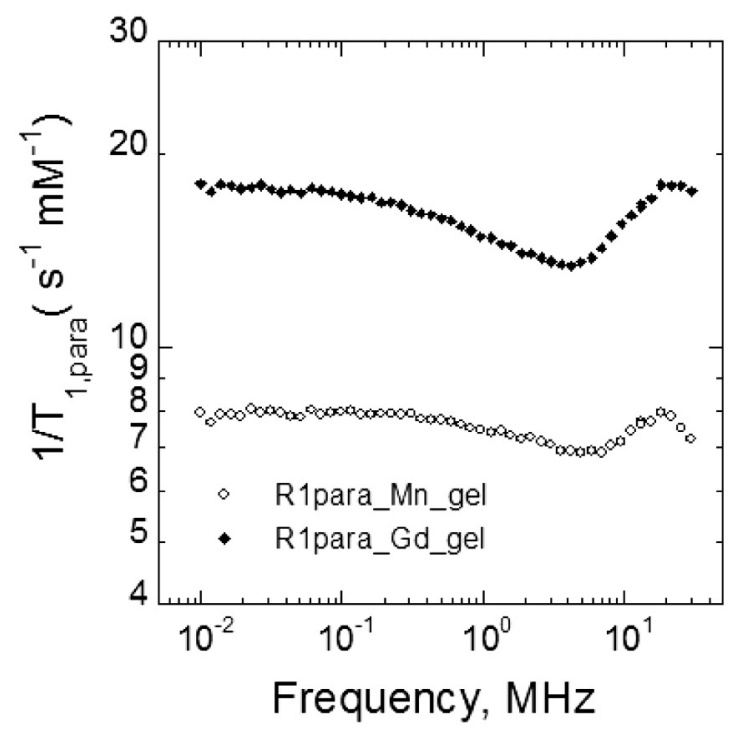

(c)

Fig. 31. (a) Schematic representation of a cross-linked protein gel showing a metal chelate complex attached by an amide linker at lysine side chains. (b) The dependence on Larmor frequency of the water-proton spin-lattice relaxation rate constant at $25{ }^{\circ} \mathrm{C}$ for $15 \%$ bovine serum albumin (BSA) cross-linked with glutaraldehyde (at ice temperature) and covalently labeled at lysine with three equivalents of DTPA anhydride. These protein gels contained 1.0 equivalent of Mn (II) ion or $\mathrm{Gd}(\mathrm{III})$ ion. (c) The magnetic field dependence of the paramagnetic contribution to the water-proton spin-lattice relaxation rate constant for 15\% BSA gels at $25{ }^{\circ} \mathrm{C}$ containing 1.0 equivalent of $\mathrm{Mn}$ (II) ion or $\mathrm{Gd}$ (III) ion. In (c), the relaxation rate constant is normalized to a relaxivity for $1 \mathrm{mM}$ concentration of metal ions. The data are taken from Ref. [164]. 

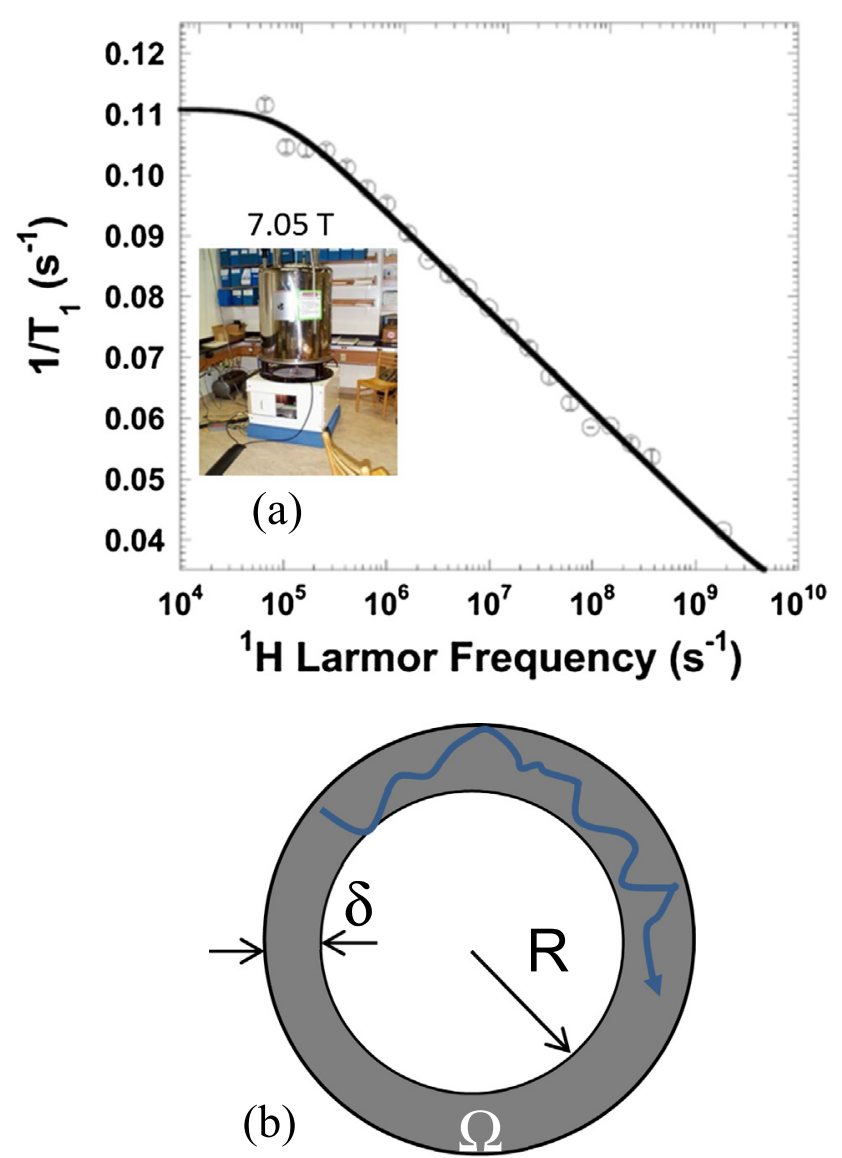

Fig. 32. (a) The residual water-proton-spin-lattice-relaxation-rate-constant as a function of proton Larmor frequency at ambient laboratory temperature for a 40 $\mathrm{mM}$ suspension of POPC in $\mathrm{D}_{2} \mathrm{O}$ (data from Ref. [35]). The inset shows the shuttle spectrometer made by R.G. Bryant [8] that was needed for collecting the NMRD data. The continuous line represents the best fit obtained using Eq. (82) according to the restricted diffusion of water represented schematically in (b).

$\tau_{s}=0.6 \mu \mathrm{s}\left(\tau_{s} \gg \tau_{m}\right)$, consistent with expectations for a water-wet situation (Fig. 27a). An estimate of the translational diffusion coefficient of water at the mineral clay-like surface may thus be made as $D_{\text {surf }}=\delta_{\text {water }}^{2} /\left(4 \tau_{m}\right)=1.88 \times 10^{-5} \mathrm{~cm}^{2} / \mathrm{s}$, which is slightly lower than expected for bulk water at $25^{\circ} \mathrm{C}$.

A treatment similar to that described above leads to the following transverse relaxation rate:

$$
\begin{aligned}
& R_{2, \text { water }}\left(\omega_{I}\right)=R_{2, \text { bulk }}+\left(\frac{\mu_{0}}{4 \pi}\right)^{2}\left[2 \pi /\left(30 \delta_{\text {water }}^{3}\right)\right] \sigma_{S} \rho_{\text {water }} S_{p, N M R}\left(\gamma_{I} \gamma_{s} \hbar\right)^{2} S(S+1) \tau_{m} \\
& \times\left[2 \operatorname{Ln}\left(\frac{\tau_{S}}{\tau_{m}}\right)+\frac{3}{4} \ln \left(\frac{1+\omega_{I}^{2} \tau_{m}^{2}}{\left(\tau_{m} / \tau_{s}\right)^{2}+\omega_{I}^{2} \tau_{m}^{2}}\right)+\frac{13}{4} \ln \left(\frac{1+\omega_{S}^{2} \tau_{m}^{2}}{\left(\tau_{m} / \tau_{s}\right)^{2}+\omega_{S}^{2} \tau_{m}^{2}}\right)\right] .
\end{aligned}
$$

Combining Eqs. (67) and (68) leads to the following theoretical expression for the ratio $T_{1, \text { water }} / T_{2 \text {,water }}$ for two-dimensional diffusion [140]:

$$
\frac{T_{1, \text { water }}^{2 D}}{T_{2, \text { water }}^{2 D}}=\frac{\left[\ln \left(\frac{\tau_{s}^{2}}{\tau_{m}^{2}}\right)+\frac{3}{4} \ln \left(\frac{1+\omega_{I}^{2} \tau_{m}^{2}}{\left(\tau_{m} / \tau_{s}\right)^{2}+\omega_{I}^{2} \tau_{m}^{2}}\right)+\frac{13}{4} \ln \left(\frac{1+\omega_{S}^{2} \tau_{m}^{2}}{\left(\tau_{m} / \tau_{s}\right)^{2}+\omega_{S}^{2} \tau_{m}^{2}}\right)\right]}{\left[3 \ln \left(\frac{1+\omega_{I}^{2} \tau_{m}^{2}}{\left(\tau_{m} / \tau_{s}\right)^{2}+\omega_{I}^{2} \tau_{m}^{2}}\right)+7 \ln \left(\frac{1+\omega_{S}^{2} \tau_{m}^{2}}{\left(\tau_{m} / \tau_{s}\right)^{2}+\omega_{S}^{2} \tau_{m}^{2}}\right)\right]}
$$

This gives an almost constant value for $T_{1 \text {,water }} / T_{2 \text {,water }} \approx 1.36$ for water confined in $2 \mathrm{D}$ mineral pores when $\tau_{s}$ varies between 8 and $0.6 \mu$ s at $2.5 \mathrm{MHz}$, exactly as observed by Nicot et al. in another $T_{1}-T_{2}$ experiment realized on a pure water-shale rock [144].
8.4.3. Theoretical model for interpreting the power-law behaviour of NMRD data for oil

Based on a previous proton NMRD data by Dalas et al. [145] on the 1D pores of ettringite in cement pastes, the inverse squaredroot behaviour with a leveling-off at low frequency shown as a red continuous line in Fig. 27a strongly indicates a relaxation process induced by a quasi-1D-translational diffusion of proton-oil species in proximity of paramagnetic $\mathrm{Mn}^{2+}$ ions at surface of kerogen micropores (Fig. 27c). Here again, the dominant feature of this relaxation process is the time dependence of the probability of reencounters between moving protons $I$ and fixed paramagnetic spins $S$ evolving at long times as $P(\tau) \propto \exp \left(-\tau / \tau_{S}\right) / \sqrt{ }\left(\tau / \tau_{m}\right)$. This gives the behaviour $R_{1} \propto 1 / \sqrt{ } \omega_{I}$ shown in Fig. 27a at high frequency. The 3D microstructure of kerogen has been recently characterized with SEM and STEM imaging, including use of the FIB technique for removing very thin layers [146]. The 3D microstructure is sponge-like with a high surface area and a huge number of quasi-1D connected kerogen pores of sizes in the range $2.5-7 \mathrm{~nm}$ [146]. The measured fractal pore-size distribution gives a number of pores: $N(R) \propto\left(R / R_{\max }\right)^{-D f}$ of size $R$ in the range $\left(R_{\min } \sim 2.5 \mathrm{~nm}-\right.$ $\left.R_{\max } \sim 630 \mathrm{~nm}\right)$ with the fractal dimension $D_{f} \sim 2.3$ [146]. The divergence of such a distribution for small nanopores means that the largest number of pores $N(R)$ is around $R \sim 2.5-3 \mathrm{~nm}$. The following relation [22] for the longitudinal rate induced by translational diffusion of a liquid confined in 1-D cylindrical nanopores (Fig. 27c) explains all the features shown in Fig. 27a:

$$
\begin{aligned}
R_{1, \text { oil }}\left(\omega_{I}\right)= & R_{1, \text { bulk }}^{\text {oil }}+\left(\frac{\mu_{0}}{4 \pi}\right)^{2} \sqrt{2} /\left(15 \pi R \delta_{1 D, \text { oil }}^{2}\right) \\
& \times \sigma_{s} \rho_{\text {oil }} S_{p, N M R}\left(\gamma_{I} \gamma_{s} \hbar\right)^{2} S(S+1) \sqrt{\tau_{m} \tau_{s}} \\
\times & {\left[\frac{3 \sqrt{1+\sqrt{1+\omega_{I}^{2} \tau_{s}^{2}}}}{\sqrt{1+\omega_{I}^{2} \tau_{s}^{2}}}+\frac{7 \sqrt{1+\sqrt{1+\omega_{S}^{2} \tau_{s}^{2}}}}{\sqrt{1+\omega_{S}^{2} \tau_{s}^{2}}}\right] . }
\end{aligned}
$$

where $R_{1, b u l k}^{\text {oil }}=1 \mathrm{~s}^{-1}$ does not have a frequency dependence in the range studied [43], the oil density is $\rho_{\text {oil }}=0.85 \mathrm{~g} / \mathrm{cm}^{3}$ and $R \sim 3.0$ $\mathrm{nm}$ is the pore radius of the largest population of pores following the fractal distribution described above. $\delta_{1 \text {, oil }} \sim \delta_{\text {oil }} / 2$ is the distance of minimal approach between $I$ and $S$ spins where $\delta_{\text {oil }}$ is the average size of hydrocarbon molecule (octane). This distance is taken to be half of the molecular size because the saturated oil has no hydrogen-bonding interactions with the paramagnetic source of relaxation. The continuous red curves of Fig. 27a for oil have been fitted with Eq. (70), with $S_{p, N M R}=30 \mathrm{~m}^{2} / \mathrm{g}$ and $\tau_{m}=4.1$ ns for different values of the activation energy $6.6 \leq E_{S} \leq 7.4 \mathrm{kcal} /$ mol associated to the activated time of residence $\tau_{S}=\tau_{S 0} \exp \left(E_{S} / R T\right)$ for considering the rather dispersed data at low frequency. The distribution of $E_{S}$ only affects the NMRD profile $1 / T_{1}\left(\omega_{I}\right)$ below $0.3 \mathrm{MHz}$. In the high frequency range where all the fits merge to a single theoretical profile $1 / T_{1}\left(\omega_{I}\right) \propto 1 / \sqrt{ } \omega_{I}$, a single surface residence time $\tau_{s} \sim 0.78 \mu \mathrm{s}$ was found. An estimation of the translational diffusion coefficient of oil at the surface of kerogen pores thus gives $D_{\text {surf }}=\delta_{\text {oil }}{ }^{2} /\left(4 \tau_{m}\right)=2.58 \times 10^{-7} \mathrm{~cm}^{2} / \mathrm{s}$. The dynamical surface affinity index $A=\tau_{s} / \tau_{m} \sim 195$ qualifying the average number of molecular steps on the pore surface gives a measure of local NMR wettability [27], and such a value is typical of an oil-wet situation in kerogen pores. The very small value of $D_{\text {surf }}$ compared to the bulk octane is due to the oil-wet-condition favouring the dynamical surface affinity of the highly confined oil in kerogen nanopores. From these $\tau_{s}$ and $D_{\text {surf }}$ values, it is possible to obtain the average pore size $\ell_{p}$ by the diffusion relation: $\ell_{p}=\sqrt{2 D_{\text {surf }} \tau_{S}}=6.4 \mathrm{~nm}$ (Fig. 27c). This value just gives an estimation of the pore length associated to the largest population of nanopores. 
A treatment similar to that described above leads to the following transverse relaxation rate for oil:

$$
\begin{aligned}
R_{2, \text { oil }}\left(\omega_{I}\right)= & R_{2, \text { bulk }}^{\text {oil }}+\left(\frac{\mu_{0}}{4 \pi}\right)^{2} 2 \sqrt{2} /\left(15 \sqrt{2} \pi R \delta_{1 D, o i l}^{2}\right) \\
& \times \sigma_{s} \rho_{\text {oil }} S_{p, N M R}\left(\gamma_{I} \gamma_{s} \hbar\right)^{2} S(S+1) \sqrt{\tau_{m} \tau_{s}} \\
\times & {\left[1+\frac{3 \sqrt{2}}{8} \frac{\sqrt{1+\sqrt{1+\omega_{I}^{2} \tau_{s}^{2}}}}{\sqrt{1+\omega_{I}^{2} \tau_{s}^{2}}}+\frac{13 \sqrt{2}}{8} \frac{\sqrt{1+\sqrt{1+\omega_{s}^{2} \tau_{s}^{2}}}}{\sqrt{1+\omega_{s}^{2} \tau_{s}^{2}}}\right] . }
\end{aligned}
$$

8.4.4. Theoretical model for interpreting the high values of the ratio $T_{1} / T_{2}$ for the oil phase

Combining Eqs. (70) and (71) and neglecting the bulk to the surface contributions, the following ratio $T_{1, \text { oil }}^{1 D} / T_{2, \text { oil }}^{1 D}$ for oil confined in 1-D micropores of kerogen is obtained, which depends only on the time of residence $\tau_{s}$ at the pore surface and is independent of all the other NMR parameters [140]: functional control [147-151]. The very complex dynamic behaviour of a folded polymeric structure may span many decades in frequency or time $[152,153]$, although for technical reasons associated with the techniques employed most recent NMR discussions of protein dynamics focus on timescales of nanoseconds and shorter. However, these time periods are far shorter than those mostly associated with biological functions. Other techniques are thus necessary to report on protein dynamics on much longer timescales, and this provides the motivation for using proton NMRD to probe protein dynamics on the timescale range of tens of microseconds to nanoseconds $[154,155]$. FFC NMRD can explore the low frequency range from $10 \mathrm{kHz}$ to $40 \mathrm{MHz}$, well below that usually addressed by high-resolution NMR methods [151] and complementary to, but more extensive than, transverse relaxation dispersion measurements [156]. The NMRD technique can thus report on protein dynamics over a range from fast, highly localized motions to slow and delocalized collective motions that may involve the whole protein. For probing intramolecular and slow protein dynamics, measurements of rotationally immobilized proteins have been a particular focus. These systems are excellent

$\frac{T_{1, \text { oil }}^{1 D}}{T_{2, \text { oil }}^{1 D}} \approx 2 \sqrt{2} \frac{1+(3 \sqrt{2} / 8) \sqrt{1+\sqrt{1+\omega_{I}^{2} \tau_{s}^{2}}} / \sqrt{1+\omega_{I}^{2} \tau_{s}^{2}}+(13 \sqrt{2} / 8) \sqrt{1+\sqrt{1+\omega_{s}^{2} \tau_{s}^{2}}} / \sqrt{1+\omega_{s}^{2} \tau_{s}^{2}}}{3 \sqrt{1+\sqrt{1+\omega_{I}^{2} \tau_{s}^{2}}} / \sqrt{1+\omega_{I}^{2} \tau_{s}^{2}}+7 \sqrt{1+\sqrt{1+\omega_{S}^{2} \tau_{s}^{2}}} / \sqrt{1+\omega_{S}^{2} \tau_{s}^{2}}}$.

The confined oil population gives rise to elongated peaks observed in Fig. 28a and b for an as received sample, which exhibit a surprisingly high $T_{1} / T_{2}$ ratio of around 10 at $2.5 \mathrm{MHz}$ (Fig. 28a) and of around $40-50$ at $23 \mathrm{MHz}$ (Fig. 28b). Fig. 29a shows the calculated frequency variations of $T_{1, \text { oil }}^{1 D} / T_{2, \text { oil }}^{1 D}$ calculated using Eq. (72) for all the frequencies. This ratio behaves as $T_{1, \text { oil }}^{1 D} / T_{2, \text { oil }}^{1 D} \rightarrow 1$ when $\omega_{\mathrm{I}} \rightarrow 0$ and as $T_{1, \text { oil }}^{1 D} / T_{2, \text { oil }}^{1 D} \propto \sqrt{\omega_{\mathrm{I}} \tau_{S}}$ at high frequency. The large value observed for $T_{1} / T_{2}$ (Fig. 29a) as well as the striking frequency evolution of the central peak of the $T_{1}-T_{2}$ correlation spectrum (Fig. 29b) can thus be explained by the low dimensionality of diffusion for the oil phase at the very large kerogen pore surface.

8.4.5. Experiments vs theory of $T_{1}-T_{2}$ at different frequencies for oil and water presenting different diffusion dimensionalities

Fig. 29a and b shows calculated data for $T_{1} / T_{2}$ and $T_{1}-T_{2}$ for oil and brine embedded in shale oils rocks at 2.5 and $23 \mathrm{MHz}$. The theoretical 2D spin-correlation spectra calculated at these frequencies with Eqs. (67), (70) for oil and water are shown in Fig. 29b, and reproduce quite well the main relaxation features observed in the experimental data (Fig. 28a and b). Note that, unlike the water peak, the oil peak moves substantially with increasing frequency; this is in excellent agreement with the theory. This result is of particular importance because it shows that measuring $T_{1}-T_{2}$ at different frequencies gives confidence in characterizing the type of fluid present in confinement.

\section{Dynamics in macromolecules and proteins}

\subsection{Noise and functional protein dynamics}

It is well known that structural fluctuations in proteins are critically important in providing access to functional conformations that are directly coupled to chemical reactivity, transport, or models for spin relaxation in biological tissues and the theoretical description that has been developed accounts well for interpreting the NMRD profiles in tissues. NMRD experiments and theories provide important insights for how intramolecular protein dynamics may affect molecular function. Rotational immobilization means that strong dipolar couplings between all the protons produce a broad homogeneous NMR spectrum approximately $25 \mathrm{kHz}$ wide. This favours an efficient and rapid spin-spin communication within the immobilized protein-proton spin system. In conse-

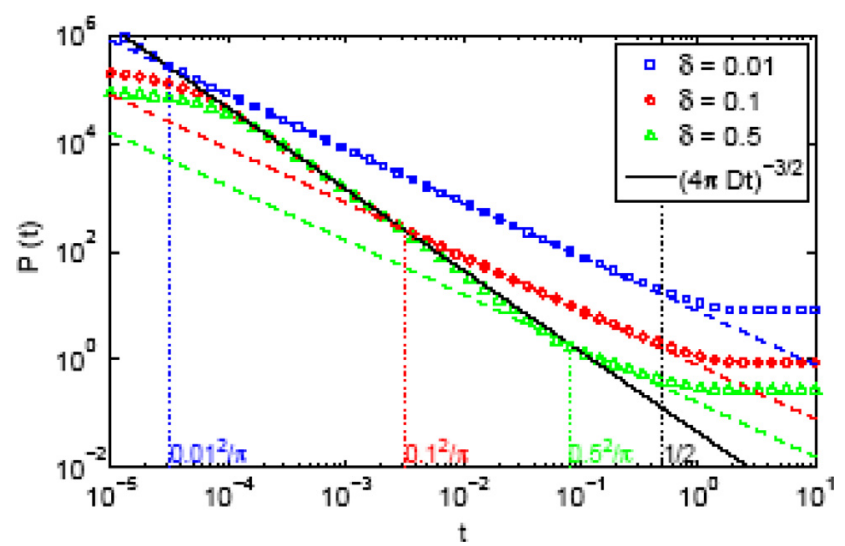

Fig. 33. Calculated probability density $P(t)$ for a random walk to return to the starting boundary point on the inner boundary of a spherical layer of inner radius $R$, outer radius $R+\delta=1$ and thickness $\delta=0.01$ (squares), $\delta=0.1$ (circles), and $\delta=0.5$ (triangles) [39]. The corresponding dashed lines show the $t^{-1}$ behaviour in the intermediate regime, while the black solid line shows the very short-time behaviour $t^{-3 / 2}$. The first three vertical dotted lines indicate the crossover times $t_{\min }=\delta^{2} / \pi$ for each spherical layer, the last one gives $t_{\max }=1 / 2($ with $D=1)$. For very short times, there is a deviation from the very short-time asymptotic behaviour (for $\delta=0.1$ and $\delta=0.5$ ) because of an insufficient number of eigenvalues used in the numerical computation. 
quence, measurements of proton spin relaxation provide a global report of protein dynamics; NMRD profiles provide direct characterization of these molecular dynamics from milliseconds to several picoseconds by changing the NMR observation window, which is linear in magnetic field strength [157]. In the lowfrequency domain, rapid internal motions such as methyl group rotation are not efficient in promoting relaxation [158]. In the absence of rapid rotational averaging, the usual approaches to spin relaxation based on a semi-classical treatment, including spectral density models, are inappropriate. The successfully developed quantum theoretical description of these experiments is based on simple assumptions that are summarized below, and which are supported in detail by experiment $[154,155]$. Basically, this quantum description rests on the fact that the stiff connections within the protein structure occur along the polypeptide chain, which makes the system behave in some respects approximately as though it were one-dimensional, rather than three-dimensional. The key point that results from such a dimensionality reduction is that structural fluctuations in the protein are far more prevalent in the frequency range usually associated with protein function than that expected from three-dimensional models. Last, anomalous relations exist between proton-spin-lattice relaxation rates and commonly encountered frequency dependences of force constants and mean-square displacements. Though NMRD measurements explore a much lower frequency range than most other techniques, these anomalous relations give additional insight into low-frequency protein dynamics. Recently, significant progress in NMR instrumentation has allowed an exploration of the spectrally resolved proton-protein dynamics over a large range of high frequencies by using a special dedicated shuttle NMR spectrometer [11]. This method, which gives dynamical information on fast segmental molecular motions, is thus complementary to the usual NMRD method, which is restricted to reporting on slow, collective molecular motions.

The proton NMRD profile $R_{p}\left(\omega_{0}\right)$ for rotationally immobilized proteins shows that there is a dramatic increase in the population of low-frequency motions from $10 \mathrm{kHz}$ to $20 \mathrm{MHz}$, which is attributed to the quasi-one-dimensional character of the protein primary structure. As a consequence, the rate (or probability) at which functionally important conformational states are thermally sampled in a protein are also dramatically increased, relative to the situation for a three-dimensional lattice structure. The relaxation rate $R_{p}\left(\omega_{0}\right)$ is found to decrease with increasing Larmor frequency according to a power law (Fig. 30a) that derives from the distribution of dynamical states, the propagation of the structural disturbances (Fig. 30b), and the spatial distribution of hydrogen atoms in the structure $[154,155]$ :

$R_{p}\left(\omega_{0}\right)=\frac{9 \pi \beta}{5} \frac{k_{B} T}{\hbar} d_{S} \omega_{\text {dip }}^{2}\left(A_{/ /}+A_{\perp}\right) \omega_{0}^{-b}$,

where the exponent $b$ is defined as:

$b=3-2 \frac{d_{S}}{d_{f}}-d_{s}$.

In Eq. (73a), $A_{/ /}=(3 / 4)\left(1+2^{-b}\right) \Omega^{b-2}, A_{\perp}=(1 / 6)\left(7 / 2+2^{-b}\right) \Omega_{\perp}^{b-2}$, $\beta$ is a numerical factor ( $\beta \sim 3$ see Ref. [155]) associated with the effective average proton dipolar coupling $\omega_{\text {dip }} / 2 \pi=11.3 \mathrm{kHz}$; and $\Omega_{/ /}$and $\Omega_{\perp}$ correspond to the frequencies of the highest vibrational modes parallel and perpendicular to the polypeptide chain, taken as the amide (I) and (II) (Fig. 30c) modes at $1560 \mathrm{~cm}^{-1}$ and 200 $\mathrm{cm}^{-1}$, respectively [159]. In Eq. (73b), $d_{S} \sim 4 / 3$ is the spectral dimension $[160,161]$ associated with the propagation of the structural disturbance (Fig. 30b), or is dependent on the number of sites sampled by a random exploration of the space characterized by a mass fractal dimension $d_{f}$ (for further details see Refs. [154,155]).
The frequency dependence of $R_{p}\left(\omega_{0}\right)$ (Eqs. (73a), (73b)) coming from the anomalous dispersion between space and frequency [161] thus allows probing of the internal protein dynamics from the fast (localized) motions to the slow cooperative (delocalized) motions. Fig. 30a shows typical proton NMRD profiles obtained by Korb and Bryant for lyophilized bovine serum albumin (BSA) and BSA trapped at $15 \%$ in $\mathrm{D}_{2} \mathrm{O}$ at room temperature $[154,155,162]$. The lyophilisation procedure is described by Lester and Bryant in Ref. [84]. The interest of trapping the BSA in gel is to quench the protein rotation allowing observing the internal protein dynamics. The inset of Fig. 30 shows the well-known quadrupolar dips observed between 2.6 and $3.2 \mathrm{MHz}$ (there is another quadrupolar dip at $0.8 \mathrm{MHz}$ ) that are caused by proton relaxation coupling with the amide nitrogen when the ${ }^{14} \mathrm{~N}$ energies match the proton Zeeman levels [5].

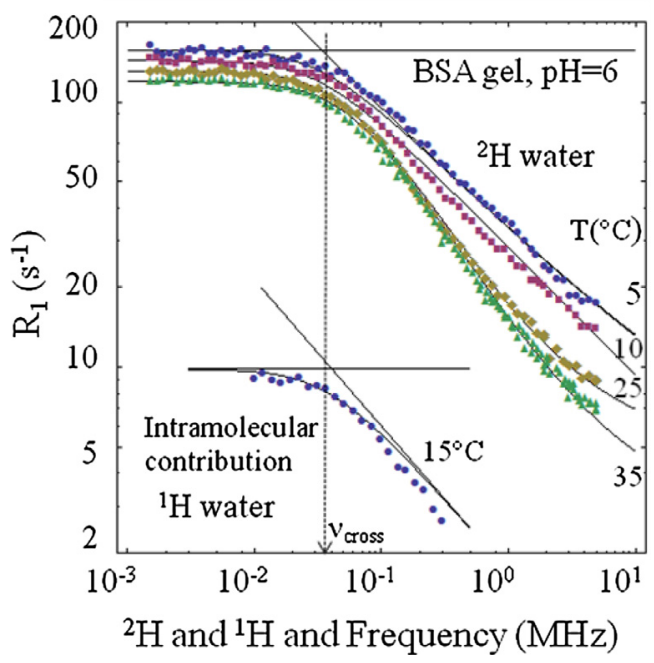

Fig. 34. Deuteron spin-lattice relaxation rates plotted against deuteron Larmor frequency for $15 \% \mathrm{BSA}$ gel in $\mathrm{D}_{2} \mathrm{O}$ at $\mathrm{pH}=6$ and at $5,10,25$ and $35^{\circ} \mathrm{C}$. The intramolecular contribution of the proton spin-lattice relaxation rate is also plotted against the proton Larmor frequency for the same BSA gel in $\mathrm{H}_{2} \mathrm{O}$ at $15^{\circ} \mathrm{C}$. The data are taken from Ref. [38]. The continuous lines represent the best fits obtained using equations given in Ref. [38] where $v_{\text {cross }} \approx 40 \mathrm{kHz}$ corresponds to the same transition occurring between the power-law frequency dependence and the plateau observed for ${ }^{1} \mathrm{H}$ and ${ }^{2} \mathrm{H}$.

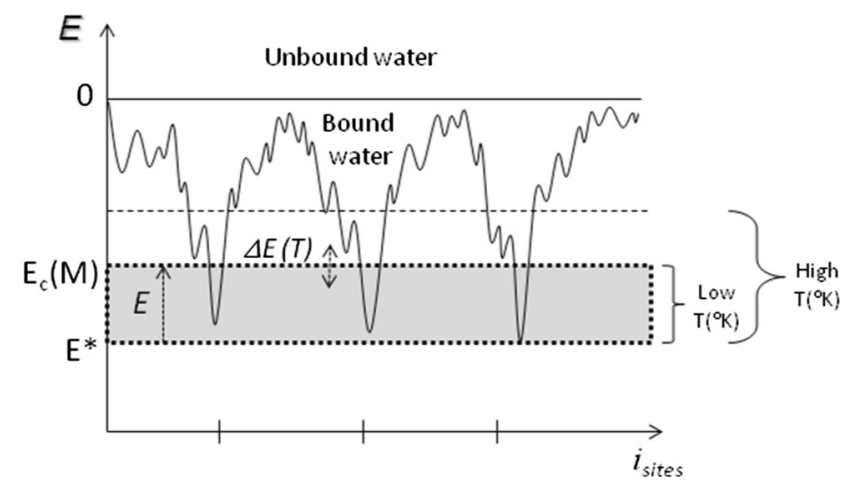

Fig. 35. Schematic diagram intended to represent the rugged energy landscape of the very large number of potential binding sites $I \in[1, \ldots, M]$ for water in the hydration layer at the surface of an immobilized protein. Here, $\Delta E(T)$ represents the temperature-dependent thermal fluctuations that allow exploration of a certain range of energy around the lowest free energy minima $E^{*}$ belonging to the shaded region when $E^{*}>-\infty$. The values of the explored extreme minima $E^{*}$ fluctuate around some negative free energy $E_{c}(M)$ that can include a domain where the single and double well situations apply. This theoretical diagram taken from Fig. 1 of Ref [38] has been used with extreme-values statistics to interpret the "swallow" temperature dependence of the NMRD of water dynamics at the protein interface (Fig. 34). 

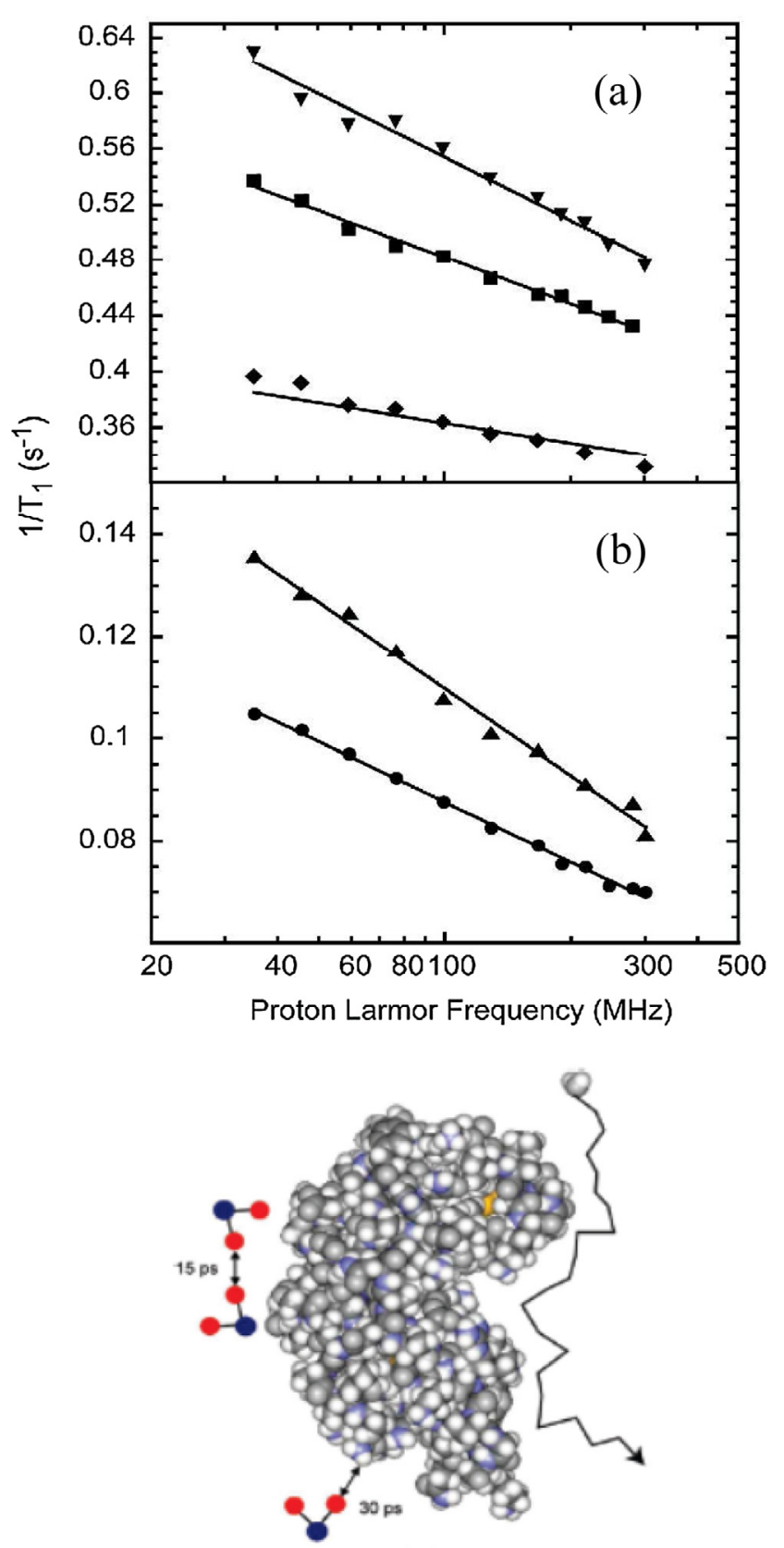

(c)

Fig. 36. (a) Proton spin-lattice relaxation rate constants as a function of proton Larmor frequency for $10 \%$ lysozyme solution in $\mathrm{H}_{2} \mathrm{O}$ (squares), $10 \%$ BSA solution in $\mathrm{H}_{2} \mathrm{O}$ (triangles pointing down), and 5.5\% ribonuclease A (diamonds) at room temperature and (b) $10 \%$ lysozyme solution in $\mathrm{D}_{2} \mathrm{O}$ (circles) and $5 \%$ ribonuclease $\mathrm{A}$ solution in $\mathrm{D}_{2} \mathrm{O}$ (triangles pointing up). The experimental data are from Ref. [39]. (c) Schematic representation of water molecule diffusion at the surface of ribonuclease A. The scale of the water molecules on the left side is enlarged to clarify the nature of self- and relative diffusion.

For both systems, the best fits obtained with Eqs. (73a) and (73b) give $R_{p}\left(\omega_{0}\right) \propto \omega_{0}^{-b}$ with $b=0.78$. The importance of local dimensionality was implied in the previous work of Kimmich et al. [163], who found that proton NMRD profiles of polypeptide chains (polyglycine), that contain no side chains, and those of proteins, which of course do have side chains, each show the same power-law exponent $b=0.78$.

The theoretical foundation for the spin-relaxation process, loosely characterized as a direct spin-phonon coupling (Eq. (73a)), shows that structural disturbances propagate in a space of

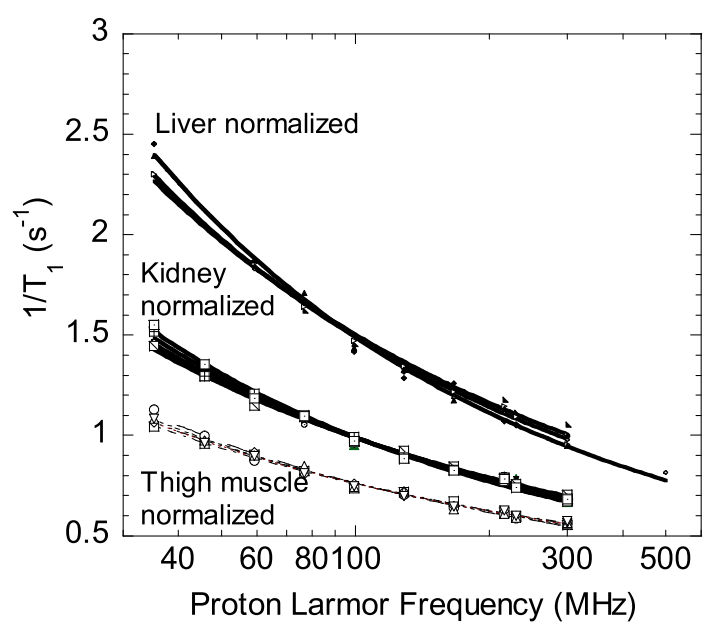

Fig. 37. ${ }^{1} \mathrm{H}$ nuclear spin-lattice relaxation rate constants as a function of proton Larmor frequency, averaged over five samples of excised rat muscle, kidney, and liver at ambient laboratory temperature $(295 \pm 2 \mathrm{~K})$. The data are taken from Ref. [40]. Solid lines are least squares fits to Eq. (83) with the parameters $A=6.4 \times 10^{4}$ in $\mathrm{s}^{-1.6}$ to respect the dimension in $\mathrm{s}^{-1}$ of the first term $\left[A \omega^{-0.6}\right]$ in Eq. (83) and $B=2.6 \times 10^{-8} \mathrm{~s}^{-2}$

reduced dimensionality, essentially along the stiff connections of the polypeptide chain (Fig. 30b). This reduced dimensionality "traps" the disturbance and changes the efficiency of energy redistribution within the protein and hence the processes that drive nuclear spin relaxation. The Larmor frequency dependence of the protein-proton spin-lattice relaxation rate constant of the protons in a protein is also related to the frequency dependence of force constants and mean-square displacements commonly observed or calculated for proteins. It has been proposed that these approaches give additional physical insight into the character of the extremely low-frequency protein dynamics [155].

\subsection{Paramagnetic relaxation of protons in rotationally immobilized proteins or crosslinked protein gels}

Numerous field-cycling relaxometry studies of protein solutions exist in the literature [36], and interesting studies for diamagnetic species have also been proposed [5]. However, proton spin-lattice relaxation induced by paramagnetic centers may be dramatically enhanced if the paramagnetic center is rotationally immobilized in the magnetic field [37,164]. In such circumstances, the details of the relaxation mechanisms acting are different from those appropriate to solutions of paramagnetic relaxation agents. Large enhancements in the proton spin-lattice relaxation rate constants associated with organic radicals are observed when the radical system is rigidly connected with a rotationally immobilized macromolecular matrix such as a dry protein or a crosslinked protein gel $[37,164]$. The paramagnetic influence on the protein-proton populations is direct and is distributed internally among the protein protons by efficient spin diffusion. In the case of a cross-linkedprotein gel, the paramagnetic effects are carried to the water spins indirectly by chemical exchange mechanisms involving both proton exchange and water molecule exchange with rare long-lived water molecule binding sites on the immobilized protein (Fig. 31a). The net increase of spin relaxation rates by organic radicals compared with metal systems at low magnetic field strengths occurs because the electron relaxation time of the radical is orders of magnitude longer than that for metal systems. This gain in relaxation efficiency in the low frequency range provides completely new opportunities for the design of relaxation-based contrast agents in magnetic resonance imaging and also provides new ways to examine intramolecular protein dynamics. 
Fig. 31a shows a schematic representation of a cross-linked protein gel [164]. The protein cross-linking agent tethers one protein molecule to another through amide linkages at lysine side chains to form a three-dimensional network, making the apparent bulk viscosity of the sample infinite; however, the local microdynamical viscosity as sensed by the water motion is essentially unaffected. The consequence is that global rotation of the protein is quenched, except for local rocking motions within the crosslinked lattice. In addition, the covalent tethers that attach the paramagnetic centers to the protein may suffer significant local motion in the gel. Fig. 31b shows the NMRD profiles of the water proton spin-lattice relaxation rate. A consequence of preventing the rotational motion of the protein in the 15\% BSA gel is that the protein proton as well as the water proton relaxation behaviours both change profoundly. The protein-proton linewidth becomes essentially equivalent to that of a solid-state sample, with a value around $25 \mathrm{kHz}$, and the relaxation dispersion profile of the protein protons changes from a Lorentzian function to one characterized by a power law. A quantitative theory has been proposed by Korb and Bryant $[154,155]$ relating the parameters of the power law to the internal dynamics of the protein involving a spin-phonon like direct relaxation process. The protein-proton relaxation dispersion is mapped onto the observed water spin relaxation dispersion by magnetic exchange processes dominated by the chemical exchange of water molecules from rare, long-lived binding sites. Relaxation rates become large at low fields and excellent signalto-noise ratios are required to extract the paramagnetic contributions to the relaxation accurately; new instrumentation has made this possible and some examples are shown in Fig. 31c for 15\% paramagnetic gels. The paramagnetic contributions to relaxation in these rotationally immobilized gels are very similar to those reported extensively for slowly rotating or protein bound paramagnetic complexes of gadolinium or manganese. The relaxation dispersion profile is characterized in both cases by a peak in the relaxation rate in the vicinity of $20 \mathrm{MHz}$, which is well known to be caused by the magnetic field dependence of the electron spin relaxation times. It is now widely realized that the SolomonBloembergen-Morgan equations [165] may be used to obtain a reasonable fit similar to those of Fig. 31c. However, while this may work at a numerical level, it is clear that such parametrization of the data is not physically realistic because of the importance of large zero-field splitting tensors for the metal centers which make the magnetic field dependence very difficult to compute accurately [166-168]. Nonetheless, it remains the case that the effects of the paramagnetic metal center on the water protons in this crosslinked protein system are very similar to those for the same metal centers covalently linked to a freely rotating paramagnetic protein in solution; there is sufficient motion of the metal center in the cross-linked gel to provide electron spin relaxation rates that are essentially similar to those for the freely rotating system. Overall, it is a reasonable approximation to understand the observed water-proton relaxation as a superposition of two essentially independent contributions: one that arises from the coupling of water with the diamagnetic protein and one that arises from the effects of the water coupling to paramagnetic centers.

\subsection{Translational dynamics of water at the phospholipid interface}

Phospholipids are critical components of living cells and measuring water and lipid dynamics at their interfaces informs on their biological functions. As a phospholipid vesicle is a very large object compared to proteins, the translational water dynamics may be biased and significantly reduced by the excluded volume created by the huge vesicle interface [35,169]. Here again NMRD is a well suited method for separating the different inter- and intramolecular relaxation contributions of water at phospholipid interfaces. Measuring the residual water-proton NMRD profile obtained from suspensions of water diffusing at the interface of 1-palmitoyl-2oleoyl-sn-glycero-3-phosphocholine (POPC) phospholipid vesicles in deuterium oxide was found by Victor, Korb and Bryant [35] to be a logarithmic function of the proton Larmor frequency at high magnetic field strengths, whereas at low magnetic field strengths it was independent of Larmor frequency (Fig. 32a). The residual proton HOD relaxation is caused by dipole-dipole couplings between the residual water protons in otherwise deuterated water and the phospholipid protons. The NMRD profile for the rare residual HOD protons in $\mathrm{D}_{2} \mathrm{O}$ has been obtained with the shuttle NMR apparatus shown in the inset of Fig. 32a. The rate constants measured in $\mathrm{D}_{2} \mathrm{O}$ are significantly smaller than those measured in $\mathrm{H}_{2} \mathrm{O}$ because both the intra and intermolecular water-water dipolar interactions have been substantially removed. The protondeuteron dipolar coupling will make a relaxation rate contribution less than $0.02 \mathrm{~s}^{-1}$. The proton relaxation rate constant becomes progressively independent of the frequency at low values and linear in the logarithm of the Larmor frequency at high values over a remarkably extended range. This is a rather surprising result for a spherical multilayer system (Fig. 32b) because this logarithmic NMRD profile is usually observed for a 2D flat surface. The interpretation of this surprising result is detailed in the following sub-section.

Table 4

Brain data renormalized with exponent 0.6 [40].

\begin{tabular}{lll}
\hline Sample & $\mathrm{A}^{*} 10^{-6}\left(\mathrm{~s}^{-1.6}\right)$ & $\tau_{d},(\mathrm{ps})^{\mathrm{a}}$ \\
\hline White matter & 0.035 & 49 \\
Grey matter & 0.040 & 9.22 \\
Caudate & 0.048 & 5.88 \\
Thalamus & 0.038 & 9.76 \\
Putamen & 0.043 & 7.58 \\
Globus palidus & 0.045 & 41.07 \\
\hline
\end{tabular}

${ }^{\text {a }}$ Fits to Eq. (83) with $B$ set to $0.6 \times 10^{9}\left(\mathrm{~s}^{-2}\right)$ and $C=0.28\left(\mathrm{~s}^{-1}\right)$.

Table 3

Tissue parameters for excised rat ${ }^{\mathrm{a}}[40]$.

\begin{tabular}{|c|c|c|c|c|c|c|}
\hline Samples & $\begin{array}{l}\text { Muscle } \\
A^{*} 10^{-6}\left(s^{-1.6}\right)\end{array}$ & $\begin{array}{l}\text { Muscle } \\
\mathrm{B}^{*} 10^{-9}\left(\mathrm{~s}^{-2}\right)\end{array}$ & $\begin{array}{l}\text { Kidney } \\
A^{*} 10^{-6}\left(s^{-1.6}\right)\end{array}$ & $\begin{array}{l}\text { Kidney } \\
\mathrm{B}^{*} 10^{-9}\left(\mathrm{~s}^{-2}\right)\end{array}$ & $\begin{array}{l}\text { Liver } \\
\mathrm{A}^{*} 10^{-6}\left(\mathrm{~s}^{-1.6}\right)\end{array}$ & $\begin{array}{l}\text { Liver } \\
\mathrm{B}^{*} 10^{-9}\left(\mathrm{~s}^{-2}\right)\end{array}$ \\
\hline 1 & 0.069 & 0.19 & 0.090 & 0.37 & 0.19 & 0.35 \\
\hline 2 & 0.054 & 0.32 & 0.087 & 0.40 & 0.14 & 0.76 \\
\hline 3 & 0.058 & 0.28 & 0.083 & 0.44 & 0.16 & 0.63 \\
\hline 4 & 0.063 & 0.24 & 0.099 & 0.30 & 0.19 & 0.31 \\
\hline 5 & 0.059 & 0.27 & 0.11 & 0.22 & 0.18 & 0.38 \\
\hline Average & 0.061 & 0.26 & 0.094 & 0.35 & 0.17 & 0.49 \\
\hline St. Dev. & 0.0057 & 0.048 & 0.011 & 0.087 & 0.022 & 0.2 \\
\hline
\end{tabular}

${ }^{\text {a }}$ Fits to Eq. (83) with $\tau_{d}=15 \mathrm{ps}$ and $\mathrm{C}=0.28\left(\mathrm{~s}^{-1}\right)$. 
9.3.1. Calculation of the dipolar correlation functions for restricted diffusion of water between two thin spherical layers

For understanding the logarithmic NMRD profile associated with the confined dynamics of water at the spherical phospholipid interface (Fig. 32a), this section describes the main steps of the calculations of the pairwise dipolar correlation functions $G_{L}^{(m)}(\tau)$ associated with translational diffusion of water in the vicinity of a spherical macromolecule (protein or vesicle) uniformly distributed in solution and schematically represented in (Fig. 32b).

The pairwise dipolar correlation function for a diffusive process inside of a spherical layer $\Omega=\left[\boldsymbol{r} \in R^{3} ; R<|\boldsymbol{r}|<R+\delta\right]$ between two reflecting boundaries (Fig. 32b) is given by the following stationary integral averaged over the diffusive conditional probability $P\left(\vec{r}_{0}, \vec{r}, \tau\right)$

$G_{L}^{(m)}(\tau)=\int_{\Omega} d \vec{r}_{0} p\left(\vec{r}_{0}\right) F_{L}^{(-m)}(0) \int_{\Omega} d \vec{r} P\left(\vec{r}_{0}, \vec{r}, \tau\right) F_{L}^{(-m)} *(\tau)$

In Eq. (74), $F_{L}^{(m)}(\tau)$ represents the dipolar interaction, $p\left(\boldsymbol{r}_{0}\right)$ is the initial water density in the layer and $P\left(\vec{r}_{0}, \vec{r}, \tau\right)$ is the conditional probability for a particular water-proton-spin $I$ to move, inside the spherical layer $\Omega$, in a given time $\tau$, from an initial position $\boldsymbol{r}_{0}$ to another position $\boldsymbol{r}$ relatively to a proton standing at the layer surface (Fig. 32b). Eq. (74) can be greatly simplified at short times (high frequency), because the water proton spins $I$ have not had time to explore the whole confining domain $\Omega$. Moreover, the rotational invariance of the problem implies that the probability of return to an initial position $\boldsymbol{r}_{0}$ within the confining domain $\Omega$ in a time $\tau, P\left(\vec{r}_{0}, \vec{r}, \tau\right)$, is independent of the angular coordinates of the starting point $\boldsymbol{r}_{0}$, i.e.

$P\left(\vec{r}_{0}, \vec{r}_{0}, \tau\right)=P(\tau)$

These considerations result in the following approximation at short times:

$G_{L}^{(m)}(\tau) \approx P(\tau) \quad(\tau \rightarrow 0)$

This means that the time dependence of $G_{L}^{(m)}(\tau)$ is equivalent to that for the probability to return to the origin. The form of $P\left(\vec{r}_{0}, \vec{r}, \tau\right)$ in the general case is governed by the solution of the diffusive equation:

$\frac{\partial}{\partial t} P\left(\vec{r}_{0}, \vec{r}, t\right)=D \Delta_{S} P\left(\vec{r}_{0}, \vec{r}, t\right)$

where $\Delta_{S}$ is the Laplacian operator at spherical surface and with the following Neumann boundary condition for $P$ on the two layer surfaces $[69,170]$ :

$\frac{\partial}{\partial n} P\left(\vec{r}_{0}, \vec{r}, t\right)=0$

where $\frac{\partial}{\partial n}$ is the normal derivative. The initial condition for $P$ has been chosen by the Dirac distribution:

$P\left(\vec{r}_{0}, \vec{r}, t=0\right)=\delta\left(\vec{r}-\vec{r}_{0}\right)$.

The solutions of Eq. (77) under these conditions have been previously established $[69,170,171]$. For a bounded diffusionconfining domain, a spectral decomposition over the eigenvalues $\lambda_{m}$ and eigenvectors $u_{m}(\boldsymbol{r})$ of the Laplacian operator $\Delta_{S}$ provides an explicit formal dependence of $P\left(\vec{r}_{0}, \vec{r}, t\right)$ as:

$P\left(\vec{r}_{0}, \vec{r}, t\right)=\sum_{m} u_{m}^{*}\left(\vec{r}_{0}\right) u_{m}(\vec{r}) e^{-D \lambda_{m} t}$

For spherical layers, the eigenfunctions of the Laplacian operator are known explicitly [69,170], which permits efficient analytical and numerical computations of the probability of return
$P(t)$. The asymptotic forms of $P(t)$ are detailed in the Appendix of Ref. [39], and behave as:

$$
\begin{aligned}
& P(t) \propto \frac{2}{(4 \pi D t)^{\frac{3}{2}}}, \quad t \ll t_{\min }=\frac{\delta^{2}}{\pi D}, \\
& P(t) \propto \frac{1}{(4 \pi D t \delta)}, \quad t_{\min } \ll t \ll t_{\max }, \\
& P(t) \propto \frac{1}{V}, \quad t \gg t_{\max }=\ell^{2} /(2 D)
\end{aligned}
$$

For very short times $\left(t \ll t_{\min }\right)$ Eq. (81a) dominates, as expected because the water diffusion cannot explore the whole domain. However, for intermediate times $\left(t_{\min } \ll t \ll t_{\max }\right)$, Eq. (81b) becomes the determinant contribution yielding $P(t) \propto 1 / t$. Finally, for very long times $t \gg t_{\max }$, the molecules explore the whole diffusion-confining domain of size $L$ many times, so that the probability of return becomes independent of the exploration time, being inversely proportional to the volume $V$ of the domain. The time dependence of such a probability of return $P(t)$ has been calculated by Grebenkov et al. [39] and is displayed in Fig. 33 for different values of the ratio $\delta / R$ (Fig. 32b). Interestingly, for a thin spherical layer $\delta / R=0.01$ one finds $P(t) \propto \frac{1}{t}$ which leads to a logarithmic spectral density $J(\omega)$. Such a result is compatible with the logarithmic frequency dependence observed for water dynamics at the interface of phospholipid vesicles (Fig. 32a) [35] as well as water diffusion observed for different protein interfaces [39].

It is also possible to obtain such logarithmic frequency dependence when the water is diffusive within spherical thin layers (Fig. 32b). These results are quite different from the magnetic field dependence of lipid-proton-relaxation dispersion [172-174]. Because the observed HOD relaxation dispersion is different from that reported by the lipid protons, the magnetic coupling or magnetization transfer between the rare residual water protons and the pure lipid protons at the interface is weak. The high field region of the relaxation dispersion has a logarithmic dependence on magnetic field strength that is characteristic of relaxation caused by diffusive exploration of a planar 2-dimensional space [71,175]. Of course the phospholipid surface is remarkably dynamic on the time scales of the relaxation times measured or the reciprocal of the Larmor frequencies utilized here, and there are no significant binding interactions to hold water at an interface. Nevertheless, the presence of the lipid occupies space that may not be simultane-

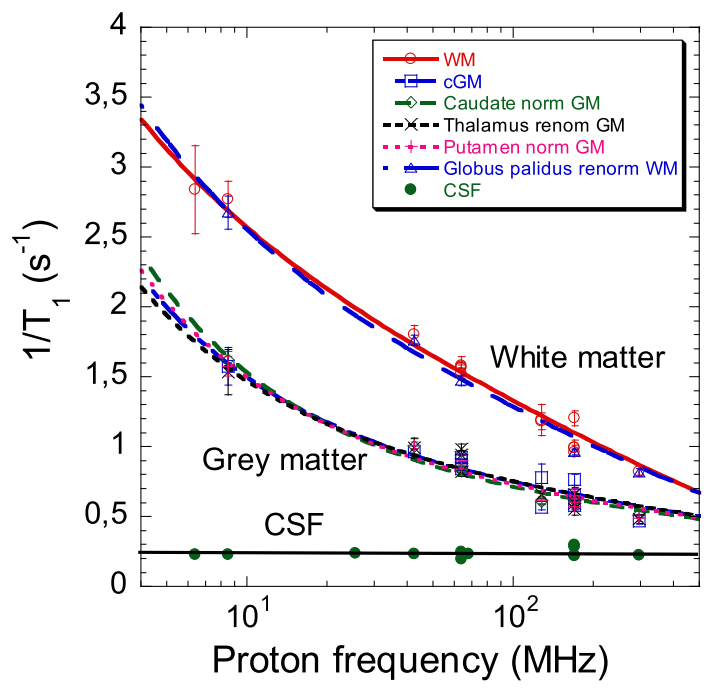

Fig. 38. Brain tissue proton relaxation rates normalized to either the grey or white matter average values. Lines are fits to Eq. (83) with the parameters summarized in Table 4. The in vivo MRI brain data are taken from Ref. [41]. 
ously occupied by water, which biases the diffusive exploration by the freely diffusing water molecules (Fig. 32b). The relaxation equation appropriate to 2-dimensional intermolecular spin dynamics in the immediate proximity (Fig. 32b) of the phospholipid interface is thus similar to Eq. (55) [25]:

$$
\frac{1}{T_{1}}=\frac{1}{T_{1}^{0}}+A \tau_{\text {diff }}\left\{\ln \left[\frac{1+\omega^{2} \tau_{\text {diff }}^{2}}{\left(\frac{\tau_{\text {diff }}}{\tau_{I}}\right)^{2}+\omega^{2} \tau_{\text {diff }}^{2}}\right]+4 \ln \left[\frac{1+4 \omega^{2} \tau_{\text {diff }}^{2}}{\left(\frac{\tau_{\text {diff }}}{\tau_{I}}\right)^{2}+4 \omega^{2} \tau_{\text {diff }}^{2}}\right]\right\}
$$

where $T_{1}{ }^{\circ}$ is the relaxation time at very high frequency, $A$ is a constant characterizing the strength of the dipolar couplings and the probability that the water proton is at the interface, $\tau_{\text {diff }}$ the correlation time for translational diffusion in the interface, $\tau_{I}$ the lifetime for the interfacial coupling, and $\omega$ the Larmor frequency [25]. The solid line in Fig. 32a is the best fit obtained with Eq. (82), where the leading term is $0.03 \mathrm{~s}^{-1} ; A=10^{7} \mathrm{~s}^{-2}, \tau_{\text {diff }}=71 \mathrm{ps}$, and $\tau_{I}=5 \mu \mathrm{s}$. This logarithmic dependence implies a two-dimensional model, and the much weaker dipolar coupling localizes more strongly the depth of the interfacial region driving the spin relaxation. Thus, a longer correlation time is expected. Assuming that the diffusion constant $D=\frac{l^{2}}{4 \tau}$ and taking $3 \AA$ for the size of the jump length and $\tau_{\text {diff }}=71$ ps gives a translational diffusion of water at the phospholipid interfaces $D=3 \times 10^{-10} \mathrm{~m}^{2} \mathrm{~s}^{-1}$ which is about $1 / 3$ of the bulk water diffusion coefficient.

The observation that the contribution to water proton relaxation rate constants from coupling of water protons to lipid protons is very small, in fact smaller than the water-water proton dipolar contributions in pure water, strongly supports the conclusion that pure lipid interactions make a negligible contribution to water proton spin-lattice relaxation in tissue systems. The relaxation contribution from lipid-based components in tissues is then dominated by the macromolecular assemblies embedded in the lipid, including proteins and carbohydrates.

\subsection{Extreme-values statistics applied to dynamics of water at protein interfaces}

Another area of crucial importance in structural biology is the dynamical behaviour of water at protein interfaces and here too NMRD studies can offer insights. Water dynamics affects the energetic as well as steric aspects of both intra and intermolecular processes, so it is highly desirable to gain a more complete understanding of water molecule dynamics in these complex systems. Important components of the macromolecule and water dynamics occur at relatively low frequencies, on the order of a catalytic rate constant, even though critical motions may also be affected by much faster events. The proton NMRD profile is thus again a powerful approach for characterization of water dynamics. Immobilized proteins present a unique interface with water. Water translational diffusive motions affect not only the high frequency dynamics but also the nuclear spin-lattice relaxation with all surfaces. However, as discussed above, exchange involving rare binding sites for water in protein systems adds very low frequency components to the dynamics spectrum. Water binding sites in protein systems are not identical, thus, distributions of free energies and different dynamics are expected. ${ }^{2} \mathrm{H}_{2} \mathrm{O}$ NMRD profiles characterize the local rotational fluctuations for protein-bound water molecules [38] (Fig. 34). NMRD measurements are sensitive to dynamics down to $10 \mathrm{kHz}$. To account for the data, it has been shown that the extreme-values statistics of rare events [176], i.e. water dynamics in rare binding sites, implies an exponential distribution of activation energies for the strongest binding events [38]. In turn, for an activated dynamical process, this exponential energy distribution leads to a Pareto distribution for the reorientational correlation times, and a power law in the Larmor frequency for the ${ }^{2} \mathrm{H}_{2} \mathrm{O}$ spin-lattice relaxation rate constants at low field strengths [38]. The most strongly held water molecules escape from rare binding sites in times on the order of microseconds, which interrupts the intramolecular correlations and causes a plateau in the spin-lattice relaxation rate at very low frequency. The NMRD data have been analysed by using two simple but related models [38]: a protein-bound environment for water characterized by a single potential well, and a protein-bound environment characterized by a double potential well (Fig. 35) where the potential functions for the local motions of the bound-state water are of different depths. This analysis has been applied to $\mathrm{D}_{2} \mathrm{O}$ deuteriumspin-lattice relaxation on cross-linked albumin and lysozyme, which is dominated by intramolecular relaxation driven by dynamic modulation of the nuclear electric quadrupole coupling [38]. The intramolecular and intermolecular contributions to water-proton spin-lattice relaxation have been separated by isotope dilution, which showed that the intramolecular proton data map onto the deuterium relaxation through a scaling factor that is implied by the relative strength of the quadrupole and dipolar couplings (Fig. 34). The temperature and $\mathrm{pH}$ dependence of the magnetic relaxation dispersion are complex, but may be accounted for by changing only the weighting factors in a superposition of contributions from single-well and double-well contributions (Fig. 35). These experiments show that the reorientational dynamics spectrum for water in and on a protein is characterized by a strongly asymmetric distribution with a long-time tail that extends at least to microseconds.

\subsection{Dimensionality of diffusive exploration of water at the protein interface in solution}

Dynamics of water are critically important to the energies of interaction between proteins and substrates, and hence to the efficiency of transport at the protein interfaces for reaching a catalytic or enzymatic site inside the protein. The NMRD profile of water protons provides a direct characterization of water diffusional dynamics at the protein interface. Values of surface-average translational correlation times are found to be in the range 30-40 ps. The logarithmic NMRD profile measured by Grebenkov et al. [39] is characteristic of 2-dimensional diffusion of water in the protein interfacial region in $\mathrm{H}_{2} \mathrm{O}$ (Fig. 36a) and $\mathrm{D}_{2} \mathrm{O}$ (Fig. 36b). This reduced dimensionality substantially increases the intermolecular reencounter probability and the efficiency of the surface exploration of the water. A comprehensive theory of the translational effects of a small diffusing particle confined in the vicinity of a spherical macromolecule has been proposed as a function of the relative size of the two particles (Fig. 36c) [39]. One finds again a logarithmic frequency dependence of the spectral density for the translational diffusion of water at the surface of spherical protein (Eq. (82)); this calculation is valid provided that the water diffusion remains restricted to a layer that is very thin relative to the protein radius. This shows that the change in apparent dimensionality of diffusive exploration is a general result of the small diffusing particle encountering a much larger particle that presents a diffusion barrier. Examination of the effects of the size of the confinement relative to the size of the macromolecule reveals that the reduced dimensionality characterizing the small molecule diffusion persists down to remarkably small radius ratios. The single-molecule translational correlation is twice the correlation time for the relative interaction or $30 \mathrm{ps}$, which is in good agreement result found from $\mathrm{D}_{2} \mathrm{O}$ (Fig. 36c). Experimental results on several different proteins in solution support this theoretical model, which may be generalized to other small particle-large body systems such as vesicles and micelles. 
9.6. MRI of biological tissues and human brain at variable magnetic fields

Drawing on results from model systems such as those discussed above, one may also interpret results of NMRD experiments with intact biological tissues $[36,177,178]$. This could be of particular importance for understanding the origin of contrast in MRI. The NMRD profile of the composite ${ }^{1} \mathrm{H}_{2} \mathrm{O}$ nuclear magnetic resonance signal $T_{1}$ was measured for excised samples of rat liver, muscle, and kidney over the field range from 0.7 to $7 \mathrm{~T}(35-300 \mathrm{MHz})$ (Fig. 37) with a nuclear magnetic resonance spectrometer using sample-shuttle methods by Diakova et al. [40]. Based on extensive measurements on simpler component systems, the magnetic field dependence of $T_{1}$ of all the tissues studied can readily be fitted at Larmor frequencies above $1 \mathrm{MHz}$ by using a simple relaxation equation consisting of three contributions: a power law related to the interaction of water with long-lived protein binding sites, a logarithmic term related to water diffusion at macromolecular interfacial regions, and a constant term associated with the high frequency limit of water spin-lattice relaxation:

$$
\left.\left.\frac{1}{T_{1}}=A \times \omega^{-0.6}+B \tau_{d} \times\left[\ln 1+\frac{1}{\left(\tau_{d} \times \omega\right)^{2}}\right)+4 \ln 1+\frac{1}{\left(2 \tau_{d} \times \omega\right)^{2}}\right)\right]+C
$$

In Eq. (83) $A$ and $B$ are aggregate constants characterizing the strength of the interactions driving spin relaxation, which are directly related to dipolar couplings, as well as the probabilities for these interactions in various environments. Application of Eq. (83) results in the continuous lines shown in Fig. 37 computed with the parameters summarized in Table 3 . The value of $B$ reflects the probability that the diffusing water molecule is in an interfacial region as well as the distances of closest approach between the water protons and the neighbouring spins that it may sample, including any paramagnetic centers. $\tau_{d}$ is the translational correlation time for water in the interfacial region, which we take as approximately $15 \mathrm{ps}$, a value not very different from that for a lipid interface [39].

The sample-shuttle NMR method turns out to be less applicable in the case of brain tissue because the impact of the shuttle movement affects the integrity of the tissue, but Rooney and collaborators [41] have published data from studies on human volunteers conducted at different field strengths, and used a power law to fit the data well with a small exponent. Interpretation of these has also been carried out using the model described in the previous paragraph, leading to the results summarized in Table 4 [40]. We note that the ratio of the relaxation rate constants reported for the different brain tissues is in a constant ratio to either the grey or white matter values over the whole range of field strengths. As in Fig. 37, the brain tissue data can be normalized or scaled by these factors, leading to the results shown in Fig. 38; the parameters associated with these fits are summarized in Table 4 . The values of $A$ are somewhat smaller than those for muscle and much smaller than those for liver, whereas the values of $B$ are comparable. For white matter structures, in contrast, the value of $B$ is much larger than for grey matter structures, which may reflect differences in the effective interfacial concentration or topography. However, this result may reflect regions of increased translational correlation time or slower diffusion in the interfaces of these tissues than in the others because the coefficient of the logarithmic terms is proportional to the translational correlation time. Direct evidence for the increase in the translational correlation time for the white matter when compared with the grey matter is provided by the steeper slope of the Larmor frequency dependence shown in Fig. 38 for the white matter. This FFC relaxation analysis thus suggests that the water-surface translational correlation time is approximately three times longer than grey matter, in agreement with the diffusion MRI results obtained by Le Bihan [42].

\section{Conclusion}

One and two-dimensional low-field NMR relaxation techniques at fixed and variable frequencies have been described for characterizing the structure and molecular dynamics of various complex liquids embedded in micro-, meso- and macroporous porous media. Several examples were successively presented on calibrated porous silica glasses, granular packings, cement-based, plasters, natural petroleum porous rocks and shale oils. In each case, the nuclear magnetic relaxation dispersion (NMRD) technique has allowed characterization of fundamental properties depending on the nature of the liquid and on their affinity for the pore surface such as surface correlation times, diffusion coefficients and dynamical surface affinity (NMR wettability). Numerous industrial applications of the multiscale NMRD relaxation were presented showing in particular the microstructure evolution (ageing) of various cement-based materials (cement, mortar, and plaster pastes). Other industrial applications reviewed here concerned the extension of the NMRD techniques for separating oil and water in real multimodal macroporous petroleum rocks. For instance, the NMRD, $T_{1}-T_{2}$ and $D-T_{2}$ techniques were presented for separating the dynamics and wettability of oil and water in the organic and mineral dual microporosity of shale oil rocks. Last, examples of these NMRD techniques have shown the complex dynamical spectrum of a folded polymeric structure that span many decades in frequency or time. In contrast, most recent discussions of protein dynamics focus on timescales of nanoseconds and shorter, even though these are far shorter than timescales associated with biological functions; NMRD allows studies of protein dynamics in the frequency range from $10 \mathrm{kHz}$ to $40 \mathrm{MHz}$ or the time range from tens of microseconds to $10 \mathrm{~ns}$. There is still new progress appearing in the field cycling NMR techniques. For instance, dynamic nuclear polarisation (DNP) can enhance the sensitivity of FFC relaxometry at low field [179]. FFC relaxometry with spatial selection has also been proposed for enabling the measurements of dispersion curves from volumes selected from a pilot image [180]. Important new developments have been proposed for extending the NMRD data to very high field (14 T) allowing a better characterization of the contrast agents at relevant magnetic fields for clinical and preclinical MRI [11]. Recently, some FFC proton NMR has been carried out at a ${ }^{1} \mathrm{H}$ frequency down to about $3 \mathrm{~Hz}$ which has allowed exploration of about six decades in frequencies within a single NMRD profile [181]. All the examples shown in this review show that FFC NMR relaxation technique, as well as NMR and ESR spectroscopies, have proven useful for characterizing the multiscale dynamics of complex liquids in bulk and confinement environments.

\section{Acknowledgements}

This work has benefitted from the very fruitful collaboration with my friend Pr. R.G. Bryant (University of Virginia, Charlottesville) in many aspects of NMR in porous media and for all the works reported here on proteins and biological tissues. Many thanks to my colleagues D. Petit (Laboratoire Charles Coulomb (L2C)UMR5221, CNRS-Université de Montpellier), D. Grebenkov, A. LouisJoseph and L. Benamsili (PMC, Ecole Polytechnique) and P. Levitz (Laboratory Phenix, UPMC, Paris) and all the students of the "dream team" at (PMC, Ecole Polytechnique). Special thanks to my partner in the oil industry B. Nicot, (Total EP, Pau) for his very fruitful collaboration in multidimensional NMR experiments in crude and shale oils. Thanks to H.S. Silva, D. Bégué, I. Baraille and B. Bouyssiere 
(IPREM, CNRS-UMR 5254, Pau) for the molecular dynamics simulations of asphaltene aggregation processes. Thanks to I. Jolivet (Total EP) for the FIB-SEM images of shale oil rocks. Many thanks for the interest, stimulation and financial support of I. Betremieux, B. Neff and J.-C. De Witt (Scientific Direction of Total SA of Total, Paris La Défense). Thanks for the very stimulating scientific discussions with P. Ligneul (Schlumberger, Clamart) on phase transitions, spinodal decomposition and Cahn Hilliard equations. Thanks for all the numerous scientific discussions with my new colleagues A. L. Rollet, G. Meriguet and students J.-B. Pigot, A. Fanost and C. Cousin at PHENIX laboratory at UPMC Paris 06, Paris. Last and not least, I would like to thank G. Ferrante, S. Bubicci, M. Pasin and R. Steele from Stelar, Mede, Italy for having facilitated my access to recent Fast-field Cycling NMR spectrometers at Mede.

\section{References}

[1] A.G. Redfield, W. Fite, H.E. Bleich, Precision high speed current regulators for occasionally switched inductive loads, Rev. Sci. Instrum. 39 (1968) 710-715.

[2] R. Kimmich, Field-cycling in NMR relaxation spectroscopy: applications in biological, chemical and polymer physics, Bull. Magn. Reson. 1 (1980) 195217.

[3] F. Noack, NMR field-cycling spectroscopy: principles and applications, Progr. Nucl. Magn. Reson. Spectrosc. 18 (1986) 171.

[4] F. Noack, Basics and novel aspects of NMR field-cycling spectroscopy, Bull. Ampere 175 (1994) 18-35.

[5] R. Kimmich, E. Anoardo, Field-cycling NMR relaxometry, Progr. Nucl. Magn. Reson. Spectrosc. 44 (2004) 257-320.

[6] R. Kimmich, N. Fatkullin, Self-diffusion studies by intra- and inter-molecular spin-lattice relaxometry using field-cycling: liquids, plastic crystals, porous media, and polymer segments, Progr. Nucl. Magn. Reson. Spectrosc. 101 (2017) 18-50.

[7] A.G. Redfield, NMR as a Structural Tool, Plenum, NY, 1996.

[8] K. Victor, V. Kavolius, R.G. Bryant, Magnetic relaxation dispersion probe, J. Magn. Reson. 171 (2004) 253-257.

[9] S. Wagner, T.R.J. Denisen, T. Rayner, R.G. Bryant, High resolution magnetic dispersion measurements of solute spin probes using a dual magnet system, J. Magn. Reson. 140 (1999) 172-178.

[10] A.G. Redfield, High-resolution NMR field-cycling device for full-range relaxation and structural studies of biopolymers on a shared commercial instrument, J. Biomol. NMR 52 (2012) 152-177.

[11] Y. Gossuin, Z. Serhan, L. Sandiford, D. Henrard, T. Marqardsen, R.T.M. Rosales, D. Sakellariou, F. Ferrage, Sample shuttling relaxometry of contrast agents: NMRD profiles above 1 T with a single device, Appl. Magn. Reson. 47 (2016) 237-246.

[12] S.F. Cousin, P. Kaderavek, B. Haddou, C. Charrier, T. Markadsen, J.M. Tyburn, P. A. Bouvier, T. Speck, D. Vilhem, F. Engelke, W. Maas, D. Sakellariou, G. Bodenhausen, P. Pelupessy, F. Ferrage, High-resolution two-field nuclear magnetic resonance spectroscopy, Phys. Chem. Chem. Phys. 18 (2016) 33187-33194.

[13] G. Liu, Y. Li, J. Jonas, Confined geometry-effects on reorientational dynamics of molecular liquids in porous silica glasses, J. Chem. Phys. 95 (1991) 68926901.

[14] J.-P. Korb, S. Xu, J. Jonas, Confinement effects on dipolar relaxation by translational dynamics of liquids in porous silica glasses, J. Chem. Phys. 98 (1993) 2411-2422.

[15] J.-P. Korb, M. Whaley-Hodges, R.G. Bryant, Translational diffusion of liquids at surfaces of microporous materials: theoretical analysis of field cycling magnetic relaxation measurements, Phys. Rev. E 56 (1997) 1934-1935.

[16] J.-P. Korb, M. Whaley-Hodges, T. Gobron, R.G. Bryant, Anomalous surface diffusion of water compared to aprotic liquids in nanopores, Phys. Rev. E 60 (1999) 3097-3106.

[17] S. Stapf, X. Ren, E. Talnishnikh, B. Blümich, Spatial distribution of coke residues in porous catalyst pellets analyzed by field-cycling relaxometry and parameter imaging, Magn. Reson. Imaging 23 (2005) 383-386.

[18] H. Jaffel, J.-P. Korb, J.P. Ndobo-Epoy, V. Morin, J.-P. Guicquero, Probing microstructure evolution during the hardening of gypsum by proton NMR relaxometry, J. Phys. Chem. B 110 (2006) 7385-7391.

[19] H. Jaffel, J.-P. Korb, J.P. Ndobo-Epoy, J.-P. Guicquero, V. Morin, Multi-scale approach continuously relating the microstructure and the macroscopic mechanical properties of plaster pastes during their settings, J. Phys. Chem. B 110 (2006) 18401-18407.

[20] F. Barberon, J.-P. Korb, D. Petit, V. Morin, E. Bermejo, Probing the surface area of a cement-based material by nuclear magnetic relaxation dispersion, Phys. Rev. Lett. 90 (2003) 116103-116107.

[21] A. Plassais, M. Pomies, N. Lequeux, J.-P. Korb, D. Petit, F. Barberon, B. Bresson, Microstructure evolution of hydrated cement pastes, Phys. Rev. E 72 (2005) 041401-041408.

[22] J.-P. Korb, B. Nicot, A. Louis-Joseph, S. Bubici, G. Ferrante, Dynamics and wettability of oil and water in oil shales, J. Phys. Chem. C 118 (2014) 2321223218.
[23] J.-P. Korb, L. Malier, F. Cros, S. Xu, J. Jonas, Surface dynamics of liquids in nanopores, Phys. Rev. Lett. 77 (1996) 2312-2315.

[24] J.Y. Jehng, PhD Thesis, Northwestern University, 1995.

[25] S.G. Godefroy, J.-P. Korb, M. Fleury, R.G. Bryant, Surface nuclear magnetic relaxation and dynamics of water and oil in macroporous media, Phys. Rev. E 64 (2001) 021605-021612.

[26] S.G. Godefroy, M. Fleury, F. Deflandre, J.-P. Korb, Temperature effect on NMR surface relaxation in rocks for well logging applications, J. Phys. Chem. B 106 (2002) 11183-11190.

[27] J.-P. Korb, G. Freiman, B. Nicot, P. Ligneul, Dynamical surface affinity of diphasic liquids as a probe of wettability of multimodal porous media, Phys. Rev. E 80 (2009) 061601-061612.

[28] J.-P. Korb, NMR and nuclear spin relaxation of cement and concrete materials, Curr. Opin. Colloid Interface Sci. 14 (2009) 192-202.

[29] P.J. McDonald, J.-P. Korb, J. Mitchell, L. Monteilhet, Surface relaxation and chemical exchange in hydrating cement pastes: a two-dimensional NMR relaxation study, Phys. Rev. E 72 (2005), 011409-011409.

[30] L. Monteilhet, J.-P. Korb, P.J. Mitchell, P.J. McDonald, Observation of exchange of micropore water in cement pastes by two-dimensional T2-T2 nuclear magnetic resonance relaxometry, Phys. Rev. E 74 (2006) 06404-06409.

[31] K.E. Washburn, P.T. Callaghan, Tracking pore to pore exchange using relaxation exchange spectroscopy, Phys. Rev. Lett. 97 (2006) 17502.

[32] M. Fleury, J. Soualem, Quantitative analysis of diffusional pore coupling from T2-store T2 NMR experiments, J. Colloid Interface Sci. 336 (2009) 250259.

[33] J. Mitchell, M.D. Hürlimann, E.J. Fordham, A rapid measurement of T1/T2: the DECPMG sequence, J. Magn. Reson. 200 (2009) 198-206.

[34] S. Bubici, J.P. Korb, J. Kucerik, P. Conte, Evaluation of the surface affinity of water in three biochars using fast field cycling NMR relaxometry, Magn. Reson. Chem. 54 (2016) 365-370.

[35] K. Victor, J.-P. Korb, R.G. Bryant, Translational dynamics of water at the phospholipid interface, J. Phys. Chem. B 117 (2013) 12475-12478.

[36] S.H. Koenig, R.D. Brown, Field-cycling relaxometry of protein solutions and tissue - implications for MRI, Progr. Nucl. Magn. Reson. Spectrosc. 22 (1990) 487-567.

[37] J.-P. Korb, G. Diakova, Y. Goddard, R.G. Bryant, Relaxation of protons by radicals in rotationally immobilized proteins, J. Magn. Reson. 186 (2007) $176-181$

[38] J.-P. Korb, Y. Goddard, J. Pajski, G. Diakova, R.G. Bryant, Extreme-values statistics and dynamics of water at protein interfaces, J. Phys. Chem. B 115 (2011) 12845-12858.

[39] D. Grebenkov, Y. Goddard, G. Diakova, J.-P. Korb, R.G. Bryant, Dimensionality of diffusive exploration at the protein interface in solution, J. Phys. Chem. B 113 (2009) 13347-13356.

[40] G. Diakova, J.-P. Korb, R.G. Bryant, The magnetic field dependence of water T1 in tissues, Magn. Reson. Med. 68 (2012) 272-277.

[41] W.D. Rooney, G. Johnson, X. Li, E.R. Cohen, S.G. Kim, K. Ugurbil, C.S. Springer Magnetic field and tissues dependencies of human brain longitudinal relaxation in vivo, Magn. Reson. Med. 57 (2007) 308-318.

[42] D.M.L. Bihan, J.-F. Mangin, C. Poupon, S. Pappota, N. Molko, H. Chabriat Diffusion tensor imaging; concepts and applications, J. Magn. Reson. Imaging 13 (2001) 534-546.

[43] A. Abragam, The Principles of Nuclear Magnetism, Clarendon, Oxford, 1961 (Chapter 8).

[44] N. Bloembergen, E.M. Purcell, R.V. Pound, Relaxation effects in nuclear magnetic resonance absorption, Phys. Rev. 73 (1947) 679-712.

[45] P.S. Hubbard, Theory of electron-nucleus Overhauser effects in liquids containg free radicals, Proc. R. Soc. Lond. Ser. 1 (291) (1966) 537.

[46] Y. Ayant, E. Belorisky, J. Alizon, J. Gallice, Calculation of spectral densities for relaxation resulting from random molecular translational modulation of magnetic dipolar coupling in liquids, J. Phys. (France) 36 (1975) 9911004

[47] J.-P. Korb, S. Godefroy, M. Fleury, Surface nuclear magnetic relaxation and dynamics of water and oil in granular packings and rocks, Magn. Reson. Imaging 21 (2003) 193-199.

[48] L.P. Hwang, J.H. Freed, Dynamic effects of pair correlation-functions on spin relaxation by translational diffusion in liquids, J. Chem. Phys. 63 (1975) 4017-4025.

[49] Z. Luz, D. Goldfarb, H. Zimmerman, Nuclear Magnetic Resonance of Liquid Crystals, D. Reidel Publishing Company, Dordrecht, 1985.

[50] M. Brown, J. Seelig, U. Haberlen, Structural dynamics in phospholipid-bilayers from deuterium spin-lattice relaxation-time measurements, J. Chem. Phys. 70 (1979) 5045-5053.

[51] M.F. Brown, Theory of spin-lattice relaxation in lipid bilayers and biologica membranes-H-2 and N-14 quadrupolar relaxation, J. Chem. Phys. 77 (1982) 1576-1599.

[52] M.F. Brown, Unified picture for spin-lattice relaxation of lipid bilayers and biomembranes, J. Chem. Phys. 80 (1984) 2832-2836.

[53] J.W. Emsley, Nuclear Magnetic Resonance of liquid crystals, D. Reidel Publishing Company, Dordrecht, 1985.

[54] J. Seelig, Deuterium magnetic-resonance - theory and application to lipid membranes, Quater. Rev. Biophys. 10 (1977) 353-418.

[55] M.M. Pike, C.S. Springer, Aqueous shift-reagents for high resolution cationic nuclear magnetic resonance, J. Magn. Reson. 46 (1982) 348-353.

[56] R. Kimmich, NMR Tomography, Diffusometry, Springer, Berlin, 1997.

[57] H.M. McConnell, Reaction rates by nuclear magnetic resonance, J. Chem. Phys. 28 (1958) 430-431. 
[58] M. Goldman, T. Tabti, C. Fermon, J.-F. Jacquinot, G. Saux, A model for the influence of motion on the NMR lineshape, J. Magn. Reson. A 103 (1993) 288296.

[59] M. Goldman, A simple formalism for the analysis of NMR in the presence of exchange, Mol. Phys. 86 (1995) 301-315.

[60] F. Cros, L. Malier, J.-P. Korb, F. Chaput, NMR of molecules trapped in sol-gel glasses: progressive closure of the nanoporosity during drying, J. Chim. Phys. Phys.-Chim. Biol. 95 (1998) 264-269.

[61] J. Jeener, Superoperators in magnetic-resonance, Adv. Magn. Reson. 10 (1982) $1-51$.

[62] A.D. Bain, B. Berno, Louivillians in NMR: the direct method revisited, Progr. NMR Spectrosc. 59 (2011) 223-244.

[63] R.B. Lauffer, Paramagnetic metal complexes as water proton relaxation agents for NMR imaging: theory and design, Chem. Rev. 87 (1987) 901-927.

[64] K.R. Browstein, C.E. Tarr, Importance of classical diffusion in NMR-studies of water in biological cells, Phys. Rev. A 19 (1979) 2446-2453.

[65] R.L. Kleinberg, W.E. Kenyon, P.P. Mitra, Mechanism of NMR relaxation of fluids in rock, J. Magn. Reson. A 108 (1994) 206-214.

[66] J.J. Frippiat, M. Letellier, P. Levitz, Interaction of water with clay surfaces, Philos. Trans. R. Soc. Lond. A 311 (1984) 287.

[67] S. Stapf, R. Kimmich, R.O. Seiter, Proton and deuteron field-cycling NMR relaxometry of liquids in porous glasses-evidence for Levy-walk statistics, Phys. Rev. Lett. 75 (1995) 2855-2858.

[68] D.A. Varshalovich, A.N. Moskalev, V.K. Khersonkii, Quantum Theory of Angular Momentum, World Scientific Publishing Co Pte Ltd, Singapore, 1988.

[69] H.S. Carslow, J.C. Jaeger, Conduction of Heat in Solids, Oxford, 1959.

[70] J.-P. Korb, Size effects in the spin-relaxation by bounded diffusion in 2 dimensional systems, J. Chem. Phys. 82 (1985) 1061-1062.

[71] J.-P. Korb, M. Winterhalter, H.M. McConnell, Theory of spin relaxation by translational diffusion in two-dimensional systems, J. Chem. Phys. 80 (1984) 10591068.

[72] J.-P. Korb, Multi-scales nuclear spin-relaxation of liquids in porous media, C. R. Acad. Phys. 11 (2010) 192-203.

[73] I. Solomon, Relaxation processes in a system of 2 spins, Phys. Rev. A 99 (1955) 559-565.

[74] T.A.M.U. Laboratory for Molecular Simulation, Dept of Chem, College of Science, Insight 2, Accelrys, Inc, 1996.

[75] C.C. Lester, R.G. Bryant, Outer coordination sphere-characterization by nuclear magnetic-relaxation dispersion, J. Phys. Chem. B 94 (1990) 2843 2847.

[76] C.F. Polnaszek, R.G. Bryant, Self-diffusion of water at the protein surface - a measurement, J. Am. Chem. Soc. 106 (1984) 428-429.

[77] C.F. Polnaszek, D. Hanggi, P.W. Carr, R.G. Bryant, Nuclear magnetic-relaxation dispersion measurement of water at a silica surface, Anal. Chim. Acta 194 (1987) 311-315.

[78] A. Mitzithras, J.H. Strange, Diffusion of fluids in confined geometry, Magn Reson. Imaging 12 (1994) 261-263.

[79] L. Bocquet, J.L. Barrat, Diffusive motion in confined fluids-Mode-coupling results and molecular dynamics calculations, Europhys. Lett. 31 (1995) 455460.

[80] H. Chemmi, D. Petit, P. Levitz, R. Denoyel, A. Galarneau, J.-P. Korb, Noninvasive experimental evidence of the linear pore size dependence of water diffusion in nanoconfinement, J. Phys. Chem. Lett. 7 (2016) 393-398.

[81] M.C. Bellisent-Funel, S.H. Chen, J.M. Zanotti, Single-particle dynamics of water-molecules in confined space, Phys. Rev. E 51 (1994) 4558-4569.

[82] S.H. Lee, P.J. Rossky, A comparison of the structure and dynamics of liquid water at hydrophobic and hydrophylic surfaces - a molecular-dynamics simulation study, J. Chem. Phys. 100 (1994) 3334-3345.

[83] H.F.W. Taylor, Proposed structure for calcium silicate hydrate gel, J. Amer. Ceram. Soc. 69 (1986) 464-467.

[84] C. Lester, R.G. Bryant, Water-proton nuclear magnetic relaxation in heterogenous systems: hydrated lysozyme results, Magn. Reson. Med. 22 (1991) 143-153.

[85] C. Lester, R.G. Bryant, The magnetic-field dependence of proton spin relaxation in tissues, Magn. Reson. Imaging 22 (1991) 117-126.

[86] J.-P. Korb, P.J. McDonald, L. Monteilhet, A.G. Kalinichev, R.J. Kirkpatrick Comparison of proton field-cycling relaxometry and molecular dynamics simulations for proton-water surface dynamics in cement-based materials, Cem. Concr. Res. 37 (2007) 348-350.

[87] J.-P. Korb, L. Monteilhet, P.J. McDonald, J. Mitchell, Microstructure and texture of hydrated cement-based materials: a proton field cycling relaxometry approach, Cem. Concr. Res. 37 (2007) 295-302.

[88] J.J. Thomas, H.M. Jennings, A.J. Allen, Analysis of CSH gel and cement paste by small-angle-neutron scattering, Concr. SCI Eng. 1 (1999) 45-64.

[89] R.L. Rarick, J.J. Thomas, B.J. Christensen, H.M. Jennings, Deterioration of the nitrogen BET surface area of dried cement paste with storage time, Adv. Cem. Based Mater. 3 (1996) 72-75.

[90] E. Sauzeat, Thesis, Institut National de Lorraine, Nancy, 1998

[91] D.N. Winslow, S. Diamond, Specific surface of hardened Portland-cement paste as determined by small-angle X-ray-scattering, J. Am. Chem. Soc. 57 (1974) 193-197.

[92] A.J. Allen, Time-resolved phenomena in cements, clays and porous rocks, J. Appl. Cryst. 24 (1991) 624-634.

[93] W. Halperin, J. Jehng, Y. Song, Application of spin-spin relaxation to measurement of surface-area and pore-size distributions in a hydrating cement paste, Magn. Reson. Imaging 12 (1994) 169-173.
[94] R. Maggion, Thesis, University of Orléans, 1992.

[95] L. Barbic, I. Kocuvan, R. Blinc, The determination of surface development in cement pastes by nuclear magnetic-resonance, J. Am. Chem. Soc. 65 (1982) 25-31.

[96] N. Nestle, A simple semi-empiric model for NMR relaxometry data of hydrating cement pastes, Cem. Concr. Res. 34 (2002) 447-454.

[97] R.M.E. Valckenborg, L. Pel, K. Kopinga, Combined NMR cryoporometry and relaxometry, J. Phys. D 35 (2002) 249-253.

[98] F. Sorrentino, R. Castanet, Application of thermal analysis to the cement industry, J. Therm. Anal. 38 (1992) 2147-2155.

[99] G.E. Pake, Nuclear resonance absorption in hydrated crystals - fine structure of the proton line, J. Chem. Phys. 16 (1948) 327-336.

[100] K.M. Song, J. Mitchell, H. Jaffel, L.F. Gladden, Simultaneous monitoring of hydration kinetics, microstructural evolution, and surface interactions in hydrating gypsum plaster in the presence of additives, J. Mater. Sci. 45 (2010) 5282-5290.

[101] H. Jaffel, Caractérisation multi-échelles de matériaux poreux en évolution; cas du plâtre, Physics Department, Ecole Polytechnique, 2006, Thesis.

[102] J.-P. Korb, P. Levitz, Direct probing of the wettability of plaster pastes at the nanoscale by proton field cycling relaxometry, in: AIP Conference Proc., 2008, pp. 55-58.

[103] H. Lechatelier, C. R. Acad. Sci. 96 (1883) 715.

[104] P.E. Levitz, Random flights in confining interfacial systems, J. Phys. Condens. Matter. 17 (2005) S4059-S4074.

[105] P.E. Levitz, J.-P. Korb, Probing glass transition of clay colloids by NMR relaxometry: interplay between fluid Brownian dynamics and particle jamming, Europhys. Lett. 70 (2005) 684-689.

[106] C.D. Pasquale, V. Marsala, A.E. Berns, M. Valagusa, A. Pozzi, G. Alonzo, Fastfield cycling NMR relaxometry characterization of biochars obtained from an industrial thermochemical process, J. Sol. Sediments 12 (2012) 1211-1221.

[107] A. Gaspar, E. Zellermann, S. Lababidi, J. Reece, W. Schrader, Characterization of saturates, aromatics, resins and asphaltenes heavy crude oil fractions by atmospheric pressure laser ionization Fourier transform ion cyclotron resonance mass spectroscopy, Energy Fuels 26 (2012) 3481-3487.

[108] O.C. Mullins, The asphaltenes, Annu. Rev. Anal. Chem. (Palo Alto Calif) 4 (2011) 393-418.

[109] J. Eyssautier, P. Levitz, D. Espinat, J. Jestin, J. Gummel, J. Grillo, L. Barré, Insight into asphaltene nanoaggregate structure inferred by small angle nautron and X-ray scattering, J. Phys. Chem. B 115 (2011) 6827-6837.

[110] N. Vorapalawut, B. Nicot, A.L. Louis-Joseph, J.-P. Korb, Probing dynamics and interaction of maltenes with asphaltenes aggregates in crude oils by multiscale NMR, Energy Fuels 29 (2015) 4911-4920.

[111] C.L.B. Guedes, E.D. Mauro, V. Antunes, A.S. Mangrich, Photochemical weathering study of Brazilean petroleum by EPR spectroscopy, Mar. Chem. 84 (2003) 105-112.

[112] L. Benamsili, J.-P. Korb, G. Hamon, A. Louis-Joseph, B. Bouyssière, H. Zhou, R.G. Bryant, Multi-dimensional magnetic resonance characterization of dynamics and saturations of brine/crude oil/mud filtrate mixtures confined in rocks, Energy Fuels 28 (2014) 1629-1640.

[113] G. Hirasaki, S.W. Lo, Y. Zhang, NMR properties of petroleum fluids, Magn. Reson. Imaging 21 (2003) 269-277.

[114] J.-P. Korb, N. Voralpalawut, B. Nicot, R.G. Bryant, Relation and correlation between NMR relaxation times, diffusion coefficients and viscosity in heavy crude oils, J. Phys. Chem. C 119 (2015) 24439-24446.

[115] O.C. Mullins, D.J. Seiffert, J.Y. Zuo, M. Zeybeck, Nanoaggregates observed in oilfield reservoirs, Energy Fuels 27 (2013) 1752-1761.

[116] R. Rodgers, S. Rowland, D. Smith, G. Blakney, M. Chacon, J. Putman, A Mckenna, Y. Corilo, D. Podgorski, P. Lalli, A. Clingenpeel, J. Lu, A. Marshall, C. Hendrisckson, Contributions of high resolution mass spectrometry to the field of petroleomics, in: P. Giusti, B. Bouyssiere, H. Carrier, C. Alfonso (Eds.), Petrophase 2017, Le Havre, June 11-15, 2017.

[117] B. Schuler, G. Meyer, D. Pena, O.C. Mullins, L. Gross, Unraveling the molecular structures of asphaltenes by atomic force microscopy, J. Am. Chem. Soc. 137 (2015) 9870-9876.

[118] A.C.R. Sodero, H.S. Silva, P. Guevara, B. Bouyssiere, J.-P. Korb, H. Carrier, A. Alfara, D. Bégué, I. Baraille, Investigation of the effect of sulfur heteroatom on asphaltene aggregation, Energy Fuels 30 (2016) 4758-4766.

[119] H.S. Silva, A.C.R. Sodero, B. Bouyssiere, H. Carrier, J.-P. Korb, A. Alfara, C. Vallverdu, D. Bégué, I. Baraille, Molecular dynamics study of nanoaggregation in asphaltene mixtures: effects of the N, O, and S heteroatoms, Energy Fuels 30 (2016) 5656-5664.

[120] P. Singer, Z. Chen, L.B. Alemany, G.J. Hirasaki, NMR relaxation of polymeralkane mixes, a model systems for crude oils, SPWLA, in: 58th Ann. Log. Symposium, 2017, pp. 1-18.

[121] H.S. Silva, A.C.R. Sodero, J.-P. Korb, A. Alfara, P. Giusti, G. Vallverdu, D. Bégué, I. Baraille, B. Bouyssiere, The role of metalloporohyrins on the physicalchemical properties of petroleum fluids, Fuel 188 (2017) 374-381.

[122] M. Alizadehgiashi, J.M. Shaw, Fickian and non-Fickian diffusion in heavy oil + light hydroacarbon mixtures, Energy Fuels 29 (2015) 2177-2189.

[123] J.W. Cahn, J.E. Hilliard, Free energy of a nonuniform system. I. Interfacial energy, J. Chem. Phys. 28 (1958) 258.

[124] M.D. Hürliman, M. Flaum, L. Venkataramanan, C. Flaum, R. Freedman, G.J. Hirasaki, Diffusion-relaxation distribution functions of sedimentary rocks in different saturation states, Magn. Reson. Imaging 21 (2003) 305-310.

[125] L. Benamsili, J.-P. Korb, G. Hamon, A. Louis-Joseph, B. Bouyssière, H. Zhou, R.G. Bryant, Multi-dimensional magnetic resonance characterizations of 
dynamics and saturations of brine/crude oil/mud filtrate mixtures confined in rocks: the role of asphaltene, Energy Fuels 28 (2014) 1629-1640.

[126] M.D. Hürlimann, M. Flaum, L. Venkataramanan, C. Flaum, R. Freedman, G. Hiraski, Diffusion-relaxation distributions functions of sedimentary rocks in different saturation states, Magn. Reson. Imaging 21 (2003) 305-310.

[127] B. Nicot, Détermination de la viscosité des pétroles bruts lourds par relaxation RMN, Université Pierre et Marie Curie, Paris, 2007, Thesis.

[128] A.R. Mutina, M.D. Hürlimann, Correlation of transverse and rotational diffusion coefficient: a probe of chemical composition in hydrocarbon oils, J. Phys. Chem. A 112 (2008) 3291-3301.

[129] J.J. Chen, M. Hürlimann, J. Paulsen, D. Freed, S. Mandal, Y.-Q. Song, Dispersion of T1 and T2 NMR relaxation in crude oils, Chemphyschem Com. 15 (2014) 2671-2681.

[130] E.C. Donaldson, R.D. Thomas, P.B. Lorenz, Wettability determination and its effect on recovery efficiency, Soc. Petrol. Eng. J. 9 (1969) 13.

[131] W.G. Anderson, Wettability literature survey. 2. Wettability measurement, J. Petrol. Technol. 38 (1986) 1125-1144.

[132] M. Robin, Interfacial phenomena: reservoir wettability in oil recovery, Oil Gas Sci. Technol. Rev. D IFP Energies Nouvelles 56 (2001) 55-62.

[133] E. Amott, Observation relating to the wettability of porous rock, Trans. AIME Min. Metal. Eng. 216 (1959) 156-162.

[134] W.J. Looyestijn, Wettability index determination from NMR logs, Petroph. 49 (2008) 130-145.

[135] M. Fleury, F. Deflandre, Quantitative evaluation of porous media wettability using NMR relaxometry, Magn. Reson. Imaging 21 (2003) 385-387.

[136] J.-P. Korb, A. Louis-Joseph, L. Benamsili, Probing structure and dynamics of bulk and confined crude oils by multiscale NMR spectroscopy, diffusometry and relaxometry, J. Phys. Chem. B 117 (2013) 7002-7014.

[137] C.L.B. Guedes, E.D. MAuro, V. Antunes, A.S. Mangrich, Photochemical weathering study of Brasilean petroleum by EPR spectroscopy, Mar. Chem. 84 (2003) 105-112.

[138] O. Delpoux, J. Barbier, J. Marques, J. Verstraete, H. Vezin, Pulsed ESR and porphyrine, in: Proceedings of the 14th International Conference on Petroleum Phase Behavior and Fouling, Petrophase, Poster PB33 (RueilMalmaison, France, 10-13 June 2013), 2013.

[139] S. Stapf, A. Ordikhani-Seyedlar, N. Ryan, C. Mattea, R. Kausic, D. Freed, Y.Q. Song, M. Hürlimann, Probing maltene-asphaltene interaction in crude oil by means of NMR relaxation, Energy Fuels 28 (2014) 2395-2401.

[140] J.-P. Korb, B. Nicot, I. Jolivet, Dynamics and wettability of petroleum fluids in shale oil probed by 2D T1-T2 and fast field cycling NMR relaxation, Microporous Mesoporous Mater. (in press, corrected proof, available on line 31 May 2017 (2017)) https://doi.org/10.1016/j.micromeso.2017.05.055.

[141] A. Valori, Characterization of Cementitious Materials by ${ }^{1} \mathrm{H}$ NMR, Dept of Physics, University of Surrey, 2009, Thesis.

[142] M. Fleury, M.F.F. Romero-Sarmiento, Characterization of shales using T1-T2 NMR maps, J. Petrol. Sci. Eng. 137 (2016) 55-62.

[143] Z. Chen, P.M. Singer, G. Hirasaki, Effects of bitumen extraction on the 2D NMR response of saturated kerogen isolates, SPWLA, in: 58th Ann. Logging Symp., 2017

[144] B. Nicot, N. Vorapalawut, B. Rousseau, L.F. Madariaga, G. Hamon, J.-P. Korb, Estimating saturations in organic shales using 2D NMR SCA A024, in: International Symposium of the Society of Core Analysts, St. John's Newfoundland and Labrador, Canada, 2015.

[145] F. Dalas, J.-P. Korb, S. Pourcher, A. Nonat, D. Rinaldi, Surface relaxivity of cement hydrates, J. Phys. Chem. C 118 (2014) 8387-8396.

[146] M.E. Curtiss, R.J. Ambrose, C.H. Sondergeld, C.S. Rai, Structural characterization of gas shales on the micro- and nano-scales, in: Canadian Unconventional Resources \& International Petroleum Conference, Alberta, Canada, Calgary, 2010, p. 137693.

[147] R.M. Daniel, R.V. Dunn, J.L. Finney, J.C. Smith, The role of dynamics in enzyme activity, Annu. Rev. Biophys. Biomol. Struct. 32 (2003) 69-92.

[148] H. Frauenfelder, B.H. McMahon, Dynamics and function of proteins: the search for general concepts, Proc. Natl. Acad. Sci. U.S.A. 95 (1998) 47954797.

[149] H. Frauenfelder, B.H. McMahon, R.H. Austin, K. Chu, J.T. Groves, The role of structure, energy landscape, dynamics, and allostery in the enzymatic function of myoglobin, Proc. Natl. Acad. Sci. U.S.A. 98 (2001) 2370-2374.

[150] V. Reat, H. Patzelt, M. Ferrand, C. Pfister, D. Oesterhelt, G. Zaccai, Dynamics of different functional parts of bacteriorhodopsin $\mathrm{H}-2 \mathrm{H}$ labeling and neutron scattering, Proc. Natl. Acad. Sci. U.S.A. 95 (1998) 4970-4975.

[151] J.A. Wand, Dynamic activation of protein function: a view emerging from NMR spectroscopy, Natl. Struct. Biol. 8 (2001) 926-931.

[152] B. Brooks, M. Karplus, Harmonic dynamics of proteins: normal modes and fluctuations in bovine pancreatic trypsin inhibitor, Proc. Natl. Acad. Sci. U.S.A. 80 (1983) 6571-6575.

[153] N. Go, T. Noguti, T. Nishikawa, Dynamics in small globular proteins in terms of low-frequency vibrational modes, Proc. Natl. Acad. Sci. U.S.A. 80 (1983) $3696-3700$

[154] J.-P. Korb, R.G. Bryant, The physical basis for the magnetic field dependence of protonspin-lattice relaxation rates in proteins, J. Chem. Phys. 115 (2001) 10964-10974.

[155] J.-P. Korb, R.G. Bryant, Noise and functional protein dynamics, Biophys. J. 89 (2005) 2685-2692.

[156] D.M. Korzhnev, X. Salvatella, M. Vendruscolo, A.A.D. Nardo, A.R. Davidson, L.E. Kay, Low-populated folding intermediates of Fyn SH3 characterized by relaxation dispersion NMR, Nature 430 (2004) 586-590.
[157] B. Halle, V.P. Denisov, K. Venu, Multinuclear relaxation dispersion studies of protein hydration, in: N.R. Krishna, L.J. Berliner (Eds.), Biological Magnetic Resonance, Kluwer/Plenum, New York, 1999.

[158] W.M. Shirley, R.G. Bryant, Proton-nuclear spin-relaxation and molecular dynamics in the lysozyme-water system, J. Am. Chem. Soc. 104 (1982) 2910 2918.

[159] T. Miyazawa, T. Shimanouchi, S. Mizushima, Normal vibrations of N-methy acetamide, J. Chem. Phys. 29 (1958) 611-616.

[160] S. Alexander, R. Orbach, Density of states on fractals-Fractons, J. Phys. (France) Lett. 43 (1982) L625-L631.

[161] S. ALexander, Vibrations of fractals and scattering of light from aerogels, Phys. Rev. B 40 (1989) 7953-7965.

[162] G. Diakova, Y. Goddard, J.-P. Korb, R.G. Bryant, Water and backbone dynamics in a hydrated protein, Biophys. J. 98 (2010) 138-146.

[163] R. Kimmich, F. Winter, Double-diffusive fluctuations and the $f^{\wedge}(3 / 4)$ law of proton spin-lattice relaxation in bioploymers, Progr. Colloid Polym. Sci. 71 (1985) 66-70.

[164] J.-P. Korb, G. Diakova, R.G. Bryant, Paramagnetic relaxation of protons in rotationally immobilized proteins, J. Chem. Phys. 124 (2006) 134910 134916.

[165] N. Bloembergen, L.O. Morgan, Proton relaxation times in paramagnetic solutions effects of electron spin relaxation, J. Chem. Phys. 34 (1961) 842 850.

[166] I. Bertini, J. Kowalewski, C. Luchinat, R. Nilsson, G. Parigi, Nuclear spin relaxation in paramagnetic complexes of $\mathrm{S}=1$ : electron spin relaxation effetcs, J. Chem. Phys. 111 (1999) 5795-5807.

[167] D. Kruk, J. Kowalewski, Nuclear spin relaxation in paramagnetic systems ( $\mathrm{S}>=1$ ) under fast rotation conditions, J. Magn. Reson. 162 (2003) 229-240.

[168] P.H. Fries, G. Ferrante, E. Belorizki, S. Rast, The rotational motion an electronic relaxation of the $\mathrm{Gd}(\mathrm{III})$ aqua complex in water revisited through full proton relaxivity study of a probe solute, J. Chem. Phys. 119 (2003) 86368644.

169] M.W. Hodges, D.S. Cafiso, C.F. Polnaszek, C.C. Lester, R.G. Bryant, Wate translational motion at the bilayer interface: an NMR relaxation dispersion measurement, Biophys. J. 73 (1997).

[170] J. Crank, The Mathematics of Diffusion, second ed., Clarendon, Oxford, 1975.

[171] D.S. Grebenkov, NMR survey of reflected Brownian motion, Rev. Mod. Phys. 79 (2007) 1077-1137.

[172] R. Kimmich, G. Voigt, Nuclear magnetic relaxation dispersion in lecithin bilayers, Chem. Phys. Lett. 62 (1979) 181-183.

[173] R. Eberhard, F. Noack, Proton spin relaxation dispersioin studies of phospholipid membranes, J. Phys. Chem. 92 (1988) 2981-2987.

[174] C.C. Lester, R.G. Bryant, Magnetically coupled paramagnetic relaxation agents, Magn. Reson. Imaging 24 (1992) 236-242.

[175] J.-P. Korb, A. Delville, S. Xu, G. Demeulenaere, G. Costa, Relative role of surface interactions and topological effects in nuclear-magnetic-resonance of confined liquids, J. Chem. Phys. 101 (1994) 7074-7081.

[176] E. Gumbel, Statistics of Extremes, Columbia University Press, New York, 1958

[177] R.G. Bryant, D. Mendelson, C.C. Lester, The magnetic field dependence o proton spin relaxation in tissues, Magn. Reson. Med. 199 (1991) 117-126.

[178] S.H. Koenig, Molecular basis of magnetic relaxation of water protons of tissue, Acad. Radiol. 3 (1996) 597-606.

[179] O. Neudert, H.-P. Raich, C. Mattea, S. Stapf, K. Münnemann, An AldermanGrant resonator for S-band dynamic nuclear polarization, J. Magn. Reson. 242 (2014) 79-85.

[180] K.J. Pine, G.R. Davies, D.J. Lurie, Field-cycling NMR relaxometry with spatia resolution, Magn. Reson. Med. 63 (2010) 1698-1702.

[181] B. Kresse, M. Becher, A.F. Privalov, M. Hofmann, M. Vogel, F. Fujara, E.A Rössler, Proton NMR at Larmor frequencies down to $3 \mathrm{~Hz}$ by means of fieldcycling techniques, in: 10th Conference on Fast Field Cycling NMR Relaxometry, Mikolajki, Poland, 2017.

\section{Glossary}

A: Surface affinity parameter (NMR wettability) $A=\tau_{\mathrm{s}} / \tau_{\mathrm{m}}$

$B_{0}$ : Constant magnetic field

$B_{\mathrm{p}}$ : Polarization field

BPP: Bloembergen-Purcell-Pound

C8: Chain length of octane

CA: Contact angle

$c_{\text {asph }}$ : Asphaltene concentration (\%wt)

CPMG: Carr-Purcell-Meiboom-Gill (NMR sequence for measuring $\mathrm{T}_{2}$ )

CSH: Tricalcium silicate hydrate of cement

$D$ : Translational diffusion coefficient

$D_{1 \mathrm{D}}$ : 1-Dimensional translational diffusion coefficient

$\langle d\rangle$ : Average distance between asphaltene macro-aggregates

$d_{\mathrm{f}}$ : Mass fractal dimension

$d_{\text {pore: }}$ Average pore diameter

$d_{s}$ : Spectral dimension: i.e. the dimension of random walk in a fractal network

$D_{\text {surf: }}$ Surface translational diffusion coefficient

1D: One-dimensional

2D: Two-dimensional

$D-T_{2}: 2 \mathrm{D}$ spin-correlation map between $\mathrm{D}$ and $\mathrm{T}_{2}$

ESR: Electronic spin resonance 
F: Ratio of the solid-proton magnetization to the liquid-proton magnetization at equilibrium

$\mathrm{Fe}^{3+}$ : Ferric ion

FFC: Fast-Field Cycling relaxometry

FIB: Focused ion beam

$G(\ldots)$ : Correlation function

${ }^{1} \mathrm{H}$ : Proton

${ }^{2} \mathrm{H}$ : Deuterium

$h$ : Planck constant

$h$ : Planck constant $\mathrm{h} / 2 \pi$

I: Quantum number of a nuclear spin

$J(\ldots)$ : Spectral density

$k_{\mathrm{B}}$ : Boltzmann factor

$\mathrm{Mn}^{2+}$ : Manganese II ion

$M_{\mathrm{z}}(\ldots)$ : Longitudinal magnetization

$N$ : Number of protons

NMR: Nuclear magnetic resonance

NMRD: Nuclear magnetic relaxation dispersion

$N P$ : Non-prepolarized FFC sequence

PP: Pre-polarized FFC sequence

ppm: Part per million

$R_{1}$ : Longitudinal relaxation rate $=1 / \mathrm{T}_{1}$

$R_{1, \text { bulk: }}$ Bulk longitudinal relaxation rate

$R_{1, \text { surface: }}$ Longitudinal surface relaxation rate

$R_{2}$ : Transverse relaxation rate $=1 / \mathrm{T}_{2}$

$R_{\mathrm{agg}}$ : Radius of asphaltene macro-aggregate

$R_{\mathrm{mol}}$ : Radius of maltene molecule

$S / V$ : Surface to volume ratio

SANS: Small angle neutron scattering

SAXS: Small angle X-ray scattering

SEM: Scanning electron microscopy

$S_{\mathrm{p}}$ : True specific surface area

$S_{P, N M R}:$ NMR specific surface area $=S_{p} F$

STEM: Scanning transmission electron microscopy

$S_{\mathrm{w}}$ : Water saturation of a rock core

$T$ : Absolute temperature

$t$ : Time

$T_{1}$ : Spin-lattice relaxation time
$T_{1, \mathrm{Fe}}$ : Electronic spin-lattice time of the paramagnetic $\mathrm{Fe}^{3+}$ ions

$T_{1, \text { param }}$ : Longitudinal paramagnetic relaxation time

$\left\langle T_{1, \mathrm{LM}}\right\rangle$ : Logarithmic average longitudinal relaxation time

$\left\langle T_{2, \mathrm{LM}}\right\rangle$ : Logarithmic average transverse relaxation time

$T_{1}-T_{2}: 2 \mathrm{D}$ spin-correlation map between $\mathrm{T}_{1}$ and $\mathrm{T}_{2}$

$T_{2}$ : Transverse relaxation time

USBM: U.S. Bureau of Mines (Industrial wettability index used in the oil industry)

$V$ : Pore volume

$V / V_{0}$ : Filling factor of the pore

$\mathrm{VO}^{2+}$ : Paramagnetic vanadyl ion

$w / c$ : Water to cement ratio

$w / p$ : Water to plaster ratio

$\Phi$ : Porosity

TEM: Transmission electron microscopy

$\delta_{\mathrm{IS}}$ : Distance of minimal approach between nuclear I and electronic S spins

$\delta_{\text {oil }}:$ Average molecular size of a hydrocarbon molecule

$\varepsilon:$ Water molecule size $\sim 3 \AA$

$\eta$ : Viscosity

$\eta_{\mathrm{c}}$ : Threshold viscosity

$\lambda$ : Height of liquid surface layer at a pore surface

$v_{0}$ : Larmor frequency

$\rho_{1,2}$ : Longitudinal and surface relaxivity

$\rho_{\text {Gyp }}$ : Density of Gypsum

$\rho_{\text {oil }}:$ Oil density

$\rho_{\text {Shale: }}$ Density of shale rock

$\sigma_{\mathrm{s}}$ : Surface density of paramagnetic species

$\tau$ : Time of evolution

$\tau_{1 \mathrm{D}}$ : 1D translation correlation time

$\tau_{\mathrm{A}}$ : Adsorption correlation time on surface

$\tau_{\text {ex }}$ : Correlation time for exchange

$\tau_{\mathrm{m}}$ : Surface correlation time for translational diffusion of liquid

$\tau_{\text {rot }}:$ Rotational correlation time

$\tau_{\mathrm{s}}$ : Time of residence of embedded liquid at a pore surface

$\omega_{\mathrm{A}}$ : Characteristic frequency for surface adsorption

$\omega_{\mathrm{s}}$ : Electronic Larmor precession frequency

$\omega_{0}$ : Nuclear Larmor precession frequency $=2 \pi v_{0}$

$\omega_{\mathrm{d}}$ : Intermolecular dipolar frequency ${ }^{*} 2 \pi$ 